\title{
IV. On the effect of stress on magnetization and its reciprocal relations to the change of elastic constants by magnetization
}

\section{K. Honda \& T. Terada}

To cite this article: K. Honda \& T. Terada (1907) IV. On the effect of stress on magnetization and its reciprocal relations to the change of elastic constants by magnetization, Philosophical Magazine Series 6, 14:79, 65-115, DOI: 10.1080/14786440709463662

To link to this article: http://dx.doi.org/10.1080/14786440709463662

曲 Published online: 16 Apr 2009.

Submit your article to this journal $[\pi$

Џll Article views: 7

Q View related articles $\longleftarrow$

Citing articles: 3 View citing articles $\square$ 
the series of holes (considered as a grating) is in the act of passing off, and it is evident that the peculiar interference is due to this circumstance *. The argument applies even more strongly, if less simply, to an actual grating formed by a series of narrow parallel and equidistant slits cut in an infinite screen.

The case of a reflecting grating differs in some important respects from that above considered. An investigation applicable to light is now nearly completed. It confirms the general conclusion that peculiarities are to be looked for at such angles of incidence that spectra of higher order are just passing off, but (it is especially to be noted) only when the polarization is such that the electric vector is perpendicular to the grating.

Terling. Place, Witham, May 4.

P.S. June 5.-In answer to further inquiry Prof. Wood tells me that he thinks the ruling may perhaps be 15020 to the inch, but (the grating being for the time out of his hands) he is not able to speak with certainty.

IV. On the Effect of Stress on Magnetization and its Reciprocal Relations to the Change of "Elastic Constants by Magnetization. By K. Honda and T. Terada, Lecturers on Physics in the Tökyō Imperial University $\dagger$.

[Plates I.-V.]

TN our previous experiments, we investigated in some 1 detail the change of elastic constants of several ferromagnetic metals and alloys caused by magnetization, with special regard to the order of applying the stress and the field, and found that in some cases the change is considerably large, and moreover that it differs more or less for different orders of applying the stress and the field. In order to find the proper explanation of these facts, it will be necessary to investigate with the same specimens as in the previous experiment the change of magnetization by stress, with

* If $\epsilon$ be not a precise multiple of $\lambda$, the series $1+\frac{1}{2}+\frac{1}{3}+\ldots$. ad infin. would be replaced by

which is equivalent to

$$
e^{-i \epsilon_{\epsilon}}+\frac{1}{2} e-2 i \epsilon_{\epsilon}+\frac{1}{3} e^{-3 i k_{\epsilon}}+\cdots \cdots
$$

$$
-\log \left\{2 \sin \left(\frac{1}{2} k \epsilon\right)\right\}+\frac{1}{2} i(k \epsilon-\pi) \text {. }
$$

+ From the Journ. Coll. Sci. Tokyo. Communicated by the Authors. Phil. Mag. S. 6. Vol. 14. No. 79. July 1907, 
special regard to the order of applying the stress and the field.

Since J.J. Thomson * gave his theoretical exposition of the reciprocal relations between magnetism and strain, several theories $\dagger$ in the same field have been published, and the present investigation may also afford interesting materials for testing the validity of these theories. In this direction, we have been preceded by Rensing $\ddagger$ and Cantone $\$$, in the case of iron and nickel; but a more extended research may not be undesirable. With this view, sets of experiments have been undertaken, firstly to investigate the change of magnetization by applying successive stresses under constant fields; and secondly, to investigate the magnetization by applying the magnetizing field under different constant stresses and thence to deduce indirectly the change of magnetization by stresses.

Specimens used had the following dimensions :-

\begin{tabular}{|l|l|l|c|}
\hline \multicolumn{1}{|c|}{ Specinens. } & Length. & Dianeter. & $\begin{array}{c}\text { Demagnetizing } \\
\text { factor. }\end{array}$ \\
\hline Swedish iron. & $21.80 \mathrm{~cm}$. & $0.903 \mathrm{~mm}$. & 0.00089 \\
Tungsten steel. & 26.85 & 0.885 & 0.00050 \\
Nickel. & 26.87 & 0.863 & 000056 \\
28.74 per cent. Ni. & 26.80 & 0.964 & 0.00063 \\
50.72 per cent. Ni. & 27.00 & 0.880 & 0.00050 \\
70.32 per cent. Ni. & 26.86 & 0.891 & 0.00068 \\
\hline
\end{tabular}

\section{\$1. Apparatus.}

The intensity of magnetization was measured by the ballistic method. This method was preferred to the magnetometric one, since in our experiments fields up to 400 c.G.s. units were required, and consequently the adjustment of a very sensitive magnetometer placed close to a pair of compensating coils traversed by a strong current would be extremely troublesome. The magnetizing coil, which was

* J. J. Thomson, 'Application of Dynamics to Physies and Chemistry, Chap. iv.

† F. Kolácêk, Ann. d. Phys xiii. p. 1 (1904); Ann. d. Phys. xiv, p. 177 (1904). A. Heydweiller; Ann. d. Phys. xi. p. 602 (1903). R. Gans, Ann. d. Phys. xiij. p. 634 (1904). S. Sano, Proc. Tokyo Math.Phys. Soc. ii. p. 175 and p. 207 (1904).

\pm Rensing, Ann. d. Phys. xiv. p. 363 (1904).

\$ Cartone, Rend. d. Ist. Lomb. (2) xxxvii. p. 435 (1904). Ibid. pp. $474,535 \& 567$. 
one that had been used in our previous experiments, was placed in its vertical position. The length and the constant of the coil were respectively $40 \mathrm{~cm}$. and $392 \cdot 6$. A secondary coil was wound on a glass tube (external diameter $1.5 \mathrm{~cm}$. ), consisting of 1246 turns of well insulated copper wire (diameter $=0.56 \mathrm{~mm}$.) in six layers, the length of the coil being $14 \mathrm{~cm}$. This secondary coil was fixed coaxially in the magnetizing coil, so that the former might lie in a uniform field excited by the latter.

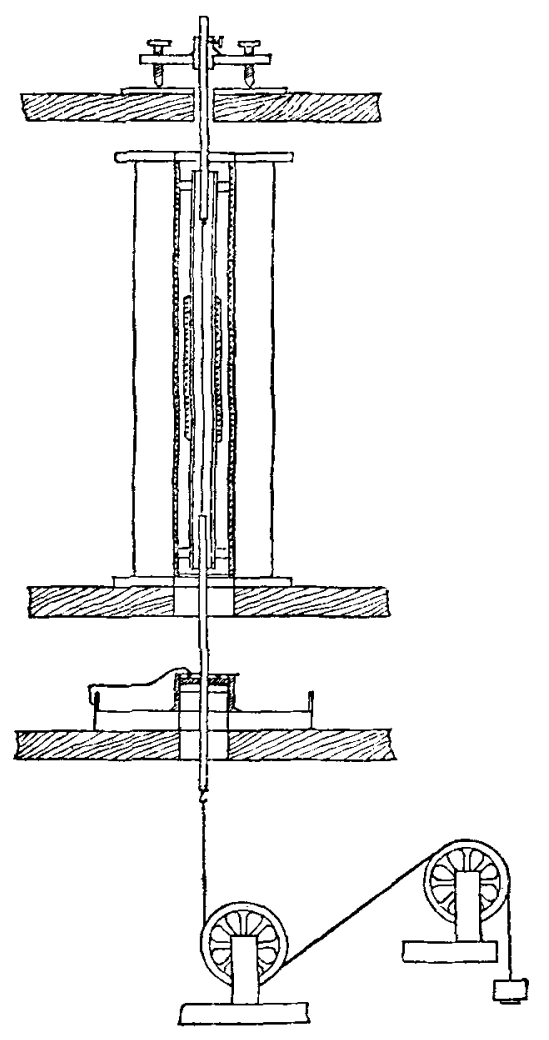

To compensate for the induction due to the magnetizing field alone, a similar secondary coil connected in series with the above secondary coil was inserted within another coil equal to and connected in series with the magnetizing coil, so that by sliding the secondary within the primary, the induction could be compensated to any desired degree. These two pairs of coils were placed at a sufficient distance from each other to prevent their mutual action. The ballistic galvanometer for measuring the induced current due to the magnetization of the specimen was drum shaped, with $0.8 \Omega$ resistance ; a mirror with a small magnet was suspended in the centre of the coil by a spider tbread. Its period

of oscillation was about 9 seconds. The galvanometer was connected with the secondary circuit of the system and placed at a distance of about 15 metres from the magnetizing coil to avoid its direct action. The galvanometer was, however, still disturbed when a strong current was switched on to the magnetizing coil. To prevent this, the compensating primary 
was so directed that the direct effect of the combined system on the galvanometer was null. To determine the constant of the galvanometer, we should have used the compensating secondary coil, if it had been wound in one layer, so that its effective sectional area could be determined with sufficient accuracy. But, as the ambiguity of the sectional area in the secondary coil of 6 layers was inevitable, another coil was constructed with a thin copper wire wound on a wooden cylinder of $5.04 \mathrm{~cm}$. diameter, in a single layer; the number of turns of the coil was 48 . This was always put in series with the secondary circuit and placed at a sufficient distance to be safe from any sensible influence of the magnetizing circuit during the experiments for magnetization. When the constant of the galvanometer was to be determined, the compensating secondary coil was removed from the primary coil and replaced by this coil; then the magnetizing coil for the specimen was shunted off, a weak magnetizing current of known strength switched on to the primary coil, and the consequent deflexion of the galvanometer was measured. The constant of the galvanometer was thus determined from the field in the primary coil and the dimensions of the secondary circuit in the usual manner. The resistance of the whole secondary circuit was $10 \cdot 80 \Omega$.

The deflexion of the galvanometer was read by means of a scale and telescope with a scale distance of $1.527 \mathrm{~m}$. The sensibility of the arrangement was such that one scaledivision corresponded to a change of 1.42 c.G.s. units of intensity of magnetization. To obtain a smooth motion of the galvanometer mirror due to the induction, it was found necessary that the two primaries as well as the two secondaries should have nearly the same dimensions respectively; the kick, which was observed when the dimensions of these coils were different, was probably due to the self-inductions and the capacities in these coils.

Compensation for the earth field was effected by a special coil of fine copper wire wound on a glass tube in a single layer. This coil fitted closely to the inside of the magnetizing coil and to the ontside of the secondary. It was fed by a current from two Daniel cells with adjustable resistance in the circuit.

The current in the primary circuit was measured by a Siemens and Halske ammeter with two shunts, $\frac{1}{9}$ and $\frac{1}{99^{*}}$ 'This was occasionally compared with a Kelvin's amperebalance.

The specimen to be tested was cut to a suitable length (about $27 \mathrm{~cm}$.) so that, if placed centrally, it might lie in a nearly uniform field of the magnetizing coil ; it was brazed 
at both its ends to thick rods of brass. The whole was hung. vertically in the axial line of the magnetizing coil, and consequently of the secondary coil, the upper rod being firmly clamped to the rigid frame above the coils. To the end of the lower rod a hook was attached; from this hung a flexible cord which, after passing through a system of two pulleys, was stretched by a weight, without imparting any injurious pendulum motion to the specimen. Near the end of the lower rod, a rigid pin was screwed on perpendicular to the rod. The ends of the pin fitted to the two $\mathrm{V}$-shaped grooves cut lengthwise and diametrically opposite to each other on the inside of a brass cylinder, which could be turned about a fixed vertical axis to any desired angle. The angle of twist was read by means of a graduated circle and the index attached to the torsion cylinder to $\frac{1}{20}$ of a degree. In the experiment of the tension effect, the above arrangement served to prevent any accidental twisting of the specimen without causing a sensible friction to the stretching.

\section{§2. Method of Experiments.}

Our procedure was usually made in the following order :The direct effect of the magnetizing coil on the galvanometer was tested first of all. The specimen was removed, the secondary circuit opened, and the maximum current was passed through the primary. If there were any constant deflexion of the galvanometer-mirror, the observer signalled to the experimenter, who adjusted the orientation of the compensating primary coil till the deflexion on breaking, making, or reversing the current was brought to zero.

Next the secondary circuit was closed, a strong current. was passed through the primary, while the observer was watching the galvanometer; the compensating secondary was slid within the primary till the ballistic deflexion was reduced to zero.

Next the compensation for the earth field was effected. For this purpose, the specimen was introduced into the magnetizing coil, clamped firmed and stretched by a suitable tension, care being taken to place the wire co-axially with the coils. The specimen was carefully demagnetized by reversals; the secondary was closed, a weak field excited in the primary, and the consequent deflexions noted. After a complete demagnetization, the same magnetizing current was passed in the opposite direction. If the two corresponding deflexions of the galvanometer were not equal to each other, the resistance in the compensating circuit was so adjusted, that: 
the reversal of the magnetizing field, if it was repeated two or three times, caused an equal deflexion of the galvanometer. This method was found to be very sensitive, a very small change of the current in the compensating system producing a decided inequality of the galvanometer deflexions in opposite directions.

The tension effect was first tried. To wipe out any uncertain remanent stress of the specimen, cycles of tensions, from zero to the greatest to be used for the specimen, were passed through before commencing any experiment. As a preliminary test of the working of the arrangement, a series of increasing fields was applied step by step under a constant tension, and the increase of magnetization was observed by the galvanometer. After a complete demagnetization, a weak field was applied and kept constant. While the observer was watching the galvanometer, the experimenter applied a series of increasing tensions step by step; the throw of the galvanometer at each step was recorded. Then the tension was decreased step by step, and the corresponding deflexions were sometimes noted. After passing through several eycles of the tensions, the observation was repeated. After a complete demagnetization, the procedure was repeated for another higher field and so on. The number of fields chosen was naturally large for the region where the change of magnetization was considerable, but few where it was small. The magnetizing current was found to remain nearly constant during an experiment, except in strong fields, where it was sometimes found to vary 2 or 3 per cent. The reading of the ammeter was always observed both before and after the experiment, and the mean was taken.

Instead of increasing the tension step by step, the maximum tension was often applied at once; but it was found that the consequent deflexion of the galvanometer was nearly the same as the sum of the deflexions obtained by the application of tension in successive steps.

Another series of experiments is possible in this direction. The specimen was demagnetized with the smallest initial tension; it was then magnetized, and then the deflexion of the galvanometer due to an additional weight was observed. After several alternate additions and removals of the additional weight, the changes of magnetization due to the addition and removal were observed. Then the demagnetization with the initial and added weights was effected, and the change of magnetization due to a second additional weight was measured, and so on.

Next, the magnetization under constant tension was determined. The specimen was first thoroughly demagnetized by 
reversals, loaded with the empty pan only. A series of successively increasing fields was applied step by step, and the throw of the galvanometer corresponding to each increment of the field was recorded. Demagnetization was again effected, after the specimen had been loaded with an additional tension, and the magnetization tested in the same way; and so on. In this way, the magnetization under different constant tensions was obtained.

The procedure in the experiments on the effect of torsion was similar. The torsion was increased step by step under a constant field, and the change of magnetization corresponding to each step was observed. The effect of cyclic twist was also investigated. The effect of the maximum twist applied at once does not differ from the sum of the deflexions obtained by graduated application of $t$ wists, as in the case of tension. The magnetization under constant torsions was next measured. These sets of experiments were repeated for several tensions nearly equal to those used in our previous experiments on the change of rigidity by magnetization.

The standardization of the ballistic galvanometer was made for each set of observations, though the constant remained fairly uniform during the whole investigation. Instead of using each time the special coil made for the standardization, we often used the compensating secondary coil for a set of experiments, recording the deflexions of the galvanometer corresponding to a series of magnetizing currents, and at the end of a set, the induction of this coil was compared with that of the standardizing coil. In this way, time and labour were economized, without the risk of introducing any sensible error in the estimation of the constant of the galvanometer.

\section{§3. Results of Experinents.}

The intensity of magnetization was calculated in the usual manner from the throw of the ballistic galvanometer with a known constant, the numbers of turns of the secondary and the standardizing coil, and the sections of the speciunen wire and the standardizing coil. The necessary correction for the reduction to tangent was made for considerable deflexions. The magnetizing field was calculated from the reading of the ammeter of known constant combined with the known number of turns of the coil. The demagnetizing force, though it was very small, was also taken into account. Tensions were all reduced to weights per square millimetre, and torsions to twists per unit length.

In the following pages, I denotes the intensity of magnetization, $\mathrm{H}^{\prime}$ the external field applied and $\mathrm{H}$ the internal or 
effective field, all expressed in c.G.s. units; $\mathrm{T}$ denotes the tension in grams per square millimetre, and $\tau$ the twist in minutes of arc per unit of length.

\section{Swedish Iron and Tungsten-Steel.}

The effect * of tension or of torsion on the magnetization of iron and steel is so well known that it is superfluous to enter into a detailed description of the effect. Only the general features of the change of magnetization will be given here. It will, however, be noticed that our investigation has one characteristic, that several effects of the stress on magnetization were studied on the same specimen with special attention to the order of applying the stress and the field. The specimens were also those on which strains caused by magnetization had been fully studied; hence the numerical results of the present experiment should be of some use to theoreticians, who have either already obtained, or may attempt to obtain, some reciprocal relations between magnetization and stress, so that they will be given in their proper places.

\section{(a) Change of Magnetization by Tension under Constant Field: $(\delta \mathrm{I}, \mathrm{T})_{\mathrm{H}}$.}

As will be seen from figs. $1 \& 3$ (Pl. I.), the change of magnetization $\delta \mathrm{I}_{i}$ due to the initial effect of loading increases up to a moderate field, and then decreases with it. In Swedish iron, curves $\left(\delta \mathrm{I}_{i}, \mathrm{~T}\right)_{\mathrm{I}}$ in weak fields initially bend upward, and after passing through an inflexion-point, the curvature changes sign. As the field is increased, the point of inflexion approaches the origin; in strong fields, $\delta I_{i}$ is very small, and the curve is nearly straight. In tungsten-steel, curve $\left(\delta \mathrm{I}_{i}, \mathrm{~T}\right)_{\text {r }}$ has a slight curvature for all fields.

In weak fields, the effect of removing the suspended weight is very small and slightly increases the magnetization. Subsequent loading causes an increase of magnetization; and unloading, a decrease. In strong fields, the initial and the cyclic effect of loading nearly coincide with each other. Curves $\left(\delta \mathrm{I}_{c}, \mathrm{~T}\right)_{\text {II }}$ for cyclic effects are given in figs. 2 and 4 in magnified scale. Curves $\left(\delta \mathrm{I}_{i}, \mathrm{H}\right)_{\mathrm{T}}$ as deduced from the initial effect of $\left(\delta \mathrm{I}_{i}, \mathrm{~T}\right)_{\mathrm{I}}$ are given in full lines in figs. 5,6 and 7 ; they rise and then fall steeply in low field, and afterward decrease slowly, cutting the axis of $\mathrm{H}$ at the Villari

* See Wiedemann's Electricität, iii. chap. 4; Ewing's 'Magnetic Jnduction,' chap. 9; Winkelmann, Handbuch der Physik, Zweite Auflage, $\mathrm{V}_{\cdot 1}$, pp. 301-307, 313-319. 
points. The decrease of magnetization reaches a maximum, and then gradually diminishes tending to approach zero, as the field is increased. This maximum decrease had been anticipated from the theory of magnetostriction * by Professor Nagaoka and one of us. Curves $\left(\delta \mathrm{I}_{i}, \mathrm{H}\right)_{\mathrm{T}}$ as deduced from $\left(\delta \mathrm{I}_{c}, \mathrm{~T}\right)_{\text {r }}$ of the cyclic effect rise only slightly in weak fields; but in strong fields they nearly coincide with curves $\left(\delta I_{i}, \mathrm{H}\right)_{\mathrm{r}}$ for the initial effect. The following are the numbers obtained by experiments.

\section{Swedish Iron.}

Initial $\mathrm{T}=152 \mathrm{gr} . / \mathrm{mm}^{2} ; t=16^{\circ} .5 \mathrm{C}$.

\begin{tabular}{|c|c|c|c|c|c|c|c|c|c|c|}
\hline \multirow{2}{*}{ H'. } & \multicolumn{2}{|c|}{$\mathrm{T}=1562 \mathrm{gr}$} & \multicolumn{2}{|c|}{$\mathrm{T}=3086 \mathrm{gr}}$. & \multicolumn{2}{|c|}{$\mathrm{T}=4648 \mathrm{gr}}$. & \multicolumn{2}{|c|}{$\mathrm{T}=6211 \mathrm{gr}$} & \multicolumn{2}{|c|}{$\mathrm{T}=7777 \mathrm{gr}$} \\
\hline & $\delta \mathrm{L}_{i}$ & $\delta \mathrm{I}_{c}$ & $\delta \mathrm{I}_{i}$ & $\delta \mathrm{I}_{c}$ & $\delta \mathrm{I}_{i}$ & $\delta \mathrm{I}_{G^{*}}$ & $\delta \mathrm{I}_{i}$ & $\delta \mathrm{I}_{c}$ & $\delta \mathrm{I}_{i}$ & $\delta \mathrm{I}_{c}$ \\
\hline $1 \cdot 25$ & 68 & 0.9 & $23 \cdot 2$ & 1.9 & 568 & $3 \cdot 2$ & $100 \cdot 9$ & $4 \cdot 5$ & 1463 & $6 \cdot 9$ \\
\hline 256 & 31.3 & 0.8 & $94 \cdot 4$ & 1.5 & 1844 & $2 \cdot 3$ & $275 \cdot 8$ & $3 \cdot 1$ & $343 \cdot 7$ & $6 \cdot 1$ \\
\hline $4 \cdot 37$ & $106^{\circ} 4$ & $0^{\circ} 1$ & 2262 & $-0 \cdot 2$ & $3 \div 69$ & $-0 \cdot 6$ & 397.8 & -10 & 444.0 & -08 \\
\hline 588 & 115.5 & $-1 \cdot 1$ & 197.8 & -1.9 & $251 \cdot 7$ & -28 & 2859 & -40 & 306.9 & -50 \\
\hline $7 \cdot 75$ & 687 & $-1 \cdot 7$ & $111 \cdot 1$ & -3.4 & 1409 & -50 & 1607 & -67 & $171 \cdot 6$ & $8 \cdot$ \\
\hline 11.90 & 253 & $-2 \cdot 6$ & $40 \cdot 7$ & $-5 \cdot 2$ & 51.0 & $-7 \cdot 9$ & $57 \cdot 3$ & $-10 \cdot 5$ & $61 \cdot 1$ & -13 \\
\hline $24 \cdot 18$ & 1.5 & $-3 \cdot 4$ & 1.8 & -67 & $1 \cdot 1$ & $-10 \cdot 2$ & $-0 \cdot 1$ & $-18 \cdot 9$ & -1.9 & -17 \\
\hline 3655 & $-2 \cdot 7$ & $-3 \cdot 8$ & $-5 \cdot 1$ & -7.8 & -75 & $-11 \cdot 6$ & -10.3 & -15.5 & -133 & -196 \\
\hline $97 \cdot 1$ & $-4 \cdot 0$ & $\ldots$ & $-7 \cdot 8$ & $\ldots$ & $-11 \cdot 6$ & $\ldots$ & $-14 \cdot 7$ & $\ldots$ & $-18 \cdot 2$ & \\
\hline $204 \cdot 7$ & -38 & $\ldots$ & $-7 \cdot 3$ & $\ldots$ & $-10 \cdot 8$ & $\ldots$ & $-14 \cdot 4$ & $\ldots$ & -18.1 & \\
\hline $366 \cdot 6$ & -27 & ... & $-5 \cdot 1$ & $\cdots$ & -80 & ... & $-10 \cdot 6$ & $\cdots$ & $-13 \cdot 3$ & \\
\hline
\end{tabular}

Tungsten-Steel.

Initial $\mathrm{T}=159 \mathrm{gr} . / \mathrm{mm} .^{2} ; t=14^{0} \cdot 2 \mathrm{C}$.

\begin{tabular}{|c|c|c|c|c|c|c|}
\hline \multirow{2}{*}{$\mathrm{H}^{\prime} \cdot$} & \multicolumn{2}{|c|}{$\mathrm{T}=1625 \mathrm{gr} . / \mathrm{mm} .2$} & \multicolumn{2}{|c|}{$\mathrm{T}=4837 \mathrm{gr} . / \mathrm{mm} .^{2}$} & \multicolumn{2}{|c|}{$\mathrm{T}=8092 \mathrm{gr} . / \mathrm{mm} .^{2}$} \\
\hline & $\delta \mathbf{I}_{i}$ & $\delta \mathrm{I}_{c}$ & $\delta \mathrm{T}_{i}$ & $\delta I_{c .}$ & $\delta \mathrm{I}_{i}$ & $\partial \mathrm{I}_{c}$ \\
\hline $4 \cdot 08$ & 04 & $0 \cdot 2$ & $2 \cdot 6$ & 0.4 & 6.5 & 0.7 \\
\hline $10 \cdot 6$ & 32 & 0.6 & 130 & 1 . & 27.7 & $3 \% 2$ \\
\hline 15.70 & 9.1 & 1.0 & $42 \cdot 1$ & 4.2 & $98 \cdot 4$ & $8 \cdot 1$ \\
\hline $19 \cdot 34$ & 400 & $2 \cdot 6$ & 1423 & 67 & 2149 & $11 \cdot 9$ \\
\hline 23.85 & $35 \cdot 3$ & $2 \cdot 0$ & $98: 3$ & $6 \cdot 1$ & $149 \cdot 1$ & $10^{9} 9$ \\
\hline $32 \cdot 16$ & $16 \cdot 2$ & 1.6 & 436 & 4.5 & $67 \cdot 6$ & $7 \cdot 9$ \\
\hline $43 \cdot 36$ & 6.9 & 1.2 & 210 & $3 \cdot 4$ & 32.8 & 56 \\
\hline 98.4 & 0.4 & 0.4 & 1.9 & $0 \cdot 4$ & $3 \cdot 1$ & $0 \cdot 1$ \\
\hline 210.0 & $-6 \cdot 4$ & $0 \cdot 0$ & $-1 \cdot 6$ & $-1 \cdot 3$ & -26 & -28 \\
\hline 341.0 & -0.3 & -0.3 & -1.5 & $-\overline{1} \cdot 7$ & $-2 \cdot 8$ & -34 \\
\hline
\end{tabular}

* Nagaoka and Honda, Jour. Sc. Coll, xiii. p. 69 (1900); Phil. Mag. xlix. p. 340 (1900). 
The effect of applying the maximum tension at once is nearly the same as the effect of the graduated applications of tensions.

The change of magnetization by tension under different initial loadings, where the demagnetization was always effected with the initial load, was also tested. For an equal increment $\delta \mathrm{T}$ of $\mathrm{T}, \delta \mathrm{I}_{i}$ decreases rapidly, as the initial $\mathrm{T}$ increases, whereas in the graduated applications of tension, $\delta \mathrm{I}_{i}$ increases nearly proportionally to $\delta \mathrm{T}$. The difference between these two values of $\delta I_{i}$ is considerable.

(b) Magnetization under Constant Tensions: $(\mathrm{I}, \mathrm{H})_{\mathrm{T}}$.

The magnetization increases rather rapidly in low fields and gradually approaches saturation. The effect of tension is, in its general features, similar to that obtained from $(\delta \mathrm{I}, \mathrm{T})_{\mathrm{H}}$. With low tensions, the increase of magnetization is considerably less than the value of the initial effect obtained from the latter experiment, while with high tensions, the contrary is true. These facts will be seen in the following tables and curves $(\delta \mathrm{I}, \mathrm{H})_{\mathrm{T}}$ in dotted lines in figs. 5 and 7.

\begin{tabular}{|c|c|c|c|c|c|}
\hline \multicolumn{2}{|c|}{$\mathrm{T}=152 \mathrm{gr} . / \mathrm{mur}^{2}$} & \multicolumn{2}{|c|}{$\mathrm{T}=1714 \mathrm{gr} . / \mathrm{mm}^{2}$} & \multicolumn{2}{|c|}{$\mathrm{T}=7929 \mathrm{gr} . / \mathrm{mm}^{2}$} \\
\hline H. & I. & $\mathrm{H}$. & I. & H. & I. \\
\hline 0.87 & 18.5 & 0.88 & $2 \cdot 3$ & 084 & $21 \cdot 3$ \\
\hline $1 \cdot 63$ & 527 & $2 \cdot 17$ & 91.7 & 1.63 & 598 \\
\hline $2 \cdot 89$ & $161 \% 3$ & 312 & 2355 & $3 \cdot 16$ & 2698 \\
\hline $4 \cdot 42$ & 549 & 367 & $4+1 \cdot 3$ & 386 & 4954 \\
\hline 6.35 & 835 & $46^{\prime}$ & 710 & 448 & 727 \\
\hline 848 & 1000 & $6 \cdot 4$ & $9+6$ & 657 & 982 \\
\hline 13.60 & 1141 & $15 \div 3$ & 1180 & 1351 & 1149 \\
\hline 2372 & 1229 & $24 \cdot 10$ & 1238 & 2385 & 1213 \\
\hline 3560 & 1275 & 3583 & 1230 & 35.88 & 1251 \\
\hline 58.39 & 1328 & 58.39 & 13331 & $54 \cdot 34$ & 1300 \\
\hline 127.3 & 1416 & 1266 & 1419 & 1248 & 1337 \\
\hline $219 \cdot 4$ & 1493 & 197.4 & 1478 & 1956 & 1448 \\
\hline 3192 & $15+2$ & $319 \cdot 1$ & 1547 & 296.8 & 1511 \\
\hline 386.5 & 1569 & 38300 & 1573 & $381 \cdot 9$ & 1547 \\
\hline
\end{tabular}


Tungsten-Steel.

$$
t=13^{\circ} \cdot 7 \mathrm{C} \text {. }
$$

\begin{tabular}{|c|c|c|c|c|c|c|c|}
\hline \multicolumn{2}{|c|}{$\mathbf{T}=159 \mathrm{gr} \cdot / \mathrm{mm}^{2}$} & \multicolumn{2}{|c|}{$\mathrm{T}=1784 \mathrm{gr} \cdot / \mathrm{mm} .^{2}$} & \multicolumn{2}{|c|}{$\mathrm{T}=4996 \mathrm{gr} . / \mathrm{mm} .^{2}$} & \multicolumn{2}{|c|}{$\mathrm{T}=8251 \mathrm{gr} / \mathrm{mm} .^{2}$} \\
\hline H. & I. & $\mathrm{HI}$. & I. & H. & I. & H. & I. \\
\hline $2 \cdot 33$ & $14 \cdot 4$ & $2 \cdot 35$ & $14: 9$ & 277 & $17 \cdot 6$ & $2 \cdot 33$ & $14 \cdot 2$ \\
\hline $4 \cdot 68$ & $31 \cdot 2$ & $5 \cdot 14$ & $35 \cdot 3$ & 554 & 38.5 & 460 & $29 \cdot 8$ \\
\hline 790 & 58.8 & $8 \cdot 8 \tilde{0}$ & $69 \cdot 1$ & 9.07 & 71.2 & 7.90 & 603 \\
\hline 1207 & 107.5 & $12 \cdot 41$ & $114 \cdot 2$ & 1287 & $123 \cdot 7$ & $12 \cdot 17$ & $1 \mathbf{1} 36$ \\
\hline 16.98 & $202 \cdot 3$ & 1676 & $212 \cdot 6$ & 1676 & $223 \cdot 6$ & 1680 & $241 \cdot 8$ \\
\hline 19.29 & 304.7 & 1900 & $3+1.3$ & 18.98 & 381.7 & 18.99 & $449 \cdot 0$ \\
\hline $22 \cdot 29$ & 532 & 2206 & 597 & 21.96 & 631 & 2.02 & 695 \\
\hline 27.73 & 788 & 2766 & 837 & 27.46 & 8.22 & 2764 & 877 \\
\hline 33.94 & 918 & 3360 & 95 & 3391 & 965 & 33.98 & 976 \\
\hline 41.70 & 1005 & 4233 & $10+1$ & 4272 & 1045 & $426 \mathrm{~s}$ & 1046 \\
\hline $63 \%$ & 1115 & 636 & 1144 & 632 & I 137 & $63 \% 2$ & 1133 \\
\hline $105 \cdot 2$ & 1207 & 1052 & 1233 & $105 \cdot 2$ & 1223 & $105 \cdot 6$ & 1215 \\
\hline 1583 & 1265 & 1584 & 1291 & 157.7 & 1270 & $158 \cdot 5$ & 1268 \\
\hline 2126 & 1308 & 2127 & 1327 & 2109 & 1311 & 2116 & 1304 \\
\hline 263.7 & 1328 & 2691 & 1342 & $267 \cdot 1$ & 1339 & 2687 & 1330 \\
\hline $345 \cdot 2$ & $13 \% 4$ & $346 \cdot 3$ & 1380 & 3438 & 1364 & $3+3.8$ & 1356 \\
\hline
\end{tabular}

\section{(c) Change of Magnetization by Twist under Different Tensions : $(\delta \mathrm{I}, \tau)_{\mathrm{H}, \mathrm{T}}$.}

In Swedish iron and tungsten-steel, the curves $\left(\delta \mathrm{I}_{i}, \tau\right)_{\mathrm{n}, \mathbf{T}}$ (figs. 8,10 , and 12) are similar to those for $(\delta \mathrm{I}, \mathrm{T})_{\mathrm{II}}$ for the initial effect. In weak fields where the twisting considerably increases the magnetization, the effect of the first untwisting is very small and slightly increases the magnetization. In Swedish iron, the cyclic effect of twisting (fig. 9) under low tensions is always to diminish magnetization. With high tensions (fig. 11), the effect has a singralar character : for a small twist, the magnetization increases, but for a large twist, it is diminished. In tungsten-steel, the cyclic effect of twist is similar to the cyclic effect of tension in Swedish iron, but in amount it is very small. Curves $\left(\delta \mathrm{I}_{i}, \mathrm{H}\right)_{\tau, \mathrm{T}}$ as deduced from $\left(\delta I_{i}, \tau\right)_{\mathrm{II}, \mathrm{T}}$ are given in figs. $13,14, \& 15$ (Pl. II.) in full lines. They closely resemble those for the tension effect, having points corresponding to the Villari points. With considerable twist, $\left(\delta \mathrm{I}_{c}, \mathrm{H}\right)_{\tau, \mathbf{r}}$ (figs. 13 and 14 ) is always negative. The experimental numbers are given in the table below :- 
Swedish Iron.

$\mathrm{T}=152 \mathrm{gr} . / \mathrm{mm} .^{2} ; t=15^{\circ} \cdot 6 \mathrm{C}$.

\begin{tabular}{|c|c|c|c|c|c|c|c|c|}
\hline \multicolumn{3}{|c|}{$\tau=31^{\prime} \cdot 9$} & \multicolumn{3}{|c|}{$\tau=42^{\prime} \cdot 9$} & \multicolumn{3}{|c|}{$\tau=69^{\prime} \cdot 8$} \\
\hline $\mathrm{H}^{\prime}$ & $\delta \mathrm{I}_{i}$ & $\delta \mathrm{I}_{c}$ & $\bar{H}^{\prime}$. & $\hat{\partial} \mathrm{I}_{i}$ & $\delta \mathbf{I}_{c^{\circ}}$ & $\mathrm{H}^{\prime}$. & $\delta \mathbf{I}_{i}$ & $\delta \mathrm{I}_{c}$ \\
\hline $1 \cdot 34$ & 70 & $-1 \cdot 0$ & $1 \cdot 26$ & $18 \cdot 6$ & $-1 \cdot 7$ & $1 \cdot 2 \cdot$ & $36 \cdot 8$ & $-4 \cdot 2$ \\
\hline $2 \cdot 43$ & 173 & -2.5 & $2 \cdot 47$ & $39 \cdot 4$ & -56 & $2 \cdot 38$ & $73 \cdot 2$ & $-11 \cdot 2$ \\
\hline $4 \cdot 12$ & $44 \cdot 3$ & $-5 \cdot 4$ & $4 \cdot 22$ & 908 & $-11 \cdot 2$ & $4 \cdot 24$ & 114.7 & -200 \\
\hline $5 \cdot 86$ & $44 \cdot 6$ & $-5 \epsilon$ & 573 & $84 \cdot 7$ & $-11 \cdot 2$ & $5 \cdot 73$ & 125.0 & $-21 \cdot 4$ \\
\hline $7 \cdot 79$ & $2 \pi 0$ & $-5 \cdot 3$ & 7.79 & $43 \cdot 5$ & $-11 \cdot 3$ & 7.87 & 46.7 & $-22 \cdot 5$ \\
\hline $13 \cdot 56$ & $4 \cdot 6$ & $-4 \cdot 4$ & $13 \cdot 60$ & 28 & $-10 \cdot 3$ & ] 3.59 & $-8 \cdot 3$ & -22.5 \\
\hline $24 \cdot 04$ & -0.7 & $-3 \cdot 1$ & $23 \cdot 78$ & $-4 \cdot 2$ & $-8 \cdot 2$ & $23 \cdot 74$ & $-14 \cdot 1$ & $-18 \cdot 3$ \\
\hline $48 \cdot 95$ & $-1 \cdot 3$ & $-1 \cdot 7$ & $42 \cdot 01$ & $-3 \cdot 9$ & $-4 \cdot 4$ & $39 \cdot 28$ & $-10-3$ & -11.5 \\
\hline $114 \cdot 4$ & -0.3 & -0.3 & $117 \div 0$ & $-i \cdot I$ & $-I \cdot 4$ & 116.8 & -2.5 & -30 \\
\hline $212 \cdot 7$ & -0.0 & -03 & $219 \cdot 0$ & 0.0 & -0.8 & 215.2 & $-1 \cdot 4$ & -1.4 \\
\hline $367 \cdot 1$ & $-0 \cdot 1$ & $-0 \cdot 1$ & $\ldots$ & $\ldots$ & ... & $370 \cdot 6$ & -0.4 & -0.9 \\
\hline
\end{tabular}

$\mathrm{T}=3238 \mathrm{gr} . / \mathrm{mm} \cdot{ }^{2} ; t=15^{\circ} \cdot 6 \mathrm{C}$.

\begin{tabular}{|c|c|c|c|c|c|c|c|c|c|c|c|}
\hline \multicolumn{3}{|c|}{$\tau=14^{t} \cdot 6$} & \multicolumn{3}{|c|}{$\tau=30^{\prime} \cdot 2$} & \multicolumn{3}{|c|}{$\tau=-58^{\circ}-5$} & \multicolumn{3}{|c|}{$T=8 i^{\prime} \cdot 8$} \\
\hline $\mathrm{H}^{\prime}$. & $\delta I_{i}$ & $\delta \mathrm{I}_{\epsilon}$ & $H^{\prime}$. & $\delta \mathrm{I}_{i}$. & $\delta \mathrm{I}_{e}$ & $\mathrm{H}^{\prime}$. & $\partial \mathrm{I}_{i}$. & $\delta \mathrm{I}_{c}$ & $\mathrm{H}^{\prime}$. & $\delta \mathrm{I}_{i}$ & $\delta I_{e}$ \\
\hline $1 \cdot 21$ & $5 \cdot 7$ & 0.6 & $1 \cdot 22$ & $30 \cdot 2$ & 33 & $1 \cdot 22$ & 125.2 & 3.8 & 1.21 & $182 \cdot 1$ & $-17 \cdot 1$ \\
\hline $2 \cdot 42$ & $22 \cdot 4$ & $2 \cdot 1$ & $2 \cdot 61$ & 116.8 & 6.4 & 254 & 296.0 & -2.0 & $2 \cdot 55$ & 885.0 & $-32 \cdot 2$ \\
\hline 4.02 & 73.7 & $3 \cdot 7$ & $4 \cdot 05$ & 194.0 & 57 & $4 \cdot 17$ & $328 \cdot 2$ & -70 & 4.03 & $401 \cdot 0$ & $-37 \cdot 2$ \\
\hline $5 \cdot 76$ & $47 \cdot 0$ & $3 \cdot 1$ & 5.78 & 1162 & 40 & $5 \cdot 76$ & 176.4 & -6.8 & $6 \cdot 00$ & 164.0 & -356 \\
\hline 7.76 & 273 & $2 \cdot 4$ & $7 \cdot 76$ & 56.4 & $3 \cdot 0$ & $7 \cdot 80$ & 86.5 & -63 & $7 \cdot 71$ & $76 \cdot 8$ & $-3: 6$ \\
\hline 1363 & 11.4 & $1 \cdot 4$ & 13.57 & 24.2 & 1.7 & $13 \cdot 63$ & $29 \cdot 4$ & $-4 \cdot 7$ & $13 \cdot 63$ & $16 \cdot 6$ & $-22 \cdot 8$ \\
\hline $22 \cdot 92$ & 5.8 & $1 \cdot 3$ & $21 \cdot 70$ & 124 & 1.1 & $22 \cdot 47$ & 138 & -30 & $21 \cdot 42$ & 57 & $-14 \cdot 4$ \\
\hline $49 \cdot 11$ & $1 \cdot 4$ & 0.9 & $49 \cdot 15$ & $3 \cdot 1$ & $0 \cdot 6$ & $49 \cdot 21$ & 28 & $-1 \cdot 1$ & 48.90 & 1.4 & -55 \\
\hline 113.5 & 0.4 & 00 & 1058 & 1.0 & 0.0 & 1112 & 10 & -01 & 1050 & -1.0 & -18 \\
\hline $210^{\circ}$ & 0.0 & $0 \cdot 0$ & 2127 & $0 \cdot 1$ & 0.0 & $214 \cdot 1$ & 0.0 & $0 \cdot 0$ & 2102 & -0.3 & -0.4 \\
\hline
\end{tabular}


Effect of Stress on Magnetization.

Tungsten-Steel.

$\mathrm{T}=3371 \mathrm{gr} . / \mathrm{mm}^{2} ; t=13^{\circ} \cdot 7 \mathrm{C}$.

\begin{tabular}{|c|c|c|c|c|c|c|c|c|c|}
\hline \multicolumn{2}{|c|}{$\mathbf{H}^{\prime}=4 \cdot 01$} & \multicolumn{2}{|c|}{$\mathbf{H}^{\prime}=10 \cdot 72$} & \multicolumn{2}{|c|}{$\mathrm{H}^{\prime}=14 \cdot 53}$. & \multicolumn{2}{|c|}{$\mathrm{H}^{\prime}=18 \cdot 39}$. & \multicolumn{2}{|c|}{$\mathrm{H}^{\prime}=22 \cdot 40$} \\
\hline$\tau$. & $\delta \mathrm{I}_{i}$ & T. & $\delta I_{i}$ & $\tau$ & $\delta \mathrm{I}_{i}$ & $\tau$ & $\delta \mathrm{I}_{i}$ & $\tau$ & $\delta \mathrm{I}_{i}$. \\
\hline $18 \cdot 4^{\prime}$ & $0 \cdot 9$ & $12 \cdot 0^{\prime}$ & $2 \cdot 2$ & $129^{\prime}$ & $4 \cdot 0$ & $13 \cdot 8^{\prime}$ & 8.9 & $17 \cdot 6^{\prime}$ & 13.4 \\
\hline $39 \cdot 8$ & $2 \cdot 2$ & 47.5 & $7 \cdot 1$ & $39 \cdot 1$ & $14 \cdot 4$ & $38 \cdot 4$ & $32 \cdot 6$ & $41 \cdot 4$ & $37 \cdot 0$ \\
\hline 69.2 & $4 \div 9$ & $69 \cdot 2$ & $15 \cdot 7$ & $69 \cdot 2$ & $29 \cdot 3$ & 69.5 & $69 \cdot 9$ & $69 \cdot 2$ & 633 \\
\hline & 0.1 & & 0 & & 0 & & -03 & & $-0 \cdot 3$ \\
\hline $\mathrm{H}^{\prime}=$ & $30 \cdot 07$ & $\Pi^{\prime}=$ & 605 & $\mathrm{H}^{\prime}=$ & $98 \cdot 8$. & $\mathrm{HI}^{\prime}=$ & $209 \cdot 0$. & $\mathrm{H}^{\prime}=$ & $36 \pm 0$ \\
\hline$\tau$ & $\delta \mathrm{I}_{i}$ & $\tau$. & $\delta \mathrm{I}_{i}$ & $\tau$. & $\delta \mathrm{I}_{i}$. & $T$. & $\delta \mathrm{I}_{i}$ & $\tau$. & $\delta \mathrm{I}_{i}$. \\
\hline $12 \cdot 9^{\prime}$ & $3 \cdot 4$ & $13 \cdot 4^{\prime}$ & $1 \cdot 3$ & $14 \cdot 5^{\prime}$ & 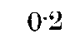 & $13 \cdot 0^{\prime}$ & 0.0 & $21 \cdot 4^{\prime}$ & 0.0 \\
\hline $34 \cdot 8$ & $12 \cdot 4$ & $48 \cdot 4$ & $6 \cdot 6$ & $42 \cdot 7$ & 15 & $47 \cdot 6$ & 00 & - & - \\
\hline $69 \cdot 2$ & $27 \cdot 2$ & $69 \cdot 2$ & $9 \cdot I$ & $69 \cdot 2$ & 23 & $69 \cdot 2$ & $-0 \cdot 1$ & $69 \cdot 2$ & 0.0 \\
\hline & -0.7 & & -08 & & $-1 \cdot 0$ & & -0.3 & & $-0 \cdot 2$ \\
\hline
\end{tabular}

The numbers in the last row give the cyclic effect of twist corresponding to the maximum twist.

The effect of the graduated application of twist does not sensibly differ from that of applying the maximum twist at once.

\section{(d) Magnetization under Constant Twist combined with Tensions : $(\mathrm{I}, \mathrm{H})_{\tau, \mathrm{H}}$.}

The effect of a constant twist on magnetization is very small ; in a small twist, the magnetization slightly increases, but above a moderate twist, it is decreased by twisting. With a moderate twist, curves $(\delta \mathrm{I}, \mathrm{H})_{\tau, \mathbf{r}}$ as deduced from $(\mathrm{I}, \mathrm{H})_{\tau, T}$ have an opposite sign to those deduced from $\left(\delta \mathrm{I}_{i}, \tau\right)_{\mathrm{H}, \mathrm{T}}$; but they have the same sign as curves $\left(\delta \mathrm{I}_{c}, \mathrm{H}\right)_{\tau, \mathrm{T}}$ obtained from $\left(\delta \mathrm{I}_{c}, \tau\right)_{\mathrm{H}, \mathrm{T}}$. The following tables and the dotted curves in figs. $13,14, \& 15$ (PI. II.) will show these changes of magnetization. 
Swedish Iron.

$\mathrm{T}=152 \mathrm{gr} . / \mathrm{mm}^{2} ; t=15^{\circ} \cdot 7 \mathrm{C}$.

\begin{tabular}{|c|c|c|c|c|c|}
\hline \multicolumn{2}{|c|}{$\tau=0}$. & \multicolumn{2}{|c|}{$\tau=31^{\prime} \cdot 9$} & \multicolumn{2}{|c|}{$\tau=70^{\prime} \cdot 3$} \\
\hline H. & 1. & $\mathrm{H}$. & I. & H. & J. \\
\hline $0 \cdot 77$ & $16 \cdot 8$ & $0 \cdot 68$ & $14 \cdot 8$ & 0.68 & $12 \cdot 5$ \\
\hline 1.68 & 47.8 & 1.29 & 333 & 1.39 & 325 \\
\hline $2 \cdot 38$ & $95 \cdot 1$ & $2 \cdot 29$ & 79.5 & $2 \cdot 29$ & 660 \\
\hline 349 & 2569 & 331 & 2110 & 2.93 & 1293 \\
\hline 4.05 & 406.1 & $4 \cdot 21$ & 4162 & $3 \cdot \varepsilon 6$ & $257 \cdot 7$ \\
\hline 4.69 & 571 & 5.06 & 632 & $4 \cdot 49$ & $410 \cdot 1$ \\
\hline 5.91 & 776 & 651 & 838 & $5 \cdot 74$ & 683 \\
\hline $7 \cdot 58$ & 933 & $8 \cdot 33$ & 975 & 868 & 945 \\
\hline $11 \cdot 45$ & $109 t$ & 12.20 & 1110 & $12 \cdot 47$ & 1064 \\
\hline 2051 & 1215 & 2064 & 1211 & $20 \cdot 65$ & 1173 \\
\hline $30 \cdot 81$ & 1266 & 31.08 & 1264 & $31 \cdot 11$ & 1238 \\
\hline 5000 & 1320 & $50 \cdot 83$ & 1319 & 50.91 & 1299 \\
\hline 110.8 & 1408 & 1102 & 1409 & $111 \cdot 1$ & 1403 \\
\hline 1735 & 1466 & 172.8 & 1467 & 1735 & 1462 \\
\hline 2635 & 1524 & 261.0 & 1526 & 2635 & 1521 \\
\hline $358 \cdot 1$ & 1568 & $357 \cdot 5$ & 1569 & $359 \cdot 3$ & 1566 \\
\hline
\end{tabular}

$\mathrm{T}=3238 \mathrm{gr} . / \mathrm{mn}^{2} ; t=15^{\circ} \cdot 6 \mathrm{C}$.

\begin{tabular}{|c|c|c|c|c|c|c|c|c|c|}
\hline \multicolumn{2}{|c|}{$\tau=0}$. & \multicolumn{2}{|c|}{$\tau=14^{\prime} \cdot 2$} & \multicolumn{2}{|c|}{$\tau=29^{\prime} \cdot 3$} & \multicolumn{2}{|c|}{$\tau=5 \mathrm{~s}^{\prime} \cdot 3$} & \multicolumn{2}{|c|}{$r=88^{r} \cdot 3$} \\
\hline H. & I. & $\mathrm{H}$. & I. & H. & I. & H. & I. & H. & I. \\
\hline 0.66 & 159 & 0.68 & 16.8 & 0.71 & $17 \cdot 2$ & $0 \cdot 19$ & $3 \cdot 1$ & $0.76^{\circ}$ & 16.5 \\
\hline 1.30 & & 1.29 & $41 \% 2$ & $1 \cdot 31$ & 41 . & 132 & $37 \cdot 2$ & 1 . & 43 \\
\hline $2 \cdot 23$ & 98 & 1.91 & 793 & 219 & 1004 & $2 \cdot 20$ & $94 \cdot 3$ & $2 \cdot 25$ & 79 \\
\hline $3 \cdot 15$ & $257 \cdot 7$ & $2 \cdot 73$ & $19 \cdot \pi$ & 2.85 & 1928 & 2.95 & $168 \cdot 4$ & $2 \cdot 98$ & 145 \\
\hline $3 \cdot 77$ & 4509 & 351 & $424 \cdot 0$ & 354 & 4184 & 3.78 & $351 \%$ & $3 \cdot 82$ & 300 \\
\hline $4 \cdot 48$ & 719 & $4 \cdot 19$ & 635 & 433 & 708 & $4 \cdot 60$ & 645 & $4 \cdot 81$ & 609 \\
\hline 5.69 & 940 & $5 \cdot 5 \pi$ & 929 & 5.75 & 963 & 6.05 & 925 & $6 \cdot 57$ & 890 \\
\hline $6 \cdot 75$ & 1028 & 667 & $10 \div 3$ & $7 \cdot 19$ & 1064 & 7.99 & 1048 & $8 \cdot 25$ & 1005 \\
\hline $13 \cdot 74$ & 1196 & 15.27 & 1210 & 1475 & 1219 & $15 \cdot 30$ & 1196 & $12:$ & 1114 \\
\hline 20.98 & 1250 & 21.09 & 1248 & $21 \cdot 36$ & 1265 & $21 \cdot 21$ & $12+2$ & 2074 & 1206 \\
\hline 31.06 & 1292 & 30.99 & $12 \varangle 8$ & 31.57 & 1305 & 30.79 & 128 & 31.00 & 1265 \\
\hline $50 \cdot 50$ & 1342 & $51 \cdot 23$ & 1341 & $51 \cdot 65$ & 1356 & $50 \cdot 91$ & 1346 & 5062 & 1330 \\
\hline 1157 & 1436 & 1157 & 1436 & $113 \cdot 3$ & 1449 & 11244 & 1444 & $111 \cdot 9$ & 1433 \\
\hline $180 \div$ & 1499 & $180 \cdot 5$ & 1499 & 177.4 & 1511 & 175.9 & 1506 & 175.1 & 1496 \\
\hline $275 \cdot 2$ & 1562 & 274.7 & 1561 & 2708 & 1573 & $267 \cdot 3$ & 1568 & $266 \cdot 3$ & 1560 \\
\hline 376.2 & 1610 & $376 \cdot 1$ & 1610 & 370.4 & 1621 & 3656 & 1616 & $365^{\circ} 6$ & 1608 \\
\hline
\end{tabular}


Tungsten-Steel.

$\mathrm{T}=3371 \mathrm{gr} . / \mathrm{mm}^{2} ; t=13^{\circ} \cdot 7 \mathrm{C}$.

\begin{tabular}{|c|c|c|c|}
\hline \multicolumn{2}{|c|}{$\tau=0}$. & \multicolumn{2}{|c|}{$\tau=69^{\prime} \cdot 2}$. \\
\hline H. & I. & H. & I. \\
\hline 2.71 & 167 & $2 \cdot 2 \cdot 2$ & $13 \cdot 4$ \\
\hline $4 \cdot 68$ & $31 \cdot 7$ & $4 \cdot 61$ & 29.8 \\
\hline$\overline{7} .98$ & 605 & 10.56 & 870 \\
\hline $12 \cdot 03$ & $109 \cdot 0$ & $14.7 \%$ & $153 \%$ \\
\hline $16 \cdot 80$ & 2226 & 1680 & $209 \cdot 8$ \\
\hline 19.03 & $36+2$ & 18.08 & $359 \cdot 2$ \\
\hline 22.04 & 630 & 21.97 & 622 \\
\hline 27.62 & 844 & 27.45 & 840 \\
\hline $34 \cdot 18$ & 958 & $33 \cdot 87$ & 958 \\
\hline $50 \div 6$ & 1083 & $42 \cdot+1$ & 1038 \\
\hline 859 & 1185 & $63 \cdot 2$ & 1136 \\
\hline $132 \cdot 2$ & 1247 & $105 \cdot 6$ & 1223 \\
\hline 179.8 & 1272 & 1584 & 1279 \\
\hline $210 \cdot 5$ & 1307 & $212 \cdot 4$ & 1318 \\
\hline $267 \cdot 7$ & 1334 & $269 \cdot 1$ & 1341 \\
\hline $344 \cdot 1$ & 1350 & 3438 & 1366 \\
\hline
\end{tabular}

From the results thus far described, it may be concluded that in Swedish iron and tungsten-steel, the final magnetization is affected in no inconsiderable degree by the order of magnetizing and straining. This fact stands parallel to the result of our previous experiment that in these metals, the change of elastic constants by magnetization is considerably affected by the order of applying the stress and the magnetizing field.

\section{Niokel.}

The effect of stress on the magnetization of nickel has been thoroughly studied by several physicists, so that there remains little to be studied about the effect. Our present investigation has, however, this characteristic, that several effects of stress on magnetization were studied with the same specimen over a wide range of the magnetizing field and with special attention to the hysteresis effect.

(a) Change of Magnetiation by Tension under Constant Field: $(\delta \mathrm{I}, \mathrm{T})_{\mathrm{rr}}$.

The initial effect of loading on magnetization in very weak fields is an increase of magnetization by low tension, and a decrease by high tension; but the cyclic effect is always a decrease, unlike the Villari reversal in iron. Above 2 c.G.s. 
units, however, the initial and the cyclic effects are always a decrease of magnetization.

In low tension, $\delta I_{i}$ or $\delta \mathrm{I}_{c}$ decreases almost proportionally with $\mathrm{T}$; as $\mathrm{T}$ is increased, the rate of decrease becomes great, and after passing through an inflexion-point it begins to decrease, as shown in fig. 16. As the field is increased, the decrease of magnetization passes through its maximum. Except in weak fields, the cyclic effect $\left(\delta \mathrm{I}_{c}, \mathrm{~T}\right)_{\text {н }}$ (fig. 16, dotted lines) fairly coincides with the initial effect. Curves $\left(\delta \overline{\mathrm{I}}_{i}, \mathrm{H}\right)_{\mathrm{T}}$ deduced from $\left(\delta \mathrm{I}_{i}, \mathrm{~T}\right)_{\mathrm{H}}$ are given in fig. 17 , in full lines. In weak fields, they fall steeply and then gradually rise; as the field is further increased, they slowly tend to approach the axis of the field.

Nickel.

Initial $\mathrm{T}=167 \mathrm{gr} . / \mathrm{mm} .{ }^{2} ; \quad t=14^{\mathrm{O}} \cdot 3 \mathrm{C}$.

\begin{tabular}{|c|c|c|c|c|c|c|c|c|c|c|}
\hline \multirow{2}{*}{$\mathbf{H}^{\prime}$} & \multicolumn{2}{|c|}{$\mathrm{T}=855 \mathrm{gr} . / \mathrm{mm}^{2}{ }^{2}$} & \multicolumn{2}{|c|}{$\mathrm{I}=17 \mathrm{II} \mathrm{gr}$} & \multicolumn{2}{|c|}{$\mathrm{T}=3421 \mathrm{gr}}$. & \multicolumn{2}{|c|}{$\mathrm{T}=5089 \mathrm{gr}$} & \multicolumn{2}{|c|}{$\mathrm{T}=85 \mathrm{l3} \mathrm{gr}$} \\
\hline & $\delta \mathrm{I}_{i}$ & $\delta \mathrm{I}_{c}$ & $\delta \mathrm{I}_{i}$ & $\hat{\delta} I_{c^{*}}$ & $\delta \mathbf{I}_{i}$ & $\delta \mathrm{I}_{c}$ & $\delta \mathrm{I}^{i}$ & $\delta \mathrm{I}_{c^{*}}$ & $\delta \mathrm{I}_{i}$ & $\delta \mathrm{I}_{c}$ \\
\hline \begin{tabular}{|c|}
$1 \cdot 14$ \\
2.35 \\
3.89 \\
$10 \cdot 7$ \\
$31 \cdot 66$ \\
62.54 \\
$135 \cdot 2$ \\
205.5 \\
364.7 \\
\end{tabular} & $\begin{array}{r}+66 \\
-58 \\
-10.9 \\
-234 \\
-233 \\
-12.4 \\
-4.8 \\
-20 \\
-03\end{array}$ & $\mid \begin{array}{c}-13.5 \\
-18.4 \\
-20.2 \\
-28.0 \\
-24 \cdot 5 \\
-12.7 \\
-4.6 \\
-2.0 \\
\ldots\end{array}$ & $\begin{array}{l}+9.7 \\
-18.4 \\
-29.2 \\
-52.5 \\
-54.0 \\
-29.5 \\
-10.6 \\
-4.6 \\
-0.6\end{array}$ & $\begin{array}{c}-32.2 \\
-42.8 \\
-47.7 \\
-58.7 \\
-55.1 \\
-29.5 \\
-10.7 \\
-4.9 \\
\ldots\end{array}$ & $\begin{array}{r}+\quad 35 \\
-\quad 62 \cdot 7 \\
-83.0 \\
-119 \cdot 2 \\
-1228 \\
-\quad 76 \cdot 0 \\
-\quad 25 \cdot 8 \\
-\quad 11 \cdot 3 \\
-\quad 2 \cdot 1\end{array}$ & $\begin{array}{c}-81 \cdot 0 \\
-102 \cdot 6 \\
-109 \cdot 4 \\
-129 \cdot 3 \\
-121 \cdot 2 \\
-76 \cdot 2 \\
-26 \cdot 1 \\
-12 \cdot 1 \\
\ldots\end{array}$ & $\begin{array}{r}-4 \cdot 1 \\
-85 \cdot 5 \\
-113 \cdot 8 \\
-170 \cdot 2 \\
-189 \cdot 2 \\
-129 \cdot 6 \\
-\quad 46 \cdot 1 \\
-\quad 20 \cdot 5 \\
-\quad 4 \cdot 1\end{array}$ & $\begin{array}{r}-99.4 \\
-128.1 \\
-143.4 \\
-181.8 \\
-185.5 \\
-129.9 \\
-47.5 \\
-21.3 \\
\ldots\end{array}$ & $\begin{array}{l}-9.2 \\
-97 \cdot 8 \\
-132.1 \\
-221 \cdot 3 \\
-267.5 \\
-235.5 \\
-113.2 \\
-49 \cdot 3 \\
-11.3\end{array}$ & $\begin{array}{c}-108.6 \\
-137.9 \\
-1636 \\
-223 \cdot 2 \\
-261 \cdot 1 \\
-234.6 \\
-114.4 \\
-49.9 \\
\ldots\end{array}$ \\
\hline
\end{tabular}

The effect of applying the maximum tension at once is nearly the same as that of the graduated applications of tension.

The change of magnetization by tension under different initial loadings, where the demagnetization was always effected with the initial load, was also tested. For the same increment $\delta \mathrm{T}$ of $\mathrm{T}, \delta \mathrm{I}$ is in a marked degree less than that in the last experiment.

(b) Magnetization under Constant Tensions: $(\mathrm{I}, \mathrm{H})_{\mathrm{T}}$.

The magnetization increases steeply in low fields, and after passing through an inflexion-point gradually approaches saturation. The effect of tension is considerably large and always to diminish magnetization; curves $(\mathrm{I}, \mathrm{H})_{\mathbf{r}}$ become less steep in weak fields with the increasing tension, and tend to approach each other in strong fields. If we compare 
$\delta$ I obtained from $(\mathrm{I}, \mathrm{H})_{\mathrm{T}}$ with that from $(\delta \mathrm{I}, \mathrm{T})_{\mathrm{H}}$ for the same values of $\mathrm{H}$ and $\mathrm{T}$, the former is found to be numerically a little greater than the latter. The dotted line in fig. 17 represents the values of $\delta \mathrm{I}$ as deduced from $(\mathrm{I}, \mathrm{H})_{\mathrm{r}}$.

Nickel.

$t=14^{\circ} \cdot 6 \mathrm{C}$.

\begin{tabular}{|c|c|c|c|c|c|c|c|c|c|c|c|}
\hline \multicolumn{2}{|c|}{$\mathrm{T}=16 \mathrm{gr} . / \mathrm{mm}}$. & \multicolumn{2}{|c|}{$\mathrm{T}=1022 \mathrm{gr}$} & \multicolumn{2}{|c|}{$\mathrm{T}=1878 \mathrm{gr}}$. & \multicolumn{2}{|c|}{$\mathbf{T}=3588 \mathrm{gr}}$. & \multicolumn{2}{|c|}{$\mathrm{T}=5256 \mathrm{gr}$} & \multicolumn{2}{|c|}{$\mathrm{T}=8680 \mathrm{gr}}$. \\
\hline II. & I. & H. & I. & H. & I. & $\mathrm{H}$. & $\mathrm{I}$. & $\mathrm{H}$. & I. & H. & I. \\
\hline 0.93 & 93 & 0.92 & $10 \cdot 0$ & 0.93 & $9 \cdot 1$ & 0.86 & $5 \cdot 0$ & $1 \cdot 66$ & $7 \cdot 6$ & 206 & $5 \cdot 8$ \\
\hline 16 & $82 \cdot 3$ & 202 & 739 & 1.91 & 38.9 & 1.76 & 118 & 350 & 167 & 441 & 129 \\
\hline $3 \cdot 19$ & $147 \cdot 6$ & $3: 36$ & 131.3 & $3 \cdot 24$ & $91 \cdot 1$ & 3.44 & $26 \cdot 8$ & $6 \cdot 64$ & 34.8 & $7 \cdot 78$ & $23 \cdot 4$ \\
\hline 4.12 & $170 \cdot 4$ & 4.02 & 154.4 & 4.43 & 1158 & $4 \cdot 81$ & 420 & 1076 & 60.4 & $13 \cdot 38$ & $41 \cdot 7$ \\
\hline 662 & 216.7 & $6 \cdot 65$ & $191 \cdot 2$ & 670 & $151 \cdot 6$ & $7 \cdot 75$ & $73 \cdot 4$ & $15 \cdot 96$ & 938 & $26 \cdot 01$ & $81 \cdot 5$ \\
\hline $10 \cdot 48$ & 2668 & $9 \cdot 66$ & 2265 & 1052 & $194 \cdot 2$ & $12 \cdot 14$ & $115 \cdot 1$ & & & & \\
\hline $16 \cdot 14$ & 316.6 & 1554 & $279 \cdot 3$ & $15 \cdot 72$ & $237 \cdot 1$ & 17.98 & $159 \cdot 3$ & $26 \cdot 56$ & $144: 3$ & & \\
\hline 2631 & 373.0 & $26 \cdot 30$ & 3430 & $26 \cdot 32$ & 301.2 & $27 \cdot 18$ & 214.5 & $39 \cdot 31$ & 2028 & $39 \cdot 24$ & $119 \cdot 3$ \\
\hline 38.88 & $413 \cdot 4$ & 39.09 & 3924 & $39 \cdot 11$ & 3546 & $39 \cdot 62$ & 270.9 & 629 & $291 \cdot 6$ & 59.8 & 176.4 \\
\hline 625 & 4660 & $62 \cdot 9$ & 444.5 & $63 \cdot 2$ & 4208 & $63 \cdot 4$ & $352 \cdot 0$ & $106 \cdot 3$ & $392 \cdot 1$ & 1056 & 2968 \\
\hline 1335 & 4900 & 1339 & $491 \cdot 4$ & $13+\overline{9}$ & $482 \cdot 8$ & I 38.4 & 451.0 & $186^{\circ} \cdot 1$ & 460.5 & 185.8 & $\$ 20.0$ \\
\hline 2057 & 5063 & 2061 & 508.2 & 2068 & 5027 & 2067 & 4797 & 211.7 & 4708 & $227 \cdot 1$ & 4495 \\
\hline 2698 & 5110 & 269.9 & $515 \%$ & $270 \cdot 5$ & 5116 & 2704 & $491 \cdot 5$ & $287^{\circ} 0$ & 487.9 & 284.1 & 4730 \\
\hline 3666 & 514.5 & 367.9 & 5200 & 3687 & 5176 & 367.3 & $499 \div 5$ & $358 \cdot 9$ & 1958 & 354.0 & 488.7 \\
\hline
\end{tabular}

(c) Change of Magnetization ly Twist under Different Tensions : $(\delta \mathrm{I}, \tau)_{\mathrm{H}, \mathrm{r}}$.

In weak fields the magnetization is increased by twist, but in strong fields it is slightly diminished. As shown in figs. $18 \& 19$ (Pl. II.), the curves $(\delta \mathrm{I}, \tau)_{\mathrm{H}, \mathrm{r}}$ bend slightly towards the axis of the twist; the curvatures become less as the tension is increased. Except in weik fields, the initial effect is inconsiderable; it also becomes less as the tension is increased.

Curves $\left(\delta I_{i}, \mathrm{H}\right)_{\tau}$, from $\left(\delta \mathrm{I}_{i}, \tau\right)_{\mathrm{Ir}, \mathrm{T}}$ are drawn in figs. 20 and 21 in full lines; they have steep positive maxima from which the curves slope down gradually to the higher field, cut the axis of $\mathrm{H}$, become negative, and after passing through very inconspicuous negative maxima, very slowly bend towards the axis; the maxima become flatter with the greater tensions, and the positions of the maxima as well as the points of intersection with the axis move toward higher fields with increasing tension. The course of the curves is thus quite similar to that of curves $(\delta I, H)_{T}$ in the case of iron. The following tables give some of the numerical data obtained.

Phil. Mo.g. S. 6. Vol. 14. No. 79. July $1907 . \quad$ G 
Nickel.

$\mathrm{T}=1197 \mathrm{gr} . / \mathrm{mm}^{2} ; \quad t=14^{0.9 \mathrm{C} .}$

\begin{tabular}{|c|c|c|c|c|c|c|c|c|c|c|c|}
\hline \multicolumn{4}{|c|}{$\mathrm{H}^{\prime}=0 \cdot 68$} & \multicolumn{4}{|c|}{$\mathrm{H}^{\prime}=1 \cdot 07$} & \multicolumn{4}{|c|}{$\mathrm{H}^{\prime}=2 \cdot 31$} \\
\hline$\tau$ & $\delta \mathbf{I}_{i}$ & $\tau$. & $\delta \mathrm{I}_{c}$ & $\tau$ & $\delta \mathrm{I}_{i}$ & $\tau$. & $\delta \mathrm{I}_{G}$ & r. & $\delta \mathrm{I}_{i}$ & $T$. & $\delta \mathrm{I}_{c}$ \\
\hline $8 \cdot 4^{\prime}$ & 221 & $9 \cdot 1^{3}$ & $34 \cdot 2$ & $160^{\prime}$ & $105 \cdot 3$ & $12 \cdot 2^{\prime}$ & 509 & $16 \div 5^{\prime}$ & 278 & $13 \cdot 1^{\prime}$ & $57 \cdot 2$ \\
\hline $21 \cdot 8$ & $72 \cdot 8$ & $23 \cdot 6$ & $91 \cdot 7$ & 26.9 & $158 \cdot 3$ & $28 \cdot 9$ & $109 \cdot 4$ & $34 \cdot 4$ & $95 \cdot 2$ & $30 \cdot 4$ & $121 \cdot 6$ \\
\hline $36 \cdot 9$ & $130 \cdot 6$ & $41 \cdot 8$ & $136 \cdot 3$ & 40.7 & $196 \cdot 6$ & $41 \cdot 8$ & 1375 & $46 \cdot 3$ & $152 \cdot 3$ & $43 \cdot 3$ & $150 \cdot 9$ \\
\hline $54 \cdot 2$ & $188 \cdot 8$ & $55 \cdot 2$ & 1556 & $54 \cdot 0$ & $221 \cdot 2$ & $58 \cdot 3$ & $161 \cdot 0$ & $63 \cdot 1$ & $216: 3$ & $58 \cdot 7$ & 1749 \\
\hline $69 \cdot 2$ & $221 \cdot 1$ & $69 \cdot 2$ & 171.9 & $69 \cdot 3$ & $239 \cdot 5$ & $69 \cdot 0$ & $172 \cdot 9$ & 69.4 & 2325 & $69 \cdot 3$ & $187 \cdot 1$ \\
\hline \multicolumn{12}{|c|}{ - } \\
\hline \multicolumn{4}{|c|}{$\mathrm{H}^{\prime}=3 \cdot 91$} & \multicolumn{4}{|c|}{$\mathbf{H}^{\prime}=10 \cdot 62$} & \multicolumn{4}{|c|}{$\mathrm{H}^{\prime}=23 \cdot 22$} \\
\hline$\tau$. & $\delta \mathrm{I}_{i}$ & $\tau$ & $\delta \mathrm{I}_{c}$ & $\tau$. & $\delta \mathrm{I}_{i}$ & $\pi$ & $\delta \mathrm{I}_{\boldsymbol{c}}$ & $T$. & $\delta \mathrm{I}_{i}$ & $\tau$. & $\delta \mathrm{I}_{c}$ \\
\hline $10 \cdot 2^{\prime}$ & $60 \cdot 2$ & $8.0^{\prime}$ & $29 \cdot 3$ & $10^{\circ} 8^{\prime}$ & 456 & $7 \cdot 1^{\prime}$ & 138 & $8 \cdot 8^{\prime}$ & 46 & $140^{\prime}$ & $4 \cdot 6$ \\
\hline $23 \cdot 7$ & $121 \cdot 0$ & $26 \cdot 3$ & $91 \cdot 8$ & $22 \cdot 6$ & $75 \cdot 4$ & $22 \cdot 7$ & $48 \cdot 0$ & 236 & $20 \cdot 0$ & $31 \cdot 7$ & 156 \\
\hline $36 \cdot 0$ & $151 \cdot 9$ & $38 \cdot 2$ & $117 \cdot 6$ & $37 \cdot 2$ & 1060 & 39.5 & $73 \cdot 2$ & $38 \cdot 3$ & $30 \cdot 3$ & $\ldots$ & $\ldots$ \\
\hline $53 \cdot 5$ & $179 \cdot 8$ & $48 \cdot 7$ & 1338 & $50 \cdot 7$ & $122 \cdot 5$ & 540 & $88 \cdot 1$ & $54 \cdot 9$ & $37 \cdot 8$ & $50 \cdot 7$ & 250 \\
\hline $69 \cdot 0$ & $198 \cdot 2$ & $69 \cdot 2$ & $15+8$ & 693 & $136 \cdot 7$ & 600 & $97 \cdot 8$ & $69 \cdot 3$ & $41 \cdot 1$ & $69 \cdot 3$ & $31 \cdot 1$ \\
\hline \multicolumn{4}{|c|}{$\mathrm{H}^{\prime}=50.07$} & \multicolumn{4}{|c|}{$\mathrm{H}^{\prime}=99 \cdot 7}$. & \multicolumn{4}{|c|}{$H^{\prime}=16 \delta^{\prime} 7$} \\
\hline$\tau$. & $\delta \mathrm{I}_{i}$ & $\tau$ & $\hat{o} \mathrm{~T}_{c}$ & $\tau$. & $\delta \mathrm{I}_{i}$ & $\tau$. & $\delta \mathrm{I}_{c}$ & $r$. & $\delta \mathrm{I}_{i}$ & $\tau$. & $\delta \mathrm{I}_{\sigma^{\prime}}$ \\
\hline $11 \cdot 8^{\prime}$ & $+7 \cdot 3$ & $10^{\prime} 1^{\prime}$ & $+3 \cdot 0$ & $9 \cdot 8^{\prime}$ & $+2 \cdot 4$ & $15^{\cdot} 4^{\prime}$ & 0 & $9 \cdot y^{\prime}$ & +1.2 & $11 \cdot 7^{\prime}$ & +06 \\
\hline $28 \cdot 8$ & $-3 \cdot 4$ & $26 \cdot 8$ & $-7 \cdot 6$ & $25 \cdot 8$ & -66 & $32 \cdot 6$ & -125 & $38 \cdot 5$ & $-12 \cdot 2$ & 257 & $-5 \cdot 2$ \\
\hline $46 \cdot 4$ & -76 & 450 & $-12 \cdot 2$ & 43.9 & $-18 \cdot 7$ & $47 \cdot 7$ & -220 & 580 & $-21 \cdot 4$ & 420 & $-14 \cdot 8$ \\
\hline \multirow[t]{7}{*}{68.9} & $-10 \cdot 8$ & $69 \cdot 3$ & $-157^{\prime}$ & 694 & $-30 \cdot 7$ & 603 & $-31 \cdot 8$ & $69 \cdot 3$ & $-20 \cdot 4$ & $69 \cdot 3$ & -301 \\
\hline & & \multicolumn{4}{|c|}{$\mathrm{H}^{\prime}=241 \cdot 4$} & \multicolumn{4}{|c|}{$\mathrm{H}^{\prime}=3 \overline{5} 8 \cdot 0$} & & \\
\hline & & $\tau$ & $\delta \mathrm{I}_{i}$ & T. & $\delta \mathrm{I}_{c^{\prime}}$ & $\pi$ & $\delta \mathrm{T}_{i}$ & $\tau$. & $\delta \mathrm{I}_{c}$ & & \\
\hline & & $13 \cdot 1^{\prime}$ & -03 & $14 \cdot 2^{\prime}$ & 0.0 & $18 \cdot 8^{\prime}$ & 0.0 & $14 \cdot 2^{\prime}$ & 0.0 & & \\
\hline & & $28 \cdot 9$ & -4.9 & $28 \cdot 9$ & -46 & 38.7 & -43 & $22 \cdot 6$ & $-2 \cdot 3$ & & \\
\hline & & 466 & -133 & $48 \cdot 0$ & $-13 \cdot 6$ & 53.5 & $-10 \cdot 4$ & 520 & -96 & & \\
\hline & & $69 \cdot 3$ & $-25 \cdot 6$ & $69 \cdot 3$ & $-19 \cdot 3$ & 69.3 & -142 & 69.3 & $-15 \cdot 8$ & & \\
\hline
\end{tabular}


$\mathrm{T}=6286 \mathrm{gr} . / \mathrm{mm}^{2}{ }^{2} ; t=14^{\circ} \cdot 7 \mathrm{C}$.

\begin{tabular}{|c|c|c|c|c|c|c|c|c|c|c|c|}
\hline \multicolumn{4}{|c|}{$\mathrm{H}^{\prime}=\mathbf{2} 38$} & \multicolumn{4}{|c|}{$\mathrm{H}^{\prime}=399$} & \multicolumn{4}{|c|}{$\mathrm{H}^{\prime}=10 \cdot 64$} \\
\hline$\tau$ & $\delta \mathrm{I}_{i}$ & $\tau$. & $\delta \mathrm{I}_{c}$ & $\tau$. & $\delta \mathrm{I}_{i}$ & $\tau$ & $\delta \mathrm{I}_{\boldsymbol{c}}$ & $\tau$. & $\delta \mathrm{I}_{i}$ & $\tau$. & $\delta \mathrm{I}_{c^{*}}$ \\
\hline $9 \cdot 3$ & $1 \cdot 8$ & $38^{\prime}$ & $15 \cdot 4$ & $10 \cdot 1^{\prime}$ & $13 \cdot 9$ & $11 \cdot 6^{\prime}$ & $24 \cdot 5$ & $7 \cdot 1^{\prime}$ & $16 \cdot 9$ & $9 \cdot 9^{\prime}$ & $19 \cdot 4$ \\
\hline $24 \cdot 0$ & $5 \cdot 5$ & $29 \cdot 8$ & $43 \cdot 0$ & $24 \cdot 8$ & $36 \cdot 3$ & $26-1$ & $55 \cdot 0$ & $20 \cdot 4$ & $42 \cdot 8$ & $26 \cdot 8$ & 53.5 \\
\hline $38 \cdot 1$ & 113 & $45 \cdot 4$ & $66 \cdot 2$ & $39 \cdot 8$ & $61 \cdot 9$ & $41 \cdot 7$ & $88 \cdot 8$ & $33 \cdot 8$ & $73 \cdot 8$ & $42 \cdot 3$ & $91 \cdot 8$ \\
\hline $51 \cdot 3$ & $20 \bar{z}$ & $\ldots$ & $\ldots$ & $58 \cdot 2$ & $95 \cdot 3$ & $50 \cdot 6$ & $107 \cdot 2$ & $51 \cdot 3$ & 116.5 & $57 \cdot 0$ & $121^{\circ} 0$ \\
\hline $68 \cdot 8$ & $37 \cdot 3$ & 688 & $100 \cdot 8$ & $68 \cdot 8$ & $116 \cdot 4$ & 688 & $142 \cdot 4$ & $68 \cdot 8$ & 1525 & $68 \cdot 8$ & $141 \cdot 5$ \\
\hline \multicolumn{4}{|c|}{$\mathbf{H}^{\prime}=\mathbf{2 4 . 0 3}$} & \multicolumn{4}{|c|}{$\mathrm{H}^{\prime}=49.02}$. & \multicolumn{4}{|c|}{$\mathrm{H}^{\prime}=94 \cdot 9$} \\
\hline$\tau$. & $\delta \mathrm{I}_{i}$ & $\tau$. & $\delta \mathrm{I}_{e}$ & $\tau$. & $\delta \mathrm{I}_{i}$ & $\tau$. & $\delta_{c}$. & $r$. & $\delta \mathrm{I}_{i}$ & $\tau$. & $\delta \mathrm{I}_{c^{*}}$ \\
\hline $6 \cdot 6^{\prime}$ & $7 \cdot 9$ & $9 \cdot 3^{\prime}$ & $10 \cdot 4$ & $10 \cdot 9^{\prime}$ & 47 & $11 \cdot 2^{\prime}$ & $3 \cdot 0$ & $8 \cdot 6^{\prime}$ & 3.5 & $14 \cdot 4^{\prime}$ & 1.5 \\
\hline $23 \cdot 8$ & $36 \cdot 7$ & $26 \cdot 9$ & $40 \cdot 8$ & $23 \cdot 5$ & $20 \cdot 7$ & $27 \cdot 3$ & $21 \cdot 4$ & $22 \cdot 9$ & $4 \cdot 7$ & 31.8 & 26 \\
\hline $36 \cdot 1$ & 637 & $44 \cdot 1$ & $75 \cdot 9$ & $40 \cdot 3$ & 420 & $45 \cdot 4$ & $44 \cdot 3$ & $38 \cdot 7$ & $6 \cdot 3$ & $\ldots$ & ... \\
\hline $55 \cdot 4$ & $100 \cdot 8$ & 563 & $97 \cdot 6$ & 570 & 617 & $\ldots$ & $\ldots$ & 537 & 87 & 503 & $5 \cdot 3$ \\
\hline $68 \cdot 8$ & $120 \cdot 4$ & $68 \cdot 8$ & 115.5 & 68.8 & $72 \cdot 9$ & $68 \cdot 8$ & $67 \cdot 7$ & 68.8 & $10 \cdot 4$ & 68.8 & 79 \\
\hline \multicolumn{4}{|c|}{$\mathrm{H}^{\prime}=168 \cdot 7$} & \multicolumn{4}{|c|}{$\mathrm{H}^{\prime}=254 \cdot 0$} & \multicolumn{4}{|c|}{$\mathrm{H}^{\prime}=351 \cdot 2}$. \\
\hline$\pi$ & $\delta \mathbf{I}_{i}$ & t. & $\delta \mathrm{I}_{c}$ & $r$. & $\delta \mathbf{I}_{i}$ & $\tau$. & $\delta \mathrm{I}_{c}$ & $\tau$. & $\delta \mathbf{I}_{i}$. & $\tau$. & $\delta \mathrm{I}_{c^{*}}$ \\
\hline $9 \cdot 9^{\prime}$ & $+2 \cdot 1$ & $10 \bar{s}^{\prime}$ & +0.9 & $7 \cdot 8^{\prime}$ & $+1 \cdot 1$ & $99^{\prime}$ & +0.9 & 117 & +0.5 & $17 \cdot 0^{\prime}$ & -05 \\
\hline $23 \cdot 2$ & -5.9 & $29 \cdot 2$ & -9.4 & $28 \cdot 9$ & -6.1 & $28 \cdot \overline{5}$ & $-5 \cdot 6$ & 29.8 & -40 & $34 \cdot 6$ & $-6 . \pm$ \\
\hline $43 \cdot 5$ & $-15 \cdot 4$ & 503 & $-19 \cdot 3$ & $45 \cdot 4$ & $-14 \cdot 9$ & ... & $\ldots$ & $49 \cdot 6$ & $-12 \cdot 1$ & 48.7 & $-13 \cdot 1$ \\
\hline $68 \cdot 8$ & -258 & 68.8 & $-26 \cdot 0$ & $68 \cdot 8$ & $-26 \cdot 4$ & $68 \cdot 3$ & $--28 \cdot 1$ & $68 \cdot 8$ & $-20 \cdot 9$ & $68 \cdot 8$ & $-22 \cdot 2$ \\
\hline
\end{tabular}

The effect of the graduated application of twist does not sensibly differ from that obtained by applying the maximum twist at once.

(d) Magnetization under Constant Twist combined with Tensions : $(\mathrm{I}, \mathrm{H})_{\tau, \mathrm{T}}$.

The effect of a constant twist on magnetization is to increase the magnetization in weak fields and to diminish it G 2 
84 Messrs. K. Honda and T. Terada on the

in strong fields. As for curves $(\delta \mathrm{I}, \mathrm{H})_{\tau, \mathrm{T}}$ (dotted lines in figs, $2\left(0\right.$ and 21 ) deduced from $(\mathrm{I}, \mathrm{H})_{\tau, \mathrm{T}}$, the general course is quite similar to that of the curves obtained from $\left(\delta \mathrm{I}_{i}, \tau\right)_{\mathrm{H}, \mathrm{r}}$; but quantitatively there is some difference between these two. The difference, however, becomes less with increased tension.

Nickel.

$\mathrm{T}=1197 \mathrm{gr} . / \mathrm{mm} .^{2} ; t=13^{\circ} \cdot 7 \mathrm{C}$.

\begin{tabular}{|c|c|c|c|c|c|c|c|}
\hline \multicolumn{2}{|c|}{$\tau=0}$. & \multicolumn{2}{|c|}{$\tau=11^{\prime} \cdot 4$} & \multicolumn{2}{|c|}{$\tau=34^{\prime} \cdot 5$} & \multicolumn{2}{|c|}{$\tau=67^{\prime} \cdot 0$} \\
\hline H. & I. & H. & I. & $\mathrm{H}$. & I. & H. & I. \\
\hline 0.87 & $6 \cdot 5$ & 0.81 & $6 \cdot 1$ & 0.79 & $4 \% 3$ & 0.79 & 50 \\
\hline $1 \cdot 88$ & 570 & 178 & $47 \cdot 4$ & $2 \cdot 00$ & $80 \cdot 5$ & $1 \cdot 34$ & 68.8 \\
\hline $4 \cdot 71$ & $151 \% 7$ & $3 \cdot 07$ & $102 \cdot 0$ & $3 \cdot 40$ & $135 \%$ & 1.98 & $133 \cdot 2$ \\
\hline $7 \cdot 68$ & 1960 & 4.99 & $147 \cdot 1$ & $5 \cdot 32$ & $211 \cdot 7$ & 4.04 & $262 \cdot 6$ \\
\hline 10.05 & 2220 & $8 \cdot 11$ & 1953 & 8.36 & 2737 & 8.44 & $310 \cdot 5$ \\
\hline $14 \cdot 49$ & $262 \cdot 1$ & $12 \cdot 48$ & 248.5 & 11.95 & $302 \cdot 6$ & 1361 & 328.8 \\
\hline 35.74 & $371 \cdot 0$ & $34 \cdot 12$ & $360 \cdot 9$ & $33 \cdot 79$ & $368 \cdot 4$ & 33.80 & $366 \cdot 2$ \\
\hline $60 \cdot 4$ & $429 \cdot 9$ & $52 \cdot 7$ & 4061 & $52 \cdot 85$ & $398 \cdot 7$ & $52 \cdot 68$ & 3877 \\
\hline $125 \cdot 1$ & 4840 & $10+\cdot 1$ & 4635 & $105 \cdot 3$ & 4487 & $10+6$ & 4257 \\
\hline $182 \cdot 2$ & 5027 & $169 \cdot 1$ & 491.0 & $171^{\circ} 0$ & 4787 & $169 \cdot 3$ & $454 \cdot 2$ \\
\hline $218 \cdot 4$ & $509 \cdot 2$ & $220 \cdot 4$ & 5016 & 2225 & $491 \cdot 1$ & $220 \cdot 4$ & $468 \cdot \overline{0}$ \\
\hline 3044 & 5186 & $289 \cdot 9$ & $503 \cdot 1$ & $282 \cdot 8$ & $500 \cdot 4$ & $279 \cdot 7$ & $479 \cdot 1$ \\
\hline $369 \cdot 1$ & 5220 & 35699 & 5127 & $363 \cdot 2$ & $507 \cdot 9$ & $357 \cdot 3$ & $489 \cdot 1$ \\
\hline
\end{tabular}

$\mathrm{T}=3546 \mathrm{gr} . / \mathrm{mm} .^{2}: t=13^{\circ} \cdot 7 \mathrm{C}$.

\begin{tabular}{|c|c|c|c|c|c|c|c|}
\hline \multicolumn{2}{|c|}{$\tau=0}$. & \multicolumn{2}{|c|}{$r=11^{\prime} \cdot 9}$. & \multicolumn{2}{|c|}{$\tau=34^{\prime} \cdot 6$. } & \multicolumn{2}{|c|}{$\tau=66^{\circ} \cdot 9$} \\
\hline H. & I. & H. & I. & H. & I. & H. & I. \\
\hline 0.80 & $4 \cdot 8$ & 0 & $4 \cdot 1$ & 0.80 & 5 & 0.79 & $2 \cdot 7$ \\
\hline & 13 & & & & & 75 & 148 \\
\hline 3 & 29 & 3 & 2 & & $24 \cdot 6$ & $2 \cdot 84$ & $106 \cdot 4$ \\
\hline 6.55 & 5 & & 3 & & & 6 & $91 \cdot \tilde{0}$ \\
\hline $\mathbf{9} 73$ & 91.5 & & & & 123.0 & 6.52 & 248.5 \\
\hline $15 \cdot 03$ & $136^{\circ} 4$ & $11 \cdot 67$ & $107 \cdot 3$ & $12 \cdot 08$ & 10 & $11 \cdot 22$ & $277 \cdot 4$ \\
\hline $23 \cdot 30$ & $191^{\circ} 4$ & 17.5 & 15 & $22 \cdot 53$ & 250 & & \\
\hline $\begin{array}{l}33.86 \\
54 \cdot 1\end{array}$ & $\begin{array}{l}2450 \\
321 \cdot 3\end{array}$ & $\begin{array}{l}33.48 \\
57.28\end{array}$ & $\begin{array}{l}249 \cdot 7 \\
322 \cdot 6\end{array}$ & $\begin{array}{l}33 \cdot 68 \\
51 \cdot 8.5\end{array}$ & $\begin{array}{l}38.2 \\
38.2\end{array}$ & $\begin{array}{l}33 \cdot 15 \\
52 \cdot 87\end{array}$ & $\begin{array}{l}3338.9 \\
3673\end{array}$ \\
\hline $\begin{array}{r}113 \cdot 6 \\
113\end{array}$ & $430 \cdot 2$ & $101 \cdot 8$ & 414.9 & 113.3 & 418.8 & $\begin{array}{l}112 \% 8 \\
112 \%\end{array}$ & $\begin{array}{l}300 \\
425 \cdot 6\end{array}$ \\
\hline & 462.6 & $162 * 3$ & 458 & 171.0 & 454.3 & 169 & 456.5 \\
\hline & 48 & & 48 & & & & $482 \cdot 2$ \\
\hline & $499^{\circ}$ & $356 \cdot 9$ & 49 & $35 i$ & $493 \cdot 1$ & 35 & 500 \\
\hline
\end{tabular}


$\mathrm{T}=6286 \mathrm{gr} . / \mathrm{mm}^{2} ; t=13^{\circ} \cdot 7 \mathrm{C}$.

\begin{tabular}{|c|c|c|c|c|c|c|c|}
\hline \multicolumn{2}{|c|}{$\tau=0}$. & \multicolumn{2}{|c|}{$\tau=11^{\prime} \cdot 8$} & \multicolumn{2}{|c|}{$\tau=34^{\prime} \cdot 6$} & \multicolumn{2}{|c|}{$\tau=69^{\prime} \cdot 0$} \\
\hline H. & I. & H. & I. & $\mathrm{H}$. & I. & H. & I. \\
\hline $1 \cdot 46$ & $5 \cdot 6$ & $1 \cdot 17$ & $3 \cdot 8$ & $1: 39$ & $4 \cdot 0$ & $0 \cdot 88$ & $2 \cdot 6$ \\
\hline 303 & $11 \%$ & $2 \cdot 53$ & $8 \cdot 4$ & $3 \cdot 12$ & 114 & $3 \cdot 14$ & $25 \cdot 5$ \\
\hline $5 \cdot 04$ & $21 \cdot 9$ & 4.26 & $16 \cdot 0$ & $4 \cdot 89$ & $21 \cdot 7$ & $5 \cdot 40$ & $158 \cdot 4$ \\
\hline 8.92 & $43 \cdot 1$ & $9 \cdot 89$ & $43 \cdot 9$ & $7 \cdot 80$ & $58 \cdot 8$ & $8 \cdot 16$ & 211.9 \\
\hline $14 \cdot 29$ & $72 \cdot 3$ & $15 \cdot 38$ & $74: 3$ & $11 \cdot 58$ & $119 \cdot 7$ & $13 \cdot 27$ & 2320 \\
\hline $23 \cdot 67$ & $114 \cdot 8$ & $23 \cdot 86$ & $119 \overline{5}$ & $23 \cdot 35$ & 1759 & $23 \cdot 77$ & 2603 \\
\hline $34 \cdot 77$ & $156^{-8}$ & $34 \cdot 93$ & $162 \cdot 2$ & $34 \cdot 98$ & 2110 & 35.36 & $285 \cdot 7$ \\
\hline $57 \cdot 33$ & 2297 & $56 \cdot 8$ & $234 \cdot 7$ & $56 \cdot 2$ & $268 \cdot 9$ & 56.58 & $325 \cdot 7$ \\
\hline $98 \cdot 6$ & $341 \cdot 0$ & $88 \cdot 8$ & $321 \cdot 6$ & $88 \cdot 1$ & $338 \cdot 5$ & $88 \cdot 1$ & $372 \cdot 5$ \\
\hline $161 \cdot 7$ & $429 \cdot 0$ & $134: 3$ & $396: 3$ & $14^{\prime} \cdot 6$ & $412 \cdot 3$ & $147 \cdot 3$ & $427 \cdot 6$ \\
\hline $195 \cdot 5$ & $451 \cdot 2$ & 1904 & $441 \cdot 9$ & $196 \cdot 3$ & $44 \pm \cdot 6$ & $196 \cdot 2$ & $445 \cdot 2$ \\
\hline 2727 & $478 \cdot 7$ & $270 \cdot 2$ & 4715 & $267 \cdot 5$ & $471 \cdot 4$ & $267 \cdot 5$ & $481 \cdot 3$ \\
\hline $381 \cdot 7$ & $465 \cdot 7$ & $380 \cdot 5$ & $489 \cdot 8$ & $375 \cdot 4$ & $491 \cdot 3$ & $373 \cdot 0$ & $503 \cdot 8$ \\
\hline
\end{tabular}

It is to be noticed that in nickel, the initial and the cyclic effects of tension or twist on magnetization nearly coincide with each other except in weak fields, and that the change of magnetization does not much depend on the order of magnetizing and straining. Thus, in nickel, the hysteresis effect is comparatively small except in weak fields; and therefore the agreement between the theory regarding magnetostriction and the experiment might well have been expected. Thus, in our previous investigation, we found that the changes of the modulus of elasticity by magnetization for different orders of magnetizing and straining fairly coincided with each other, while in the case of rigidity, the difference was somewhat greater. In the present investigation also, the tension effect shows a better agreement for different orders of magnetizing and straining than for the torsion effect.

\section{NICKEI-ST'EELS containing $28 \cdot 74,50 \cdot 72$, and 70.32 per cent. of Nickel.}

As for nickel-steels, experiments on the effect of stress on magnetization have been very few. So far as we know, the effect of tension only was studied by $\mathrm{H}$. Tomlinson * with

* Tomlinson, Proc. Roy. Soc. lvi. p. 103 (1894) ; Beibl. xviii. p. 952 2. 
nickel-steels of 22,25 , and 30 per cent. of nickel, and by Prof. H. Nagaoka and one of us ${ }^{*}$ with nickel-steels of 35 and 45 per cent. of nickel. Hence somewhat detailed descriptions of the phenomena will not be unnecessary.

\section{(a) Change of Magnetization by Tension under Constant Field : $(\delta \mathrm{I}, \mathrm{T})_{\mathrm{H}}$.}

The magnetization increases at first rather rapidly, but afterward slowly with the tension. The increase in low fields is tolerably large, but in strong fields it is very small. The initial effect is significant only for weak fields, where the cyclic is remarkably less than the initial. The following tables and figs. 22, 23, \& 24 (Pl. III.) show these changes of magnetization.

Curves $\left(\delta \mathrm{I}_{i}, \mathrm{H}\right)_{\mathrm{T}}$ from $\left(\delta \mathrm{I}_{i}, \mathrm{~T}\right)_{\mathrm{H}}$ rise rapidly with the field, attain sharp maxima at low fields, fall at first rapidly and then gradually to asymptotic values, as shown in figs. 25, 26, and 27 in full lines. The maximum of $\delta I$ increases with tension. For the same tension, the maximum rapidly increases with the percentage content of nickel.

28.74 per cent. Nickel-Steel.

Initial $\mathrm{T}=134 \mathrm{gr} . / \mathrm{mm}^{2} ; t=14^{\circ} \cdot 0 \mathrm{C}$.

\begin{tabular}{|c|c|c|c|c|c|c|c|c|}
\hline \multirow{2}{*}{$\mathrm{H}^{\prime}$. } & \multicolumn{2}{|c|}{$\mathrm{T}=1370 \mathrm{gr} . / \mathrm{mm} .^{2}$} & \multicolumn{2}{|c|}{$\mathrm{T}=2706 \mathrm{gr}}$. & \multicolumn{2}{|c|}{$\mathrm{T}=4077 \mathrm{gr}}$. & \multicolumn{2}{|c|}{$\mathrm{T}=6818 \mathrm{gr}}$. \\
\hline & $\delta \mathrm{I}_{i}$ & $\delta \mathrm{I}_{c}$ & $\delta \mathrm{I}_{i}$ & $\delta \mathrm{I}_{c}$ & $\hat{\delta} \mathbf{T}_{i}$ & $\delta \mathrm{I}_{c^{*}}$ & $\delta \mathbf{I}_{\boldsymbol{i}}$ & $\delta \mathrm{I}_{c^{*}}$ \\
\hline 0.11 & $I \cdot 1$ & 8.8 & $\ldots$ & $\ldots$ & $22 \cdot 2$ & $30 \cdot 3$ & $36 \cdot 0$ & $35 \cdot 3$ \\
\hline 0.25 & $20 \cdot 1$ & $26 \cdot 4$ & $62 \cdot 4$ & 57.5 & $97 \cdot 5$ & $77 \cdot 6$ & $130 \cdot 3$ & $94 \cdot 1$ \\
\hline $0 \cdot 49$ & $46 \cdot 7$ & $31 \cdot 8$ & $97 \cdot 6$ & 647 & $129 \cdot 2$ & $86 \cdot 1$ & 155.5 & $104 \cdot 0$ \\
\hline $1 \cdot 11$ & 43.4 & $31 \cdot 7$ & $78 \cdot 0$ & $59 \cdot 9$ & $97 \cdot 6$ & 76.4 & $113 \cdot 0$ & 90.0 \\
\hline $2 \cdot 30$ & $31 \cdot 6$ & $26 \cdot 8$ & $52 \cdot 2$ & $45 \cdot 7$ & $63 \cdot 3$ & $56 \cdot 1$ & $72 \cdot 2$ & 64.5 \\
\hline 465 & 18.8 & $17 \cdot 2$ & $28 \cdot 9$ & $27 \cdot 0$ & $34 \cdot 0$ & 320 & $38 \cdot 6$ & $36 \cdot 3$ \\
\hline $10 \cdot 68$ & 59 & $5 \cdot 3$ & $\ldots$ & $\ldots$ & 11.0 & $10 \cdot 0$ & $13 \cdot 8$ & $12 \cdot 8$ \\
\hline $24 \cdot 32$ & 23 & 20 & $\ldots$ & $\ldots$ & $5 \cdot 9$ & $5 \cdot 3$ & 8.8 & $8 \cdot 0$ \\
\hline $55 \cdot 48$ & $1 \cdot 5$ & $1 \cdot 6$ & $\ldots$ & $\ldots$ & $4 \cdot 9$ & 4.9 & 7.8 & $8 \cdot 0$ \\
\hline $172 \cdot 9$ & 1.5 & 13 & $\ldots$ & $\cdots$ & $4 \cdot 4$ & $4 \cdot 1$ & $7 \cdot 4$ & $7 \cdot 0$ \\
\hline $374 \cdot 3$ & $1 \cdot 4$ & $1 \cdot 3$ & $\ldots$ & $\cdots$ & 40 & 39 & $6 \cdot 6$ & $6 \cdot 6$ \\
\hline
\end{tabular}

* Nagaclia and Honda, Jour. Coll. Sci. xvi. Art. 8 (1902). 
$50 \cdot 72$ per cent. Nickel-Steel.

Initial $\mathrm{T}=160 \mathrm{gr} . / \mathrm{mm}^{2} ; t=13^{\circ} \cdot 7 \mathrm{C}$.

\begin{tabular}{|c|c|c|c|c|c|c|c|c|c|c|}
\hline \multirow{2}{*}{$\mathbf{H}^{\prime}$. } & \multicolumn{2}{|c|}{$\mathrm{T}=1645 \mathrm{gr} / \mathrm{mm}$} & \multicolumn{2}{|c|}{$\mathrm{T}=3249 \mathrm{gr}$} & \multicolumn{2}{|c|}{$\mathrm{T}=4894 \mathrm{gr}$} & \multicolumn{2}{|c|}{$\mathrm{T}=6540 \mathrm{gr}}$. & \multicolumn{2}{|c|}{$\mathrm{T}=818 \tilde{\mathrm{gr}}}$. \\
\hline & $\delta \mathrm{I}_{i}$ & $\delta \mathrm{I}_{c}$ & $\delta \mathrm{I}_{i}$ & $\delta \mathrm{I}_{e}$ & $\partial \mathrm{I}_{i}$ & $\delta \mathrm{I}_{c^{*}}$ & $\delta \mathrm{I}_{i}$ & $\delta \mathrm{I}_{c^{*}}$ & $\delta \mathrm{T}_{i}$ & $\delta \mathrm{I}_{c^{*}}$ \\
\hline 0.29 & 1083 & 91.0 & $222 \cdot 6$ & 1388 & 277.8 & $153 \cdot 2$ & $300 \cdot 3$ & 155.1 & 3110 & 1537 \\
\hline $0 \cdot 70$ & 2667 & $140 \cdot 5$ & 437.9 & $212 \cdot 1$ & 5039 & 2333 & $528 \cdot 3$ & $235 \cdot 1$ & 5397 & 2320 \\
\hline $1 \cdot 27$ & 319.8 & $157 \cdot 3$ & 4763 & 2334 & 534.8 & 254.9 & $552 \cdot 3$ & 255.9 & $557 \cdot 1$ & 2500 \\
\hline 1.95 & $\geq 53 \cdot 1$ & $148 \cdot 7$ & $3.79 \cdot 2$ & 2154 & 3920 & 2303 & 397.7 & $227 \cdot 5$ & 3902 & $219 \cdot 4$ \\
\hline 276 & 1966 & 1368 & 276.3 & 193.9 & 2987 & $207 \cdot 3$ & 2998 & $204 \cdot 1$ & 394.0 & 195.2 \\
\hline 552 & 134.5 & 1088 & $186^{\circ} 4$ & $151 \%$ & 2025 & 1625 & 2040 & $161 \cdot 1$ & $199 \cdot 1$ & $154 \cdot 4$ \\
\hline 10.71 & $84 \cdot 4$ & $75 \cdot 4$ & 113.8 & 1020 & 1225 & $109 \cdot 4$ & $123 \cdot$ & $109 \cdot 4$ & 1205 & 1060 \\
\hline $23 \cdot 77$ & 34.2 & 32.8 & 46.1 & 43.5 & $49 \cdot 4$ & $4 i \div 6$ & $\ldots$ & $46 \cdot 6$ & 47.9 & 45.0 \\
\hline $51 \cdot 44$ & 7.9 & $7 \cdot 7$ & $10 \cdot 7$ & 105 & $11 \cdot 7$ & 11.0 & $\ldots$ & $\ldots$ & $10 \cdot 2$ & $9 \cdot 2$ \\
\hline $151 \cdot 4$ & 0.7 & 0.4 & 0.9 & $0 \cdot 4$ & 0.9 & 04 & $\ldots$ & $\ldots$ & 0.2 & 0.0 \\
\hline 360.0 & 0.0 & 0.0 & 0.0 & 0.0 & 00 & 0.0 & $\ldots$ & $\ldots$ & 00 & 00 \\
\hline
\end{tabular}

70.32 per cent. Nickel-Steel.

Initial $\mathrm{T}=1 う 6 \mathrm{gr} . / \mathrm{mm}^{2} ; t=14^{\circ} \cdot 5 \mathrm{C}$.

\begin{tabular}{|c|c|c|c|c|c|c|c|c|c|c|}
\hline \multirow{2}{*}{$\mathrm{H}^{\prime}$. } & \multicolumn{2}{|c|}{$\mathrm{T}=1604 \mathrm{gr} . / \mathrm{mm}^{2}$} & \multicolumn{2}{|c|}{$\mathrm{T}=3170 \mathrm{gr}}$. & \multicolumn{2}{|c|}{$\mathrm{T}=4774 \mathrm{gr}$} & \multicolumn{2}{|c|}{$\mathrm{T}=6379 \mathrm{gr}}$. & \multicolumn{2}{|c|}{$\mathrm{T}=7984 \mathrm{gr}$} \\
\hline & $\delta \mathrm{I}_{i}$ & $\delta \mathrm{I}_{c}$ & $\delta \mathrm{I}_{\dot{b}}$ & $\delta \mathrm{I}_{c}$ & $\partial \mathrm{I}_{i}$ & $\delta \mathrm{I}_{e}$ & $\delta \mathbf{I}_{i}$ & $\delta \mathrm{I}_{e}$ & $\delta I_{i}$ & $\delta \mathbf{I}_{c^{*}}$ \\
\hline $0 \cdot 13$ & 257 & $32 \cdot 5$ & $90 \cdot 0$ & $58 \cdot 1$ & 1113 & $65 \cdot 1$ & $107 \cdot 0$ & 66.3 & 94.5 & $67 \cdot 2$ \\
\hline 0.29 & $44 \cdot 4$ & $44 \cdot 5$ & $94: 5$ & & 1096 & $85 \cdot 2$ & $119 \cdot 2$ & $\ldots$ & $13 \% 4$ & 913 \\
\hline 0.70 & $182 \cdot 4$ & $143 \cdot 5$ & $473 \cdot 3$ & 2250 & $58 \div-4$ & $296 \cdot 3$ & $635 \cdot 1$ & $\begin{array}{l}\ldots \\
\ldots\end{array}$ & $665 \cdot 1$ & $339 \cdot 1$ \\
\hline 1.08 & $342 \cdot 3$ & 152.0 & 586.5 & $260 \cdot 4$ & $666^{2}$ & 316.6 & $703 \cdot 5$ & $\cdots$ & $728 \cdot 0$ & 364.7 \\
\hline 1.49 & $321 \cdot 4$ & $1+9 \cdot 2$ & $482 \cdot 5$ & $2+60$ & 546.0 & $298 \cdot 3$ & 578.8 & $\ldots$ & $600 \%$ & $344 \cdot 6$ \\
\hline 266 & $198 \cdot 1$ & $131 \cdot 6$ & 301.5 & $208 \cdot 6$ & $352 \cdot 2$ & $249 \cdot 8$ & $379 \cdot 2$ & $\ldots$ & $381 \cdot 6$ & $287 \cdot 8$ \\
\hline 5.05 & 1133 & 932 & $168^{\circ} 5$ & $142 \cdot 7$ & $199 \cdot 8$ & $171 \cdot 1$ & $\ldots$ & $\ldots$ & $228 \cdot 2$ & $198^{\circ}$ \\
\hline 10.69 & $46 \cdot 9$ & $4 \cdot 2 \cdot 5$ & $70 \cdot 7$ & 657 & 857 & 80.2 & $\ldots$ & $\begin{array}{l}\cdots \\
\ldots\end{array}$ & $101 \cdot 7$ & 95.7 \\
\hline $24 \cdot 52$ & $10 \cdot 7$ & $10 \cdot 3$ & $17 \cdot 5$ & 175 & 238 & $2 \cdot 3$ & $\ldots$ & ... & $28 \cdot 4$ & 28.5 \\
\hline $49 \cdot 70$ & 1.8 & 20 & 1,0 & $\ldots$ & 47 & $4 \cdot 5$ & $\ldots$ & ... & 6.4 & $6 \cdot 2$ \\
\hline $129 \cdot 3$ & $0 \cdot 4$ & $\ldots$ & $\ldots$ & $\ldots$ & $0 . \overline{7}$ & $\ldots$ & $\ldots$ & $\ldots$ & 0.8 & $\ldots$ \\
\hline 22 & $0 \cdot 0$ & $\ldots$ & $\ldots$ & 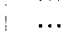 & $0 \cdot 1)$ & $\ldots$ & $\ldots$ & $\ldots$ & $0 \cdot 1$ & $\ldots$ \\
\hline $393 \cdot 2$ & 0.0 & $\ldots$ & $\ldots$ & ... & $0-0$ & ... & $\ldots$ & $\ldots$ & 0.0 & $\ldots$ \\
\hline
\end{tabular}

The effect of applying the maximum tension at once does not materially differ from that obtained by graduated application of it.

The change of magnetization by tension under different initial loadings nearly coincides with the above result in the case of 28.74 per cent. $\mathrm{Ni}$; but in 50.72 per cent. $\mathrm{Ni}$ and 70.32 per cent. $\mathrm{Ni}$ the change is generally greater in the present case than in the former. 
(b) Magnetization under Constant Tensions : $(\mathrm{I}, \mathrm{H})_{\Gamma}$.

Among other ferromagnetic metals and alloys, nickel-steels are characterized by the extraordinary steepness of the curve of magnetization; in a field of 5 c.G.s. units, the magnetization attains a value which is only a little short of its saturation value. The steepness increases with tension, first rapidly and then gradually to an asymptotic value. The enormous values of susceptibility $\kappa$ are given in the following table and plotted in fig. 28. It will be noticed that the maximum value increases with the percentage of nickel. In 70.32 per cent. $\mathrm{Ni}$, the susceptibility even attains a maximum value of 1015 for $T=4930 \mathrm{gr} . / \mathrm{mm}^{2}$, which is several times as great as the maximum susceptibility of a well annealed Swedish iron. In very weak fields, the magnetization is considerably increased by tension, but in higher fields comparatively little.

\begin{tabular}{|c|c|c|c|c|c|c|}
\hline \multirow{3}{*}{ H. } & \multicolumn{2}{|c|}{2874 per cent. Ni. } & \multicolumn{2}{|c|}{50.72 per cent. $\mathrm{Ni}$. } & \multicolumn{2}{|c|}{ 70.32.per cent. Ni. } \\
\hline & \multicolumn{2}{|c|}{$\kappa}$. & \multicolumn{2}{|c|}{ k. } & \multicolumn{2}{|r|}{$\boldsymbol{\kappa}}$. \\
\hline & $\mathrm{T}=134 \mathrm{gr}$. & $\mathrm{T}=6952 \mathrm{gr}$ & $\mathrm{T}=160 \mathrm{gr}$ & $\mathrm{T}=8344 \mathrm{gr}$ & $\mathrm{T}=156 \mathrm{gr}$. & $\mathrm{T}=4930 \mathrm{gr}$ \\
\hline $0 \cdot 20$ & 75 & 80 & 55 & 10 & 10 & 33 \\
\hline $0 \cdot 30$ & 110 & 390 & 110 & 25 & 17 & 95 \\
\hline $0 \cdot 40$ & 125 & 530 & 153 & 75 & 28 & 200 \\
\hline $0 \cdot 60$ & 145 & 445 & 243 & 380 & $6 t$ & 470 \\
\hline 0.80 & 150 & 367 & 337 & 710 & 120 & 1015 \\
\hline $1 \cdot 00$ & 140 & 297 & 410 & 800 & 230 & 860 \\
\hline $1 \cdot 30$ & 124 & 230 & 467 & 685 & 296 & 677 \\
\hline $1 \cdot 60$ & 113 & 190 & 442 & 593 & 288 & 570 \\
\hline 200 & 99 & 157 & 397 & 503 & 270 & 470 \\
\hline $3 \cdot 00$ & 73 & 107 & 299 & 370 & 220 & 322 \\
\hline $5 \cdot 00$ & 50 & 64 & 202 & 232 & 160 & 196 \\
\hline $7 \cdot 00$ & 36 & 46 & 150 & 170 & 123 & 140 \\
\hline
\end{tabular}

Curves $(\delta \mathrm{I}, \mathrm{H})_{\mathrm{T}}$ (figs. 25, 26, and 27, doited lines) deduced from $(I, H)_{T}$ take a course quite similar to those deduced from $\left(\delta[, T)_{H}\right.$. In $28 \cdot 74$ per cent. Ni the maximum $\delta I$ is generally greater, and the asymptotic value decidedly greater than in $\left(\delta I_{i}, T\right)_{\text {н }}$ for the same tension and field. On the contrary, $\delta \mathrm{I}$ of 50.72 per cent. $\mathrm{Ni}$ is always less than the corresponding value in the last experiment. In $70.32 \mathrm{per}$ cent. $\mathrm{Ni}$ there is a fair coincidence between the two values of $\delta \mathrm{I}$. 
28.74 per cent. Nickel-Steel.

$$
t=14^{0.0}
$$

\begin{tabular}{|c|c|c|c|c|c|c|c|}
\hline \multicolumn{2}{|c|}{$\mathrm{T}=134 \mathrm{gr} . / \mathrm{mm} .^{2}$} & \multicolumn{2}{|c|}{$\mathrm{T}=1504 \mathrm{gr}}$. & \multicolumn{2}{|c|}{$\mathrm{T}=421 \mathrm{gr}}$. & \multicolumn{2}{|c|}{$\mathrm{T}=6952 \mathrm{gr}}$. \\
\hline $\mathbf{H}$. & I. & $\mathrm{H}$. & I. & $\mathrm{H}$. & I. & H. & I. \\
\hline 0.23 & $20 \cdot 8$ & $0 \cdot 22$ & 268 & $0 \cdot 13$ & 7.9 & 0.09 & 38 \\
\hline 072 & $108 \cdot 6$ & 0.73 & $178 \cdot 4$ & 0.24 & 630 & 0.24 & $41 \cdot 6$ \\
\hline 077 & $114 \cdot 0$ & 1.02 & 204.8 & 0.33 & 167.9 & $0 \cdot 30$ & 1189 \\
\hline 1.33 & $162 \cdot 8$ & $1 \cdot 41$ & $225 \cdot 2$ & 0.52 & $243 \cdot 3$ & 0.53 & $260 \cdot 6$ \\
\hline 200 & $192 \cdot 1$ & 2.53 & 257.4 & 0.82 & $272 \cdot 1$ & 0.77 & $280 \cdot 9$ \\
\hline $3 \cdot 26$ & $2 \div 55$ & 363 & $274 \cdot 1$ & 1.21 & $286^{\circ 5}$ & $1 \% 24$ & $295 \cdot 5$ \\
\hline 476 & $249 \cdot 1$ & $7 \cdot 31$ & 2997 & $4 \cdot 08$ & $3 \geq 0 \div 3$ & 336 & 3176 \\
\hline $12 \cdot 11$ & $287 \cdot 1$ & 1283 & 3120 & 11.57 & $339 \cdot 6$ & 11.28 & $338-6$ \\
\hline $19 \cdot 27$ & 2960 & $19 \cdot 12$ & 3180 & 18.96 & 34 & $18 \cdot 96$ & 3456 \\
\hline 24.06 & 2997 & $24 \cdot 44$ & $321 \cdot 4$ & 2431 & $349 \cdot 1$ & 24.32 & $349 \cdot 0$ \\
\hline $50 \cdot 1$ & $308 \cdot 8$ & $50 \cdot 1$ & $330 \cdot 2$ & 4975 & 357.7 & $49 \cdot 9$ & 357.8 \\
\hline $123 \cdot 8$ & $320 \cdot 3$ & 12355 & 341.0 & $122 \cdot 4$ & 368.5 & $122 \cdot 4$ & 3687 \\
\hline $230 \cdot 5$ & $329 \cdot 0$ & $229 \cdot 0$ & $349 \cdot 7$ & $2 \pm 79$ & $377 \% 2$ & $227 \cdot 5$ & $377^{2}$ \\
\hline $374 \cdot 8$ & $337 \cdot 2$ & $372 \cdot 6$ & 3578 & $370 \cdot 2$ & 3852 & 3709 & 3851 \\
\hline
\end{tabular}

$52 \cdot 72$ per cent. Nickel-Steel.

$$
t=13^{\circ} \cdot 1 \mathrm{C} .
$$

\begin{tabular}{|c|c|c|c|c|c|c|c|c|c|}
\hline \multicolumn{2}{|c|}{$\mathrm{T}=983 \mathrm{gr} . / \mathrm{mm}^{2}{ }^{2}$} & \multicolumn{2}{|c|}{$\mathrm{T}=3450 \mathrm{gr}$} & \multicolumn{2}{|c|}{$\mathrm{T}=5054 \mathrm{gr}$} & \multicolumn{2}{|c|}{$\mathrm{T}=6699 \mathrm{gr}$} & \multicolumn{2}{|c|}{$\mathrm{T}=8344 \mathrm{gr}}$. \\
\hline H. & I. & $\mathrm{H}$. & I. & H. & I. & II. & I. & H. & $\mathrm{I}$. \\
\hline 0.30 & .34 .0 & 0.32 & $28 \cdot 6$ & 0.33 & $24 \cdot 7$ & $0 \cdot 31$ & $23 \cdot 3$ & 038 & 154 \\
\hline 0.51 & 965 & 0.48 & 1300 & 0.50 & 160.4 & 0.49 & 1137 & 0.49 & $80 \cdot 6$ \\
\hline 075 & $262 \cdot 6$ & 0.59 & 314.7 & 0.62 & $361 \cdot 1$ & 066 & 458.6 & 0.63 & 285.9 \\
\hline 1.48 & 739 & 0.85 & $597^{\circ}$ & 0.89 & 681 & 1.08 & 839 & 0.80 & 561.8 \\
\hline 173 & 810 & 1.41 & $89 y$ & 1.38 & 928 & 1.64 & 996 & $0 \cdot 90$ & 717 \\
\hline 262 & 949 & $2 \cdot 15$ & 1043 & $2 \cdot 12$ & 1062 & 2.83 & 1118 & 158 & 964 \\
\hline 4.01 & 1028 & 3.83 & 1143 & $4 \cdot 42$ & 1177 & $4 \cdot \hat{\mathrm{\varepsilon}} 8$ & 1180 & 305 & 1106 \\
\hline $\begin{array}{l}4.92 \\
4\end{array}$ & 1064 & 5.49 & 1181 & 6.87 & 1215 & $7 \cdot 57$ & 1224 & $7 \cdot 27$ & 1201 \\
\hline 17.01 & 1210 & 1088 & 1229 & 11.96 & 1244 & 1379 & 1253 & 12.94 & 1232 \\
\hline 2373 & 1235 & $22 \cdot 62$ & 1260 & $22 \cdot 78$ & 1267 & $23 \cdot 17$ & 1271 & 22.95 & 1253 \\
\hline $43 \times 47$ & 1267 & 48.06 & 1279 & $\therefore 4280$ & 1283 & 43.22 & 1287 & $42 \cdot 81$ & 1269 \\
\hline 110.9 & 1290 & 107.0 & 1295 & $305 \cdot 6$ & 1298 & 1053 & 1302 & $105 \cdot 3$ & 1284 \\
\hline $234 \cdot 8$ & 1298 & 228.0 & 1302 & 225.5 & 1305 & $224 \cdot 6$ & 1310 & 226.1 & 1292 \\
\hline $384 \cdot 1$ & 1302 & 371.5 & 1306 & 367.9 & 1309 & $367 \cdot 3$ & 1314 & 3667 & 1296 \\
\hline
\end{tabular}


70.32 per cent. Nickel-Steel.

$$
t=13^{\circ} \cdot 5 \mathrm{C} \text {. }
$$

\begin{tabular}{|c|c|c|c|c|c|c|c|c|c|}
\hline \multicolumn{2}{|c|}{$\mathrm{T}=156 \mathrm{gr} . / \mathrm{mml}^{2}$} & \multicolumn{2}{|c|}{$\mathrm{T}=959 \mathrm{gr}$} & \multicolumn{2}{|c|}{$\mathrm{T}=1761 \mathrm{gr}$} & \multicolumn{2}{|c|}{$\mathrm{T}=3366 \mathrm{gr}$} & \multicolumn{2}{|c|}{$\mathrm{T}=4930 \mathrm{gr}}$. \\
\hline H. & I. & H. & I. & H. & I. & H. & I. & H. & I. \\
\hline $0 \cdot 25$ & $18 \cdot 5$ & 0.23 & 21.9 & 0.31 & $26 \cdot 9$ & 0.26 & 185 & $0 \cdot 24$ & 95 \\
\hline 0.79 & 945 & 0.75 & 128.8 & 0.70 & $162 \cdot 2$ & 0.46 & 1329 & 0.43 & $180 \cdot 6$ \\
\hline 0.86 & 127.9 & 0.90 & 327.5 & 0.85 & $\tilde{5} 57 \cdot \overline{8}$ & 0.57 & 206.5 & 0.60 & $289 \cdot 0$ \\
\hline $1 \cdot 13$ & $325 \%$ & 1.53 & 609 & $1 \cdot 74$ & 758 & $0 \cdot 74$ & 478.8 & $0 \cdot 64$ & $347 \cdot 7$ \\
\hline 1.52 & $430 \cdot 4$ & 1.78 & 654 & 280 & 841 & 0.85 & 738 & 0.71 & 5320 \\
\hline 1.80 & $503 \cdot 6$ & 277 & 763 & $3 \cdot 83$ & $88 t$ & $2 \cdot 20$ & 903 & 0.76 & 734 \\
\hline 4.66 & 769 & $5 \cdot 77$ & 892 & 632 & 937 & $3 \cdot 56$ & 946 & $1 . \pm 5$ & 906 \\
\hline 8.04 & 871 & 1000 & $9 \overline{5} 5$ & $11 \cdot 53$ & 985 & 6.72 & 981 & 3.38 & 970 \\
\hline 11.03 & 927 & 19.28 & 1008 & 19.67 & 1016 & $19 \cdot 26$ & 1026 & 675 & 996 \\
\hline 2654 & 998 & 2466 & 1022 & $25 \cdot 34$ & 1028 & $24 ; 3$ & 1034 & 2402 & 1032 \\
\hline $50 \cdot 9$ & 1022 & $51 \cdot 2$ & 1045 & $52 \cdot 0$ & 1047 & $51 \cdot 1$ & 1049 & $50 \cdot 9$ & 1043 \\
\hline $10+4$ & 1030 & 1032 & 1052 & $104 \cdot 3$ & $105 \overline{5}$ & 102.5 & 10504 & $99 \cdot 0$ & 1048 \\
\hline $204 \cdot 4$ & I053 & $20+\cdot 1$ & 1054 & $202 \cdot 3$ & 1057 & $200 \cdot 6$ & 1056 & 201.0 & 1049 \\
\hline 370.0 & 1034 & $368 \cdot 5$ & 1055 & 3650 & 1057 & $362 \cdot 6$ & 1057 & $365 \cdot 1$ & 1051 \\
\hline
\end{tabular}

(c) Change of Magnetization by Twist under Different Tensions: $(\delta \mathrm{I}, \tau)_{\mathrm{B}, \mathrm{r}}$.

In very low fields the maguetization considerably increases with twist; in higher fields it first increases, but afterward begins to decrease with the twist, and in still higher fields the magnetization decreases nearly uniformly with the twist, as shown in figs. $29,31,33,35,37, \& 38$ (Pl. IV.). The change of magnetization rapidly increases with the percentage content of nickel.

As for the cyclic effect (figs. 30, 32, 34, 36, 37, and 39), it coincides fairly with the initial, except in weak fields. With 28.74 per cent. Ni and 70.32 per cent. $\mathrm{Ni}$, the increase of magnetization is only observable in very weak fields, and the magnetization generally decreases with twist. With 50.72 per cent. $\mathrm{Ni}$, the magnetization first increases with the $t$ wist, attains a maximum, and then decreases. As the tension is increased, the change becomes gradually less.

Curves $\left(\delta \mathrm{I}_{i}, \mathrm{H}\right)_{\tau, \mathrm{T}}$ (figs. $40,41,42,43,44,4 \tilde{5}, \& 46$ (Pl. V.) in full lines) obtained from $\left(\delta \mathrm{I}_{i}, \tau\right)_{\mathrm{H}, \mathrm{T}}$ rise and fall steeply in a very low field, cut the axis of $\mathrm{H}$, become negative, and after passing through rather conspicuous negative maxima, slope away gradually toward the axis, with the increasing field. $\delta I$ is numerically greater for a greater twist. With 50.72 per cent. $\mathrm{Ni}$, however, $\delta \mathrm{I}$ for a small twist is always positive, tending to zero as the field increases. 
Curves $\left(\delta \mathrm{I}_{c}, \mathrm{H}\right)_{r, \mathrm{r}}$ for cyclic effect show $\mathrm{n}$ in the same figures are similar to the above curves, and become coincident with them above a moderate field. The increase of magnetization with small twists becomes less as the tension increases; and for $28 \cdot 74$ per cent. $\mathrm{Ni}$ and 70.32 per cent. $\mathrm{Ni}$, it almost vanishes at a high tension.

$28 \cdot 74$ per cent. Nickel-Steel. $\mathrm{T}=959 \mathrm{gr} . / \mathrm{mm} .^{2} ; t=14^{\circ} \cdot 2 \mathrm{C}$.

\begin{tabular}{|c|c|c|c|c|c|c|c|c|c|c|c|}
\hline \multicolumn{4}{|c|}{$\mathbf{H}^{\prime}=0 \cdot 10}$. & \multicolumn{4}{|c|}{$\mathrm{H}^{\prime}=0.21}$. & \multicolumn{4}{|c|}{$\mathrm{H}^{\prime}=0.48$} \\
\hline$\tau$. & $\delta \mathbf{I}_{i}$ & $\tau$ & $\delta \mathbf{I}_{c}$ & $\tau$. & $\delta \mathrm{I}_{i}$ & $\tau$ & $\delta \mathrm{I}_{c^{\prime}}$ & $\tau$. & $\delta \mathrm{I}_{i}$ & $\tau$ & $\delta \mathrm{I}_{c}$ \\
\hline $14 \cdot 5^{\prime}$ & $5 \cdot 4$ & $\ldots$ & $\ldots$ & $13 \cdot 2^{\prime}$ & $8 \cdot 6$ & $13 \cdot 4^{\prime}$ & $2 \cdot 1$ & $14 \cdot 8^{\prime}$ & $15 \cdot 8$ & $17 \cdot 6^{\prime}$ & $+1 \cdot 5$ \\
\hline $3+5$ & $12 \cdot 7$ & $36 \cdot 9^{\prime}$ & 049 & $38: 8$ & $23 \cdot 3$ & $35 \cdot 6$ & 27 & $40 \cdot 2$ & $28 \cdot 6$ & $41 \cdot 1$ & $-0 \cdot 1$ \\
\hline $68: 3$ & 18.9 & $68 \cdot 3$ & $1 \cdot 6$ & $68 \cdot 2$ & $36 \cdot 2$ & $68 \cdot 3$ & $2 \cdot 0$ & $68 \cdot 3$ & 31.0 & $68 \cdot 3$ & $-2 \cdot 7$ \\
\hline \multicolumn{4}{|c|}{$\mathrm{H}^{\prime}=1 \cdot 07$} & \multicolumn{4}{|c|}{$H^{\prime}=1 \cdot 86$} & \multicolumn{4}{|c|}{$\mathrm{H}^{\prime}=370$} \\
\hline T. & $\delta \mathrm{I}_{i}$ & $r$. & $\hat{\partial} \mathrm{I}_{c}$ & $T$. & $\delta \mathrm{I}_{i .}$ & $\tau$ & $\delta \mathrm{I}_{c}$ & $\tau$. & $\delta \mathrm{I}_{i^{*}}$ & $\tau$. & $\delta \mathbf{I}_{c}$ \\
\hline $13 \cdot 7^{\prime}$ & $+4 \cdot 8$ & $15 \cdot 8^{\prime}$ & $-2 \cdot 1$ & $17 \cdot 8^{\prime}$ & $-2 \cdot 4$ & $14 \cdot 5^{\prime}$ & $-3 \cdot 9$ & $15 \cdot 9^{\prime}$ & -53 & $15 \cdot 4^{\prime}$ & -54 \\
\hline $37 \cdot 4$ & $+2 \cdot 3$ & $35 \cdot 4$ & $-7 \cdot 2$ & $43 \cdot 6$ & $-12-2$ & $38 \cdot 2$ & $-13 \cdot 9$ & $56 \cdot 4$ & $-15 \cdot 9$ & $41 \cdot 8$ & $-19 \cdot 8$ \\
\hline $68 \cdot 3$ & $-4 \cdot 2$ & $68 \cdot 3$ & $-22 \cdot 0$ & $68 \cdot 3$ & $-20 \cdot 9$ & $68 \cdot 3$ & $-24 \cdot 6$ & $68 \cdot 3$ & $-30 \cdot 7$ & $68 \cdot 2$ & $-31 \cdot 7$ \\
\hline \multicolumn{4}{|c|}{$\mathrm{H}^{\prime}=6.70}$. & \multicolumn{4}{|c|}{$\mathrm{H}^{\prime}=14 \cdot 51}$. & \multicolumn{4}{|c|}{$\mathrm{H}^{\prime}=30.56$} \\
\hline$r$. & $\delta \mathrm{I}_{i}$ & $\tau$. & $\delta \mathrm{I}_{c^{\prime}}$ & $\tau$. & $\delta \mathbf{I}_{i}$ & $\tau$. & $\delta \mathrm{I}_{e}$ & $\tau$. & $\delta \mathrm{I}_{i}$ & $\tau$. & $\delta \mathrm{I}_{c}$ \\
\hline $14 \cdot 8^{\prime}$ & $-8 \cdot 9$ & $14 \cdot 7^{\prime}$ & $-4 \cdot 7$ & $13 \cdot \bar{s}^{\prime}$ & $-1 \cdot 3$ & $124^{\prime}$ & $-1 \cdot 1$ & $15 \cdot \vartheta^{\prime}$ & -0.4 & $18 \cdot 3^{\prime}$ & -0.9 \\
\hline $43 \cdot 4$ & $-18 \cdot 5$ & 367 & $-15 \cdot 7$ & $38 \cdot 7$ & $-7 \cdot 2$ & 387 & $-7 \cdot 6$ & $39 \cdot 4$ & -23 & $42 \cdot 1$ & -31 \\
\hline $682-$ & $-29 \cdot 9$ & 683 & $-30 \div 5$ & $68 \cdot 3$ & -158 & $68 \cdot 2$ & $-16 \cdot 6$ & $68 \cdot 2$ & -5.9 & $68 \cdot 2$ & $-6 \cdot 1$ \\
\hline \multicolumn{4}{|c|}{$\mathrm{H}^{\prime}=61 \cdot 0}$. & \multicolumn{4}{|c|}{$\mathrm{H}^{\prime}=168 \cdot 5$} & \multicolumn{4}{|c|}{$\mathrm{H}^{\prime}=35 \overline{5} \cdot 7$. } \\
\hline$\tau$. & $\delta \mathrm{I}_{i \bullet}$ & $\tau$. & $\delta 1_{c}$ & $T$. & $\delta \mathrm{I}_{i}$ & $\tau$. & $\delta \mathrm{I}_{c}$ & $\tau$ & $\delta \mathrm{I}_{i}$ & $\tau$. & $\partial \mathrm{I}_{c^{*}}$ \\
\hline $32 \cdot 9^{\prime}$ & -0.6 & $30 \cdot 4^{\prime}$ & $-0 \cdot 4$ & $37 \cdot 9^{\prime}$ & 0.0 & $\ldots$ & $\ldots$ & $36 \cdot 1^{\prime}$ & -0.1 & $\ldots$ & ... \\
\hline $63 \cdot 2$ & $-2 \cdot 1$ & $68 \cdot 2$ & $-2 \cdot 0$ & $68 \cdot 2$ & $-0 \cdot 1$ & $68 \cdot 2^{\prime}$ & $-0 \cdot 1$ & $68 \cdot 2$ & $-0 \cdot 1$ & $\ldots$ & $\ldots$ \\
\hline
\end{tabular}




$$
\mathrm{T}=4211 \mathrm{gr} . / \mathrm{mm} .{ }^{2} ; t=14^{\circ} \cdot 2 \mathrm{C} \text {. }
$$

\begin{tabular}{|c|c|c|c|c|c|c|c|}
\hline \multicolumn{2}{|c|}{$H^{\prime}=0 \cdot 13$} & \multicolumn{3}{|c|}{$\mathrm{H}^{\prime}=0 \cdot 24}$. & \multicolumn{3}{|c|}{$\mathrm{H}^{\prime}=0.48$} \\
\hline$\tau . \quad \delta \mathrm{I}_{i}$. & r. $\quad \delta \mathrm{I}_{c}$ & $\tau$. & $\delta I_{i}$ & $\tau . \quad \delta \mathrm{I}_{\sigma}$. & $\tau$. & $\delta \mathrm{I}_{i}$ & T. $\delta \mathrm{I}_{c}$. \\
\hline $14 \cdot 4^{\prime} \quad 8 \cdot 6$ & $17 \cdot 1^{\prime} \quad 0 \cdot 0$ & $14 \cdot 3^{\prime}$ & $16 \cdot 3$ & $15 \cdot 4^{\prime}-1 \cdot 3$ & $14 \cdot 9^{\prime}$ & $19 \cdot 7$ & $10^{\circ} \cdot 1^{\prime} \quad-4 \cdot 4$ \\
\hline $41 \cdot 2 \quad 29 \cdot 7$ & $39 \cdot 6$ & $40 \cdot 6$ & $46 \cdot 3$ & $40 \cdot 1 \quad-6 \cdot 9$ & $39 \cdot 2$ & $2 \pi \cdot 4$ & $39 \cdot 6-16 \cdot 7$ \\
\hline $68 \cdot 8 \quad 41 \cdot 7$ & $69 \cdot 0$ & $69 \cdot 0$ & $61 \cdot 0$ & $69 \cdot 0-12 \cdot 2$ & $69 \cdot 0$ & $22 \cdot 6$ & $69 \cdot 0-29 \cdot 9$ \\
\hline \multicolumn{2}{|c|}{$\mathrm{H}^{\prime}=1 \cdot 09$} & \multicolumn{3}{|c|}{$\mathbf{H}^{\prime}=1 \cdot 90$} & \multicolumn{3}{|c|}{$H^{\prime}=3 \cdot 60}$. \\
\hline$\tau . \quad \delta \mathrm{I}_{i}$ & ๘. $\delta \mathrm{I}_{c}$. & $\tau$. & $\delta \mathrm{I}_{i}$ & $\tau . \quad \delta \mathrm{I}_{c^{*}}$ & $\tau$ & $\delta \mathrm{I}_{i}$ & $\pi . \quad \delta \mathrm{I}_{c^{*}}$ \\
\hline $16 \cdot 1^{\prime}-1 \cdot 6$ & $15 \cdot 6^{\prime}-5 \cdot 1$ & $13 \cdot 6^{\prime}$ & $-2 \cdot 6$ & $18 \cdot 9^{\prime}-6 \cdot 1$ & $14 \cdot 9^{\prime}$ & -28 & $16 \cdot 5^{\prime}-4 \cdot 0$ \\
\hline $41 \cdot 6-14 \cdot 8$ & $38 \cdot 6-18 \cdot 7$ & $43 \cdot 4$ & $-15 \cdot 2$ & $42 \cdot 3-19 \cdot 2$ & $42 \cdot 6$ & $-15 \cdot 3$ & $40 \cdot 5-15 \cdot 2$ \\
\hline $69 \cdot 0-28 \cdot 9$ & $69 \cdot 0-34 \cdot 6$ & $69 \cdot 0$ & $-31 \cdot 2$ & $69 \cdot 0-33 \cdot 2$ & $69 \cdot 0$ & $-27 \cdot 7$ & $69 \cdot 0-29 \cdot 1$ \\
\hline \multicolumn{2}{|c|}{$\mathrm{H}^{\prime}=6 \cdot 78}$. & \multicolumn{3}{|c|}{$\mathrm{H}^{\prime}=14 \%$} & \multicolumn{3}{|c|}{$\mathrm{H}^{\prime}=3 \check{5} .58$} \\
\hline$\tau . \quad \delta \mathrm{I}_{i}$. & $\partial \mathrm{I}_{\boldsymbol{c}}$ & $\tau$. & $\delta \mathrm{I}_{i}$ & $\delta \mathrm{I}_{c}$. & $\tau$. & $\delta \mathrm{I}_{i}$ & $\delta \mathrm{I}_{c}$ \\
\hline $14 \cdot 2^{\prime}-2 \cdot 3$ & $17 \cdot 1^{\prime}-27$ & $14 \cdot 9^{\prime}$ & $-0 \cdot 9$ & $16 \cdot 6^{\prime}-1 \cdot 2$ & $18 \cdot 6^{\prime}$ & $-0 \cdot 4$ & $20 \cdot 6^{\prime}-0 \cdot 3$ \\
\hline $41 \cdot 7-11 \cdot 0$ & $40 \cdot 5-10 \cdot 4$ & $38 \cdot 8$ & $-4: 5$ & $\begin{array}{ll}38 \cdot 8 & -4 \cdot 8\end{array}$ & $46 \cdot 0$ & $-1 \cdot 7$ & $43 \cdot 5 \quad-1 \cdot 7$ \\
\hline $69 \cdot 0-21 \cdot 8$ & $69 \cdot 0-21.5$ & $69 \cdot 0$ & $-11 \cdot 0$ & $69 \cdot 0-11 \cdot 2$ & $69 \cdot 0$ & $-3 \cdot 3$ & $69 \cdot 0 \quad-3 \cdot 5$ \\
\hline \multicolumn{2}{|c|}{$\mathrm{H}^{\prime}=66.7}$. & \multicolumn{3}{|c|}{$\mathbf{H}^{\prime}=175^{\cdot} 9$} & \multicolumn{3}{|c|}{$\mathrm{H}^{\prime}=354 \cdot 6$} \\
\hline т. $\delta \mathrm{I}_{i}$ & $\delta \mathrm{I}_{\sigma}$ & $\tau$ & $\delta \mathrm{I}_{i}$ & $\delta \mathrm{I}_{c}$ & $\tau$ & $\delta \mathrm{I}_{i}$ & $\delta \mathrm{I}_{c}$ \\
\hline $19 \cdot 7^{\prime}-0 \cdot 1$ & $26 \cdot 3^{\prime} \quad 0 \cdot 0$ & $15 \cdot 9^{\prime}$ & $0 \cdot 0$ & $\ldots$ & ... & $\ldots$ & $\ldots$ \\
\hline $69 \cdot 0-1 \cdot 2$ & $69 \cdot 0-1 \cdot 1$ & $69 \cdot 0$ & 0.0 & $69 \cdot 0^{\prime}-0 \cdot 2$ & $69 \cdot 0^{\prime}$ & $0 \cdot 0$ & $69 \cdot 0^{\prime}-0 \cdot 2$ \\
\hline
\end{tabular}


$50 \cdot 72$ per cent. Nickel-Steel.

$\mathrm{T}=1151 \mathrm{gr} . / \mathrm{mm}^{2} ; t=14^{\circ} \cdot 3 \mathrm{C}$.

\begin{tabular}{|c|c|c|c|c|c|c|c|c|c|c|c|}
\hline \multicolumn{4}{|c|}{$\mathbf{H}^{\prime}=0 \cdot 28}$. & \multicolumn{4}{|c|}{$H^{\prime}=0.68}$. & \multicolumn{4}{|c|}{$\mathrm{H}^{\prime}=1 \cdot 38}$. \\
\hline$\tau$. & $\delta I_{i}$ & $\tau$. & $\delta \mathbf{I}_{c}$ & $\tau$. & $\delta \mathrm{I}_{i}$ & $\tau$ & $\delta \mathrm{I}_{c}$ & $\tau$. & $\delta \mathrm{I}_{i}$ & $\tau$. & $\delta \mathrm{I}_{G}$. \\
\hline $14 \cdot l^{\prime}$ & $22 \cdot 4$ & $17 \cdot 2^{\prime}$ & $8 \cdot 7$ & $12 \cdot 8^{\prime}$ & $51 \cdot 3$ & $10-4^{\prime}$ & $6 \cdot 2$ & $13 \cdot 0^{\prime}$ & $55 \cdot 9$ & $10 \mathbf{s}^{\prime}$ & $9 \cdot 3$ \\
\hline $29 \cdot 4$ & 57.7 & $35 \cdot 2$ & $19 \cdot 8$ & $27 \cdot 6$ & 118.8 & 307 & 169 & $26 \cdot 9$ & $117 \cdot 9$ & $32 \cdot 5$ & $23 \cdot 1$ \\
\hline $43 \cdot 2$ & $100 \cdot 2$ & 53.3 & $30 \cdot 3$ & $54 \cdot 3$ & $254 \cdot 1$ & $48 \cdot 9$ & $21 \cdot 8$ & $47^{* 4}$ & $199 \cdot 0$ & $54 \cdot 6$ & $11 \cdot 3$ \\
\hline $67 \cdot 9$ & $171 \cdot 7$ & $68 \cdot 2$ & $34 \cdot 7$ & 68.0 & 3056 & 68.1 & 16.2 & 67. & 2250 & $67 \cdot 9$ & $-3 \cdot 9$ \\
\hline \multicolumn{4}{|c|}{$\mathrm{H}^{\prime}=1 \cdot 83}$. & \multicolumn{4}{|c|}{$\mathrm{H}^{\prime}=3 \cdot 43}$. & \multicolumn{4}{|c|}{$\mathrm{H}^{\prime}=5 \cdot 46}$. \\
\hline$\tau$ & $\delta \mathrm{I}_{i}$ & $\tau$ & $\delta \mathrm{I}_{c}$ & $\tau$. & $\delta \mathbf{I}_{i}$ & $\pi$ & $\delta \mathrm{I}_{c}$ & $r$. & $\delta \mathrm{I}_{i}$ & $\tau$. & $\delta \mathrm{I}_{c}$ \\
\hline $10 \cdot 7^{\prime}$ & $42 \cdot 2$ & $14 \cdot 2^{\prime}$ & $15 \cdot 2$ & $14 \cdot 8^{\prime}$ & $35 \cdot 6$ & $12 \cdot 4^{\prime}$ & $16 \cdot 6$ & $10 \cdot 9^{\prime}$ & $19 \cdot 8$ & $13 \cdot 9^{\prime}$ & $17 \cdot 4$ \\
\hline $26 \cdot 9$ & 1068 & $34 \cdot 6$ & $27 \cdot 3$ & $29 \cdot 3$ & 567 & $27 \cdot 8$ & $28 \cdot 7$ & $26 \cdot 1$ & $38 \cdot 1$ & $31 \cdot 4$ & $26 \cdot 6$ \\
\hline 46.0 & $149 \cdot 6$ & $51 \cdot 4$ & $18 \cdot 0$ & $45 \cdot 7$ & $60 \cdot 8$ & $49 \cdot 0$ & $19 \cdot 7$ & $51 \cdot 0$ & $33 \cdot 6$ & 496 & $15 \%$ \\
\hline $67 \cdot 7$ & 1509 & $67 \cdot 9$ & $-4 \cdot 7$ & $67 \cdot 9$ & $37 \cdot 8$ & 67.7 & $-9 \cdot 0$ & $67 \cdot 7$ & $12 \cdot 5$ & $67 \cdot 7$ & $-9 \cdot 6$ \\
\hline \multicolumn{4}{|c|}{$H^{\prime}=12 \cdot 62}$. & \multicolumn{4}{|c|}{$H^{\prime}=24.57$} & \multicolumn{4}{|c|}{$\mathrm{H}^{\prime}=49 \cdot 61}$. \\
\hline$\pi$. & $\delta \mathrm{T}_{i}$ & $\tau$ & $\delta \mathrm{I}_{c}$ & $\tau$. & $\delta \mathrm{I}_{i}$ & $\pi$ & $\delta \mathrm{I}_{c^{*}}$ & $\pi$. & $\delta \mathrm{I}_{i}$ & $\tau$. & $\delta \mathrm{I}_{c}$ \\
\hline $12 \cdot 0^{\prime}$ & $13 \cdot 2$ & $9 \cdot 8^{\prime}$ & $8 \cdot 6$ & $12 \cdot 0^{\prime}$ & $8 \cdot 1$ & $16 \cdot 6^{\prime}$ & $10 \cdot 1$ & $15 \cdot 2^{\prime}$ & 45 & $17 \cdot 7^{\prime}$ & $4 \cdot 8$ \\
\hline & $22 \cdot 8$ & & 18.9 & $40 \cdot 6$ & $12 \cdot 0$ & 39.7 & $12 \cdot 0$ & $40 \cdot 4$ & $5 \cdot 7$ & $39 \cdot 0$ & 5.4 \\
\hline $52 \cdot 4$ & $13 \cdot 8$ & $54 \cdot 3$ & 6.8 & $\ldots$ & $\ldots$ & $\ldots$ & $\ldots$ & $\ldots$ & $\ldots$ & $\cdots$ & $\ldots$ \\
\hline $67 \cdot 7$ & -27 & $67 \cdot 7$ & $-8 \cdot 3$ & $67 \cdot 7$ & $-3 \cdot 6$ & $67 \cdot 7$ & $-4 \cdot 7$ & $67 \cdot 7$ & $-1 \cdot 8$ & $67 \cdot 7$ & $-2 \cdot 7$ \\
\hline \multicolumn{4}{|c|}{$\mathbf{H}^{\prime}=108 \cdot 8}$. & \multicolumn{4}{|c|}{$\mathrm{H}^{\prime}=211 \cdot 8$} & \multicolumn{4}{|c|}{$\mathrm{H}^{\prime}=377 \cdot 4}$. \\
\hline$\tau$. & $i \mathrm{I}_{i}$ & & $\delta \mathrm{I}_{c}$ & $\tau$. & $\delta \mathrm{I}_{i}$ & $r$. & $\delta \mathrm{I}_{c}$ & $\tau$. & $\delta \mathrm{I}_{\boldsymbol{i}}$ & $\tau$. & $\delta \mathrm{T}_{\sigma}$. \\
\hline $167^{\prime}$ & 1.7 & $15 \cdot \sigma^{\prime}$ & 1.5 & $157^{\prime}$ & $0 \cdot 7$ & $15 \cdot 8^{\prime}$ & $0 \cdot 6$ & $10 \cdot 9^{\prime}$ & $-0 \div$ & $\ldots$ & $\cdots$ \\
\hline $43 \cdot 1$ & 1.7 & $43 \cdot 6$ & 1.5 & $45 \cdot 6$ & 0.7 & 436 & $0 \cdot 6$ & $\ldots$ & $\ldots$ & $\ldots$ & $\cdots$ \\
\hline 67.7 & $-1 \cdot 1$ & $67 \cdot 7$ & -1.5 & $67 \cdot 7$ & $-0 \cdot 2$ & $67 \cdot 7$ & $-0 \cdot 3$ & $67 \cdot 7$ & $-0 \cdot 2$ & $\ldots$ & $\cdots$ \\
\hline
\end{tabular}


$\mathrm{T}=3409 \mathrm{gr} . / \mathrm{mm} .{ }^{2} ; t=14^{\circ} \cdot 3 \mathrm{C}$.

\begin{tabular}{|c|c|c|c|c|c|c|c|c|c|c|c|}
\hline \multicolumn{4}{|c|}{$\mathrm{H}^{\prime}=0.28$} & \multicolumn{4}{|c|}{$\mathrm{H}^{\prime}=0 \cdot 69}$. & \multicolumn{4}{|c|}{$H^{\prime}=1 \cdot 28}$. \\
\hline$\tau$. & $\delta \mathbf{I}_{i}$ & $T$. & $\delta \mathrm{I}_{C}$ & $\tau$. & $\delta \mathrm{I}_{i}$ & $\tau$. & $\delta \mathrm{I}_{\boldsymbol{c}}$ & $\tau$. & $\delta \mathrm{I}_{i}$ & $\tau$. & $\hat{\delta} \mathrm{I}_{c^{\prime}}$ \\
\hline $12 \cdot 4^{\prime}$ & 135 & $11^{\prime} 2^{\prime}$ & $2 \cdot 1$ & $12 \cdot 6^{\prime}$ & $38 \cdot 2$ & $11 \cdot 1^{\prime}$ & 4.5 & $121^{\circ}$ & $35 \cdot 0$ & $13 \cdot 5^{\prime}$ & $9 \cdot 3$ \\
\hline 297 & $42 \cdot 4$ & $30 \cdot 1$ & $4 \cdot 1$ & $28 \cdot 9$ & $88 \cdot 3$ & $27 \cdot 7$ & $9 \cdot 0$ & $27 \cdot 7$ & $83 \cdot 6$ & $30 \cdot 7$ & 155 \\
\hline $52 \cdot 0$ & $91 \cdot 7$ & $46 \cdot 2$ & 56 & 46.0 & $131 \cdot 6$ & 46.5 & $9 \cdot 0$ & $43 \cdot 3$ & $122 \cdot 8$ & $49 \cdot 6$ & $11 \cdot 4$ \\
\hline $67 \cdot 9$ & $121 \cdot 5$ & $67 \cdot 9$ & 56 & 67.9 & $182 \cdot 9$ & $67 \cdot 9$ & $3 \cdot 0$ & $67 \cdot 9$ & 1532 & $67 \cdot 9$ & -26 \\
\hline \multicolumn{4}{|c|}{$H^{\prime}=1 \cdot 82}$. & \multicolumn{4}{|c|}{$\mathrm{H}^{\prime}=4 \cdot 16$} & \multicolumn{4}{|c|}{$\mathrm{H}^{\prime}=10.54}$. \\
\hline$\tau$. & $\delta \mathrm{I}_{i}$ & $\tau$ & $\delta \mathrm{I}_{c^{*}}$ & $\tau$. & $\delta \mathrm{I}_{i}$ & $\tau$ & $\delta \mathrm{I}_{c}$ & $\tau$ & $\delta \mathrm{I}_{i}$ & $\tau$ & $\delta \mathbf{I}_{c}$ \\
\hline $11 \cdot 9^{\prime}$ & $35 \cdot 6$ & $10 \cdot 7^{\prime}$ & $9 \cdot 5$ & $11 \cdot 0^{\prime}$ & $18 \cdot 0$ & $11 \cdot 2^{\prime}$ & $11 \cdot 7$ & $112^{\prime}$ & $10 \cdot 1$ & $11 \cdot 8^{\prime}$ & 90 \\
\hline $31 \cdot 2$ & $81 \cdot 3$ & 26.9 & 186 & $27 \cdot 8$ & 35.0 & $28 \cdot 0$ & $22 \cdot 0$ & $29 \cdot 1$ & $18 \cdot 1$ & $38 \cdot 7$ & $16 \cdot 3$ \\
\hline $53 \cdot 0$ & $108 \cdot 0$ & 46.5 & $15 \cdot 2$ & 465 & $36 \cdot 1$ & $47 \cdot 9$ & 155 & $47 \cdot 6$ & $13 \cdot 8$ & $50 \cdot 5$ & $8 \cdot 9$ \\
\hline $67 \cdot 9$ & $108 \cdot 2$ & $67 \cdot 9$ & -47 & $67 \cdot 9$ & $18 \cdot 0$ & $67 \cdot 9$ & $-5 \cdot 6$ & 67.7 & $-2 \cdot 7$ & $67 \cdot 7$ & $-6 \cdot 2$ \\
\hline \multicolumn{4}{|c|}{$\mathrm{H}^{\prime}=24 \cdot 62}$. & \multicolumn{4}{|c|}{$\mathrm{H}^{\prime}=49 \cdot 35}$. & \multicolumn{4}{|c|}{$H^{\prime}=109 \cdot 0}$. \\
\hline$\tau$. & $\delta \mathrm{I}_{i}$ & $\tau$ & $\delta \mathrm{I}_{c^{\circ}}$ & $\tau$. & $\delta \mathrm{I}_{i}$ & $\tau$ & $\delta \mathrm{I}_{\boldsymbol{c}}$ & $\tau$. & $\delta \mathrm{I}_{i}$ & $\tau$. & $\delta \mathbf{I}_{G}$ \\
\hline $11 \cdot 3^{\prime}$ & $5 \cdot 7$ & $10 \cdot 9^{\prime}$ & $4 \cdot 8$ & $11 \cdot 8^{\prime}$ & $3 \cdot 0$ & $107^{\prime}$ & $2 \cdot 7$ & $15 \cdot 2^{\prime}$ & 1.5 & $12 \cdot 0^{\prime}$ & \\
\hline 27.7 & $9 \cdot 8$ & $28 \cdot 6$ & $8 \cdot 9$ & $28 \cdot 0$ & $4 \cdot 5$ & $25 \cdot 4$ & $4 \cdot 8$ & 41.9 & 1.5 & $44 \cdot 2$ & $1 \cdot 4$ \\
\hline $47 \cdot 3$ & 6.9 & $48 \cdot 6$ & $5 \cdot 7$ & $51 \cdot 0$ & $2 \cdot 4$ & 46.7 & 35 & $\ldots$ & $\ldots$ & $\ldots$ & $\ldots$ \\
\hline $67 \cdot 7$ & $-3 \cdot 3$ & $67 \cdot 7$ & $-4 \cdot 2$ & $67 \cdot 7$ & $-2 \cdot 4$ & $67 \cdot 9$ & -23 & 67.7 & -0.6 & $67 \cdot 7$ & -0.5 \\
\hline & & \multicolumn{4}{|c|}{$H^{\prime}=212 \cdot 8}$. & \multicolumn{4}{|c|}{$\mathrm{H}^{\prime}=376 \cdot 8$} & & \\
\hline & & $\tau$ & $\delta \mathrm{I}_{i}$ & $\tau$. & $\delta \mathrm{I}_{c}$ & $\tau$. & $\delta \mathbf{I}_{i}$ & $r$. & $\delta \mathrm{I}_{0}$ & & \\
\hline & & $177^{\prime}$ & & $13 \cdot 2^{\prime}$ & 0.8 & $23 \cdot 0^{\prime}$ & 0.0 & $21 \cdot 9^{\prime}$ & 00 & & \\
\hline & & $35 \cdot 1$ & 03 & $37 \cdot 8$ & 0.8 & $\ldots$ & $\cdots$ & $\ldots$ & $\ldots$ & & \\
\hline & & $67 \cdot 7$ & $-0 \cdot 8$ & $67 \cdot 7$ & 03 & $67 \cdot 7$ & -0.3 & $67 \cdot 7$ & -0.2 & & \\
\hline
\end{tabular}


70.32 per cent. Nickel.

$\mathrm{T}=1123 \mathrm{gr} . / \mathrm{mm} .^{2} ; t=12 \% 7 \mathrm{C}$.

\begin{tabular}{|c|c|c|c|c|c|c|c|}
\hline \multicolumn{4}{|c|}{$\mathrm{H}^{\prime}=0.29$} & \multicolumn{2}{|c|}{$H^{\prime}=0 \cdot 69$} & \multicolumn{2}{|c|}{$\mathrm{H}^{\prime}=0.89}$. \\
\hline$\tau$ & $\delta \mathrm{I}_{i}$ & $\tau$ & $\delta \mathrm{I}_{\iota}$ & $\dot{\tau} . \quad \dot{i} \mathrm{I}_{i}$. & $\tau . \quad \dot{i} I_{c}$ & r. $\quad \dot{i} \mathrm{I}_{i}$. & T. $\delta \mathrm{I}_{c}$ \\
\hline $22 \cdot 6^{\prime}$ & $77 \cdot 0$ & $25 \cdot 3^{\prime}$ & $4 \cdot 4$ & $162^{\prime} \quad 89 \cdot 0$ & $23 \cdot 2^{\prime} \quad 14 \cdot 1$ & $16 \cdot 6^{\prime} \quad 128 \cdot 2$ & $17 \cdot 9^{\prime} \quad 7 \cdot 9$ \\
\hline$\ldots$ & $\ldots$ & $\ldots$ & $\ldots$ & $35.5 \quad 212.7$ & $\ldots$ & $37 \cdot 3 \quad 321 \cdot 0$ & $42 \cdot 0 \quad 32 \cdot 7$ \\
\hline $48 \cdot 5$ & $118 \cdot 1$ & $48 \cdot 9$ & 19 & $54: 5 \quad 2950$ & $49 \cdot 4 \quad 34 \cdot 0$ & $557 \quad 394.6$ & $\ldots$ \\
\hline $69 \cdot 3$ & 121.9 & $69 \cdot 3$ & $-1 \cdot 0$ & $693 \quad 341.0$ & $69 \cdot 3 \quad 42 \cdot 9$ & $69 \cdot 3 \quad 4: 0 \cdot 8$ & $69 \cdot 0 \quad 44 \cdot 5$ \\
\hline \multicolumn{4}{|c|}{$H^{\prime}=1 \cdot 70$} & \multicolumn{2}{|c|}{$H^{\prime}=3 \cdot 08$} & \multicolumn{2}{|c|}{$\mathrm{H}^{\circ}=4 \cdot 64}$. \\
\hline$\tau$ & $\delta \mathrm{I}_{i}$ & $\tau$ & $\delta \mathrm{I}_{c}$ & $\tau . \quad \delta \mathrm{I}_{i}$. & $\tau . \quad \delta \mathrm{I}_{c^{*}}$ & $\delta I_{i}$ & $\tau . \quad \delta \mathbf{I}_{c^{\prime}}$ \\
\hline $143^{\prime}$ & $49 \cdot 3$ & $256^{\prime}$ & $5 \cdot 1$ & $227^{\prime} \quad 199$ & $184^{\prime}-3 \cdot 2$ & $201^{\prime} \quad 40$ & $21 \cdot 0^{\prime}-9 \cdot 0$ \\
\hline $37 \cdot 1$ & 98.8 & $48 \cdot 8$ & $10 \cdot 6$ & $48 \cdot 7 \quad 22 \cdot 8$ & $44 \cdot 9-14 \cdot 8$ & $36 \cdot 1-8 \cdot 1$ & $47 \cdot 6-33 \cdot 6$ \\
\hline 55.9 & $110 \cdot 2$ & $\ldots$ & $\cdots$ & $\ldots$ & $\begin{array}{ll}\ldots & \ldots\end{array}$ & $\ldots \quad \ldots$ & $\begin{array}{ll}\ldots & \ldots\end{array}$ \\
\hline $69 \cdot 3$ & $115 \cdot 0$ & $69 \cdot 3$ & $13 \cdot 0$ & \begin{tabular}{|ll}
$69 \cdot 4$ & $20 \cdot 2$
\end{tabular} & $69+4-220$ & $69 \cdot 4-27 \cdot 6$ & $69 \cdot 3-44 \cdot 9$ \\
\hline \multicolumn{4}{|c|}{$H^{\prime}=8.96}$. & \multicolumn{2}{|c|}{$\mathrm{H}^{\prime}=23 \cdot 23$} & \multicolumn{2}{|c|}{$\mathrm{H}^{\prime}=47.04}$. \\
\hline$\tau$ & $\delta \mathrm{I}_{i}$ & $\tau$ & $\delta \mathrm{I}_{e^{\prime}}$ & $\tau . \quad \delta \mathrm{I}_{i}$ & $\bar{\tau} \quad \delta \mathrm{T}_{c}$ & $\delta I_{i}$ & T. $\delta \mathrm{I}_{c^{*}}$ \\
\hline $18 \cdot 5^{\prime}$ & -5.8 & $25 \cdot 1^{\prime}$ & $-17 \cdot 1$ & $25 \cdot 8^{\prime}-10 \cdot 2$ & $20^{\prime} 1^{\prime}-5 \cdot 2$ & $161^{\prime}-25$ & $21 \cdot 2^{\prime}-2.5$ \\
\hline $45 \cdot 8$ & $-44 \cdot 9$ & $48 \cdot 5$ & $-49 \cdot 4$ & $46 \cdot 5-35 \cdot 1$ & $48 \cdot 0-35 \cdot 8$ & $47 \cdot 5-17 \cdot 0$ & $47 \cdot 9-17 \cdot 8$ \\
\hline $69 \cdot 3-$ & $-67 \cdot 2$ & $69 \cdot 1$ & $-69 \cdot 4$ & $\left|\begin{array}{|cc}693 & -60 \cdot 2\end{array}\right|$ & $69 \cdot 3-60 \cdot 8$ & $69 \cdot 3-32 \cdot 6$ & $69 \cdot 3-332$ \\
\hline \multicolumn{4}{|c|}{$\mathrm{H}^{\prime}=92 \cdot 0}$. & \multicolumn{2}{|c|}{$\mathrm{H}^{\prime}=208 \cdot 7$} & \multicolumn{2}{|c|}{$H^{\prime}=367 \cdot 2}$. \\
\hline$\tau$ & $\delta \mathbf{I}_{i}$ & $\tau$ & $\delta \mathrm{I}_{c}$ & T. $\quad \delta \mathrm{I}_{i}$ & t. $\delta \mathrm{I}_{c}$. & $\dot{\delta} \mathrm{I}_{i}$ & $\delta \mathbf{I}_{c}$ \\
\hline $26 \cdot 2^{\prime}$ & -1.5 & $\ldots$ & $\cdots$ & $\begin{array}{ll}25.3^{\prime} & 0.0 \\
\end{array}$ & $\ldots$ & $\ldots$ & $\ldots$ \\
\hline $48 \cdot 7$ & $-6 \cdot 4$ & $\ldots$ & ... & $49 \cdot 5-1 \cdot 5$ & $\ldots$ & $\begin{array}{ll}\cdots & \ldots\end{array}$ & $\ldots$ \\
\hline $69 \cdot 3$ & $-13 \cdot 2$ & & $\cdots$ & $69 \cdot 3 \quad-3 \cdot 0$ & $\cdots$ & $69 \cdot 3^{\prime}-1 \cdot 3$ & $\cdots$ \\
\hline
\end{tabular}




$$
\mathrm{T}=3366 \mathrm{gr} . / \mathrm{mm} .^{2} ; t=12^{\circ} \cdot 7 \mathrm{C} \text {. }
$$

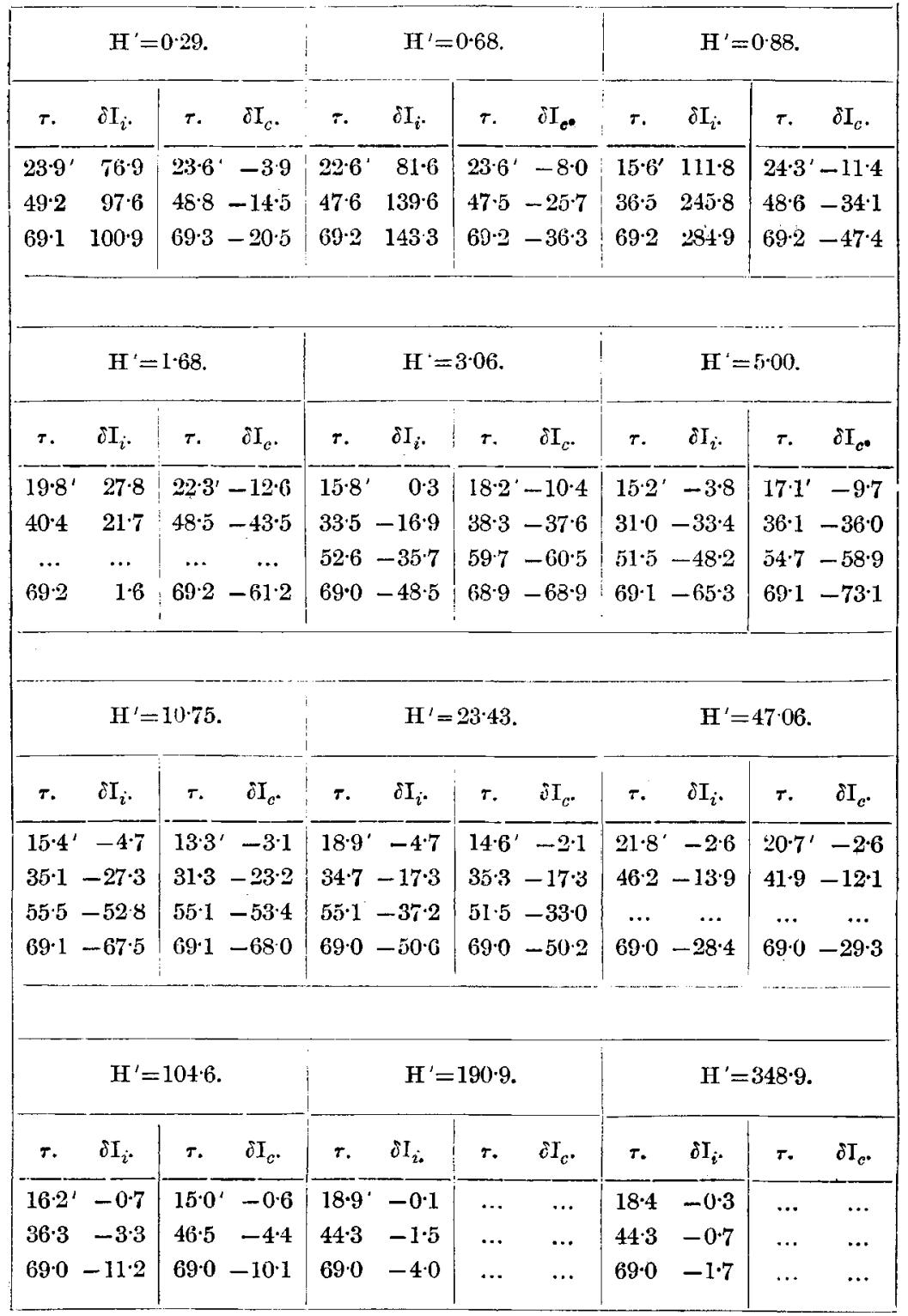


Effect of Stress on Magnetization.

The effect of giving a maximum twist at once does not materially differ from that of a graded twisting.

\section{(d) Magnetization under Constant Twist combined with Tensions: $(\mathrm{I}, \mathrm{H})_{\tau, \mathrm{r}}$.}

The effect of a constant twist on magnetization is comparatively great, especially in high fields. In $28 \cdot 74$ per cent. $\mathrm{Ni}$, the magnetization is slightly increased by a small twist, but above a moderate twist it decreases. In $50 \cdot 72$ per cent. $\mathrm{Ni}$, the increase of magnetization by a small twist is not appreciable, but the magnetization always decreases with greater twist. The magnetization of $70 \cdot 32$ per cent. $\mathrm{Ni}$ is also decreased by twisting, except in weak fields in which a slight increase is observed. In all cases, the change of magnetization decreases with increasing tension.

In 28.74 per cent. $\mathrm{Ni}$, curves $(\delta \mathrm{I}, \mathrm{H})_{\tau, \mathrm{r}}(\mathrm{fig} .40$ in dotted lines) deduced from $(\mathrm{I}, \mathrm{H})_{\tau, \mathrm{r}}$ show a somewhat different aspect from those deduced from $(\delta I, \tau)_{\text {T.H. }}$, especially for a small twist. For a small value of twist, $\delta I$ is always positive and has a faint maximum; for a greater twist it is first negative and afterward positive; and for a still greater twist it is always negative, and, except in weak fields, it takes a course parallel to the corresponding curve obtained from the last experiment, but the former lies somewhat below the latter.

In 50.72 per cent. $\mathrm{Ni}$, curves $(\delta \mathrm{I}, \mathrm{H})_{\tau, \mathrm{T}}$ (figs. 41 and 42 in dotted lines) deduced from $(\mathrm{I}, \mathrm{H})_{\boldsymbol{\tau}, \boldsymbol{r}}$ have a quite different aspect, $i_{.} e . \delta \mathrm{I}$ is always negative. It rapidly decreases in weak fields, and after passing through a negative maximum, slopes away very slowly towards the axis of $\mathrm{H}$ with increasing field. Tension reduces the decrease of magnetization.

In $70 \cdot 32$ per cent. $\mathrm{Ni}$, curves $(\delta \mathrm{I}, \mathrm{H})_{\tau, \mathrm{r}}$ (figs. $43,44,45$, and 46 dotted lines) deduced from $(\mathrm{I}, \mathrm{H})_{\tau, \mathrm{r}}$ take a course similar to those obtained from the last experiment, but the difference is that in the former the positive maxima in weak fields are considerably smaller, the points at which $\delta I$ changes its sign lie in a lower part of the field, and $\delta I$ tends more slowly to zero than in the latter. The effect of tension is to push the points of intersection with the axis of $H$ towards the origin.

For these alloys curves $(\delta \mathrm{I}, \mathrm{H})_{\tau, \mathbf{T}}$ as deduced from $(\mathrm{I}, \mathrm{H})_{\tau, \mathbf{T}}$ rather resemble the curves $\left(\delta \mathrm{I}_{c}, \mathrm{H}\right)_{\tau, \mathrm{r}}$ obtained from the last experiment.

Phil. Mag. S. 6. Vol. 14. No. 79. July 1907. H 
28.74 per cent. Nickel-Steel. $\mathrm{T}=959 \mathrm{gr} . / \mathrm{mm}^{2} ; t=14^{\circ} \cdot 6 \mathrm{C}$.

\begin{tabular}{|c|c|c|c|c|c|c|c|}
\hline \multicolumn{2}{|c|}{$\tau=0}$. & \multicolumn{2}{|c|}{$\tau=11^{\prime} \cdot 4$} & \multicolumn{2}{|c|}{$\tau=34^{\prime} \cdot 8$} & \multicolumn{2}{|c|}{$\tau=68^{\prime} \cdot 0$} \\
\hline H. & I. & H. & I. & H. & I. & H. & I. \\
\hline 0.29 & $35 \cdot 6$ & 0.28 & 34.9 & $0 \cdot 29$ & $28 \cdot 1$ & 0.30 & $23 \cdot 6$ \\
\hline 0.42 & $82 \cdot 8$ & $0 \cdot 42$ & $85 \cdot 5$ & 039 & $77 \cdot 8$ & $0 \cdot 44$ & $63 \cdot 0$ \\
\hline 0.58 & 121.8 & 0.52 & $112 \cdot 4$ & 0.57 & $113 \cdot 8$ & 0.59 & $96 \cdot 6$ \\
\hline 0.90 & 165.8 & 0.85 & 165.3 & 089 & 162.2 & 0.92 & $137 \cdot 3$ \\
\hline $1 \cdot 34$ & $196 \%$ & $1 \cdot 19$ & 192.9 & $1 \cdot 41$ & $197 \cdot 0$ & $1 \cdot 64$ & $179 \cdot 5$ \\
\hline $2 \cdot 37$ & $235 \cdot 3$ & $3 \cdot 15$ & 261.9 & $2 \cdot 89$ & $243 \cdot 3$ & $2 \cdot 85$ & $211 \cdot 9$ \\
\hline $4 \cdot 54$ & 271.8 & $5 \cdot 35$ & $288 \cdot 9$ & 4.82 & 2726 & $4 \cdot 68$ & $238 \cdot 8$ \\
\hline 6.91 & $290 \cdot 3$ & 6.90 & $299 \cdot 6$ & $6 \cdot 75$ & 286.5 & $6 \cdot 89$ & $258 \cdot 3$ \\
\hline 18.93 & $314: 5$ & 19.04 & $322 \cdot 4$ & $19 \cdot 04$ & 318.7 & $19 \cdot 09$ & 297.8 \\
\hline $24 \cdot 15$ & 318.1 & $24 \cdot 30$ & 326.0 & $24 \cdot 18$ & $323 \cdot 1$ & $24 \cdot 35$ & $303 \cdot 8$ \\
\hline $49 \cdot 20$ & 326.8 & 50.00 & 334.9 & $50 \cdot 0$ & $332 \cdot 2$ & $50 \cdot 1$ & $316^{-4}$ \\
\hline $129 \cdot 4$ & $337 \cdot 7$ & 128.7 & 346.0 & $127 \cdot 3$ & $343 \overline{5}$ & $126 \cdot 6$ & $329 \cdot 0$ \\
\hline $239 \cdot 8$ & 350.5 & $238 \cdot 8$ & $359 \cdot 6$ & 236.7 & $357 \cdot 2$ & $234 \cdot 2$ & $342 \cdot 6$ \\
\hline $348 \cdot 5$ & $357 \cdot 0$ & $366 \cdot 1$ & $367 \cdot 5$ & $362 \cdot 6$ & 3647 & $341 \cdot 5$ & $349 \cdot 4$ \\
\hline
\end{tabular}

50.72 per cent. Nickel-Steel.

$\mathrm{T}=1151 \mathrm{gr} . / \mathrm{mm} .{ }^{2} ; t=13^{\circ} \cdot 3 \mathrm{C}$.

\begin{tabular}{|c|c|c|c|c|c|c|c|}
\hline \multicolumn{2}{|c|}{$\tau=0}$. & \multicolumn{2}{|c|}{$\tau=11^{\prime} \cdot 7$} & \multicolumn{2}{|c|}{$\tau=34^{\prime} \cdot 7$} & \multicolumn{2}{|c|}{$\tau=67^{\prime} \cdot 3$} \\
\hline H. & I. & H. & I. & H. & I. & H. & I. \\
\hline $0 \cdot 18$ & $16 \cdot 6$ & $0 \cdot 18$ & $15 \cdot 8$ & $0 \cdot 16$ & $13 \cdot 8$ & $0 \cdot 18$ & $12 \cdot 3$ \\
\hline 0.50 & 87.8 & 0.46 & $92 \cdot 6$ & 0.50 & $89 \cdot 3$ & 0.49 & $72 \cdot 6$ \\
\hline 0.65 & $197 \cdot 2$ & 0.66 & 2207 & 0.64 & $217 \cdot 3$ & 0.65 & $182 \cdot 1$ \\
\hline $0 \cdot 79$ & $271 \cdot 8$ & 0.96 & 528 & 0.75 & 399.5 & 0.91 & 4806 \\
\hline $1 \cdot 14$ & 659 & 1.08 & 635 & $1 \cdot 10$ & 624 & $1 \cdot 18$ & 585 \\
\hline $1 \cdot 43$ & 787 & $1 \cdot 37$ & 755 & $1 \cdot 45$ & 739 & 2.02 & 804 \\
\hline $2 \cdot 73$ & 1016 & 241 & 981 & 205 & 882 & 3.34 & 929 \\
\hline 3.90 & 1085 & 4.95 & 1115 & 3.56 & 1013 & 4.60 & 994 \\
\hline 6.04 & 1155 & 8.68 & 1193 & 6.42 & 1112 & $7 \cdot 84$ & $108 t$ \\
\hline $12 \cdot 39$ & 1240 & 1543 & 1251 & 1083 & 1183 & $14-20$ & 1163 \\
\hline 2258 & 1288 & 2161 & 1279 & $22 \cdot 82$ & 1257 & $2 \cdot 45$ & 1215 \\
\hline 39.94 & 1319 & $40 \cdot 74$ & 1316 & $40: 30$ & 1297 & 39.97 & 1268 \\
\hline 100.9 & 1345 & $99 \cdot 9$ & 1346 & $101 \cdot 3$ & 1332 & $101 \cdot 3$ & 1317 \\
\hline 216.9 & 1354 & $214: 5$ & 1350 & $217 \cdot 2$ & 1344 & $217 \cdot 2$ & 1334 \\
\hline $351 \cdot 6$ & 1358 & 349.8 & 1355 & 3537 & 1348 & $351 \cdot 1$ & 1340 \\
\hline
\end{tabular}


$\mathrm{T}=3409 \mathrm{gr} . / \mathrm{mm}^{2} ; t=13^{\circ} \cdot 3 \mathrm{C}$.

\begin{tabular}{|c|c|c|c|c|c|}
\hline \multicolumn{2}{|c|}{$\tau=0}$. & \multicolumn{2}{|c|}{$\tau=11^{\prime} \cdot 7$} & \multicolumn{2}{|c|}{$\tau=68^{\prime} \cdot 2$} \\
\hline H. & I. & H. & I. & H. & I. \\
\hline 0.21 & $12 \cdot 6$ & $0 \cdot 19$ & $14 \cdot 4$ & $0 \cdot 20$ & $26 \cdot 4$ \\
\hline 0.49 & 128.9 & 0.50 & $115 \cdot 6$ & 0.50 & $127 \cdot 8$ \\
\hline 0.61 & 296.9 & 0.62 & $309 \cdot 6$ & 0.62 & $330 \cdot 7$ \\
\hline 0.90 & 676 & 0.79 & 578 & 1.02 & 671 \\
\hline $1 \cdot 13$ & 863 & 1.02 & 751 & $1 \cdot 37$ & 789 \\
\hline $2 \cdot 43$ & 1073 & 1.52 & 932 & $1 \cdot 64$ & 859 \\
\hline $3 \cdot 16$ & 1125 & $2 \cdot 44$ & 1070 & $3 \cdot 22$ & 1031 \\
\hline 4.47 & 1169 & 5.04 & 1178 & $4: 34$ & 1077 \\
\hline $7 \cdot 60$ & 1220 & 8.03 & 1219 & 8.08 & 1151 \\
\hline 1456 & 1256 & 13.09 & 1246 & 1351 & 1193 \\
\hline 22.08 & 1273 & $22 \cdot 43$ & 1270 & 2261 & 1229 \\
\hline 40.81 & 1291 & $40 \cdot 34$ & 1290 & 40.67 & 1264 \\
\hline $98 \cdot 1$ & 1308 & $97 \cdot 4$ & 1308 & $99 \cdot 2$ & 1300 \\
\hline 213.0 & 1317 & $209 \cdot 9$ & 1317 & 2137 & 1315 \\
\hline $343 \cdot 8$ & 1321 & $340 \cdot 7$ & 1321 & 345.9 & 1317 \\
\hline
\end{tabular}

70.32 per cent. Nickel-Steel.

$\mathrm{T}=1123 \mathrm{gr} . / \mathrm{mm}^{2} ; t=13^{\circ} \cdot 0 \mathrm{C}$.

\begin{tabular}{|c|c|c|c|c|c|c|c|}
\hline \multicolumn{2}{|c|}{$\tau=0}$. & \multicolumn{2}{|c|}{$\tau=13^{2} \pi}$. & \multicolumn{2}{|c|}{$\tau=35^{\prime} \cdot 5$} & \multicolumn{2}{|c|}{$\tau=68^{\prime} \cdot 3$} \\
\hline H. & I. & H. & I. & H. & I. & H. & I. \\
\hline $0 \cdot 25$ & $23 \cdot 6$ & $0 \cdot 20$ & $16 \cdot 2$ & 0.20 & $10 \cdot 0$ & 0.29 & $17 \cdot 1$ \\
\hline 0.74 & 161.9 & 0.65 & 114.4 & 0.65 & $144 \cdot 1$ & 0.63 & $108 \cdot 2$ \\
\hline 0.93 & $423 \cdot 4$ & 0.87 & 3027 & 0.91 & $376 \cdot 2$ & 0.95 & $310^{\circ} 2$ \\
\hline $1 \cdot 12$ & 575 & $1 \cdot 05$ & $55 \overline{5}$ & $1 \cdot 25$ & 672 & $1 \cdot 22$ & 689 \\
\hline 1.49 & 688 & $1 \cdot 27$ & 635 & $1 \cdot 50$ & 701 & $1 \cdot 47$ & 713 \\
\hline $2 \cdot 50$ & 809 & $2 \cdot 55$ & 790 & 261 & 774 & $2 \cdot 45$ & 764 \\
\hline 385 & 890 & $4 \cdot 35$ & 883 & 4.21 & 834 & 3.96 & 809 \\
\hline 8.07 & 988 & 6.87 & 939 & 7.09 & 888 & $7 \cdot 15$ & 858 \\
\hline 1264 & 1029 & $10 \cdot 46$ & 983 & 11.82 & 937 & 11.21 & 895 \\
\hline $17 \cdot 62$ & 1053 & $16 \cdot 66$ & 1020 & 16.97 & 970 & 16.99 & 930 \\
\hline 2271 & 1065 & 21.60 & 1037 & 21.86 & 990 & 21.03 & 951 \\
\hline 4673 & 1090 & $44 \cdot 44$ & 1065 & 44.90 & 1030 & $45 \cdot 18$ & 1005 \\
\hline 927 & 1098 & $87 \cdot 4$ & 1076 & $87 \cdot 1$ & 1047 & $87 \cdot 1$ & 1035 \\
\hline $181 \cdot 7$ & 1101 & 1746 & 1080 & $172 \cdot 9$ & 1053 & 172.5 & 1049 \\
\hline $368 \cdot 1$ & 1104 & 345.4 & 1081 & 342.9 & 1056 & $341 \cdot 2$ & 1055 \\
\hline
\end{tabular}




$$
\mathrm{T}=3366 \mathrm{gr} . / \mathrm{mm}^{2} ; t=13^{\circ} \cdot 0 \mathrm{C} .
$$

\begin{tabular}{|c|c|c|c|c|c|c|c|}
\hline \multicolumn{2}{|c|}{$r=0}$. & \multicolumn{2}{|c|}{$\tau=12^{\prime} \cdot 8$} & \multicolumn{2}{|c|}{$\tau=36^{\prime} \cdot 7}$. & \multicolumn{2}{|c|}{$\tau=69^{\prime} \cdot 0}$. \\
\hline H. & I. & H. & I. & H. & I. & H. & I. \\
\hline 0.21 & $9 \cdot 4$ & $0 \cdot 21$ & $9 \cdot 0$ & $0 \cdot 25$ & $31 \cdot 4$ & 0.22 & $6 \cdot 1$ \\
\hline 0.56 & $186 \cdot 2$ & 0.58 & $193 \cdot 6$ & 0.64 & 232.5 & 0.67 & $155 \cdot 9$ \\
\hline 0.74 & 4429 & 0.73 & 4366 & 071 & 528 & 0.72 & $245 \cdot 1$ \\
\hline 0.93 & 784 & 0.99 & 824 & 0.90 & 768 & 081 & $400 \cdot 8$ \\
\hline 1.33 & 863 & $1 \cdot 34$ & 866 & $1 \cdot 45$ & 839 & 0.94 & 767 \\
\hline $2: 40$ & 934 & $2 \cdot 18$ & 924 & $3 \cdot 25$ & 912 & 1.23 & 800 \\
\hline 364 & 973 & 370 & 972 & $4 \cdot 64$ & 936 & $2 \cdot 51$ & 863 \\
\hline 7.83 & 1016 & $7 \cdot 25$ & 1011 & $8 \cdot 37$ & 967 & $3 \cdot 75$ & 890 \\
\hline 11.86 & 1035 & $11 \cdot 44$ & 1033 & 1255 & 989 & $9 \cdot 74$ & 941 \\
\hline 1656 & 1048 & 1681 & 1047 & $17 \cdot 65$ & 1006 & $17 \cdot 63$ & 976 \\
\hline $21 \cdot 48$ & 1057 & $21 \cdot 78$ & 1056 & 2278 & 1018 & $22 \cdot 72$ & 991 \\
\hline 44.84 & 1074 & 44.83 & 1074 & $46 \cdot 43$ & 1044 & $46 \cdot 44$ & 1029 \\
\hline 86.7 & 1081 & 860 & 1082 & 89.9 & 1056 & 89.9 & 1051 \\
\hline 1708 & 1084 & $171 \cdot 5$ & 1084 & $17 \cdot 2$ & 1062 & $172 \cdot 2$ & 1062 \\
\hline $359 \cdot 8$ & 1086 & $361 \cdot 8$ & 1086 & $361 \cdot 7$ & 1065 & $364 \cdot 0$ & 1067 \\
\hline
\end{tabular}

Thus in the case of nickel-steels, the change of magnetization by tension does not differ much for the different orders of straining and magnetizing. So also in the change of elasticity, we found a fair agreement between the values for different orders, especially at high tensions. On the other hand, the change of magnetization by twist differs sometimes in a considerable degree for the different orders, while in the change of rigidity the agreement between the values for different orders is generally good, if the tension be large, especially in 28.74 and 70.32 per cent. of nickel. . In general, alloys, for which the hysteresis effect is small, have also in small difference in the changes of elastic constants by magnetization for the different orders of magnetizing and straining.

Thus far we have seen that generally the change of magnetization by stresses differs more or less with the different orders of applying the magnetic field and stress. In some cases, the difference is not only quantitative but also qualitative, as for the effect of twist in Swedish iron or in 50.72 per cent. $\mathrm{Ni}$, if the initial effect of twisting under constant field be compared with the results of magnetization under constant twist. On the other hand, there are examples of good coincidence, as in the case of the tension effect in nickel and 70.32 per cent. nickel-steel. Generally speaking, the tension effect shows a better agreement for the different orders of magnetizing and straining than for the torsion 
effect; and the discrepancy is remarkable in low magnetic fields, as may be expected from the consideration of the hysteresis effect prominent in that region.

In our preceding paper, we have remarked that the dependence of the change of elastic constants on the different orders of magnetizing and straining is probably due to the hysteresis effect accompanying magnetization. This explanation agrees well with the facts brought out by the present experiment.

\section{\$4. Reciprocal Relations.}

Among several important reciprocal relations obtained by J. J. Thomson, the two relations which have connexion with the present experiment are referred to below.

Let a cylindrical bar of soft iron, whose axis coincides with the axis of $x$, be magnetized along its axis. Let $e, f, g$, be the dilatations of the bar parallel to the axes of $x, y, z$ respectively; J. J. Thomson obtained the relation

$$
\begin{aligned}
n \frac{\partial e}{\partial \mathrm{I}^{2}}=\frac{1}{2}\left\{1-\mathrm{H}\left(\frac{\partial \kappa}{\partial \mathrm{I}}\right)_{e_{,}, g}\right\}\left\{\frac{m}{3 m-n} \frac{1}{\kappa \mathrm{I}}\left(\frac{\partial \mathrm{I}}{\partial e}\right)_{\mathrm{H} . f, g}\right. \\
\left.-\frac{m-n}{3 m-n} \frac{1}{\kappa \mathrm{I}}\left(\frac{\partial \mathrm{I}}{\partial f}\right)_{\mathrm{H}, e, g}\right\}, . .
\end{aligned}
$$

where $\mathrm{I}, \mathrm{H}, \boldsymbol{\kappa}$ have the usual meanings, $n$ represents the coefficient of rigidity, and $m$ is connected with the modulus of compression $k$ by the relation $k=m-n / 3$. In his original work the factor $\frac{1}{2}$ is dropped in the right-hand member of the above equation; the error is to be traced back to his equation (41).

Since $d I=\kappa d \mathrm{H}+\mathrm{H} d \kappa$, we have the relation, supposing the strain to be kept constant,

$$
1-\mathrm{H}\left(\frac{\partial \kappa}{\partial \mathrm{I}}\right)_{e, f, g}=\frac{\kappa}{\left(\frac{\partial \mathrm{I}}{\partial \mathrm{H}}\right)_{e, f, g}} .
$$

Hence equation (1) may be written

$\frac{\partial e}{\partial \mathrm{I}}\left(\frac{\partial \mathrm{I}}{\partial \mathrm{H}}\right)_{e, f, g}=\frac{m}{n(3 m-n)}\left(\frac{\partial \mathrm{I}}{\partial e}\right)_{\mathrm{H}, f, g}-\frac{m-n}{n(3 m-n)}\left(\frac{\partial \mathrm{I}}{\partial f}\right)_{\mathbf{H}, e, g} \cdot$

Again, if $\mathrm{T}$ is the tension per unit of area, we have 


$$
\begin{aligned}
\left(\frac{\partial \mathrm{I}}{\partial \mathrm{T}}\right)_{\mathrm{H}} & =\left(\frac{\partial \mathrm{I}}{\partial e}\right)_{\mathrm{H}, f, g}\left(\frac{\partial e}{\partial \mathrm{T}}\right)_{\mathrm{H}}+\left(\frac{\partial \mathrm{I}}{\partial f}\right)_{\mathrm{H}, e, g}\left(\frac{\partial f}{\partial T}\right)_{\mathrm{H}}+\left(\frac{\partial \mathrm{I}}{\partial g}\right)_{\mathrm{H}, e, f}\left(\frac{\partial g}{\partial \mathrm{T}}\right)_{\mathrm{H}} \\
& =\left(\frac{\partial \mathrm{I}}{\partial e}\right)_{\mathrm{H}, f, g}\left(\frac{\partial e}{\partial \mathrm{T}}\right)_{\mathrm{H}}+2\left(\frac{\partial \mathrm{I}}{\partial f}\right)_{\mathrm{H}, e, g}\left(\frac{\partial f}{\partial \mathrm{T}}\right)_{\mathrm{H}}, \cdots \cdot \cdot \cdot(3)
\end{aligned}
$$

since we may put $f=g$.

If $\mathrm{H}$ is zero, we have

$$
\frac{\partial e}{\partial \mathrm{T}}=\frac{m}{n(3 m-n)}, \quad \frac{\partial f}{\partial \mathrm{T}}=-\frac{m-n}{2 n(3 m-n)} ;
$$

hence, neglecting the change of elastic constants by magnetization, we have, from (3),

$$
\left(\frac{\partial \mathrm{I}}{\partial \mathrm{T}}\right)_{\mathrm{H}}=\frac{m}{n(3 m-n)}\left(\frac{\partial \mathrm{I}}{\partial e}\right)_{\mathrm{H}_{2}, g}-\frac{m-n}{n(3 m-n)}\left(\frac{\partial \mathrm{I}}{\partial f}\right)_{\mathrm{H}, e, g} .
$$

Hence, by (2) and (4), we get

But

$$
\frac{\partial e}{\partial \mathrm{I}}\left(\frac{\partial \mathrm{I}}{\partial \mathrm{H}}\right)_{e, f, g}=\left(\frac{\partial \mathrm{I}}{\partial \mathrm{T}}\right)_{\mathrm{H}} .
$$

$$
\begin{gathered}
\left(\frac{\partial \mathrm{I}}{\partial \mathrm{H}}\right)_{\mathrm{T}}=\left(\frac{\partial \mathrm{I}}{\partial \mathrm{H}}\right)_{e, f, g}+\left(\frac{\partial \mathrm{I}}{\partial e}\right)_{\mathrm{H}, f, g}\left(\frac{\partial e}{\partial \mathrm{H}}\right)_{\mathrm{T}}+\left(\frac{\partial \mathrm{I}}{\partial f}\right)_{\mathrm{H}, e, g}\left(\frac{\partial f}{\partial \mathrm{H}}\right)_{\mathrm{T}} \\
+\left(\frac{\partial \mathrm{I}}{\partial g}\right)_{\mathrm{H}, e, f}\left(\frac{\partial g}{\partial \mathrm{H}}\right)_{\mathrm{T}} \fallingdotseq\left(\frac{\partial \mathrm{I}}{\partial \ddot{\mathrm{H}}}\right)_{e, f, g} ;
\end{gathered}
$$

hence finally we get

$$
\left(\frac{\partial e}{\partial \mathrm{H}}\right)_{\mathrm{T}}=\left(\frac{\partial \mathrm{I}}{\partial \mathrm{T}}\right)_{\mathrm{H}} \cdot \ldots \cdot \cdot \cdot \cdot \cdot
$$

As to the twist, J. J. Thomson obtained a relation which, strictly speaking, holds in the case of a thin tube, i.e.,

$$
{ }_{n} \frac{\partial c}{\partial \mathrm{I}^{2}}=\frac{1}{2 \kappa}\left(\frac{\partial \mathrm{I}}{\partial c}\right)_{\mathrm{H}}\left\{1-\mathrm{H}\left(\frac{\partial \kappa}{\partial \mathrm{I}}\right)_{c}\right\} . .
$$

Here again in his original work, the factor $\frac{1}{2}$ is dropped ; $c$ is not the twist $\tau$ per unit-length, but it is connected with $\tau$ by the relation $c=r \tau$, where $r$ is the radius of the thin tube. As in the former case, we have

$$
1-\mathrm{H}\left(\frac{\partial \kappa}{\partial \mathrm{I}}\right)_{c}=\frac{\kappa}{\left(\frac{\partial \mathrm{I}}{\partial \mathrm{H}}\right)_{c}} \text { and }\left(\frac{\partial \mathrm{I}}{\partial \mathrm{H}}\right)_{c}=\left(\frac{\partial \mathrm{I}}{\partial \mathrm{H}}\right)_{\mathrm{L}} \text {, }
$$


in which $\mathrm{L}$ is the twisting couple. Equation (6) then becomes

$$
\frac{\partial c}{\partial \mathrm{I}}\left(\frac{\partial \mathrm{I}}{\partial \mathrm{H}}\right)_{\mathrm{x}}=\left(\frac{\partial c}{\partial \mathrm{H}}\right)_{\mathrm{L}}=\frac{1}{n}\left(\frac{\partial \mathrm{I}}{\partial c}\right)_{\mathrm{H}}
$$

or, very nearly,

$$
r^{2}\left(\frac{\partial \tau}{\partial \mathrm{H}}\right)_{\mathrm{L}}=\frac{1}{n}\left(\frac{\partial \mathrm{I}}{\partial \tau}\right)_{\mathrm{H}} \cdot
$$

The last equation, if it be integrated over the cross-section of the wire of radius $R$ gives

$$
\frac{\pi}{2} \mathrm{R}^{4}\left(\frac{\partial \tau}{\partial \mathrm{H}}\right)_{\mathrm{L}}=\frac{1}{n}\left(\frac{\partial}{\partial \tau} \int 2 \pi r \mathrm{I} d r\right)_{\mathrm{H}}=\frac{\pi \mathrm{R}^{2}}{n}\left(\frac{\partial \mathrm{I}_{m}}{\partial \tau}\right)_{\mathrm{H}^{\prime}}
$$

where $I_{m}$ is the mean intensity of magnetization. Hence finally

$$
\left(\frac{\partial \tau}{\partial \mathrm{H}}\right)_{\mathrm{L}}=\frac{2}{n \mathrm{R}^{2}}\left(\frac{\partial \mathrm{I}_{m}}{\partial \tau}\right)_{\mathrm{H}} \cdot \ldots . .
$$

From a thermodynamical consideration, A. Heydweiller obtained two relations, neglecting small quantities,

$$
\begin{aligned}
\frac{\partial e}{\partial \mathrm{H}} & =\frac{\partial \mathrm{I}}{\partial \mathrm{T}}+\frac{\mathrm{I}(1-2 \sigma)}{\mathrm{E}}, \quad . \quad . \quad . \quad . . \\
\frac{1}{\mathrm{E}^{2}} \frac{\partial \mathrm{E}^{\prime}}{\partial \mathrm{H}} & =-\frac{\partial^{2} \mathrm{I}}{\partial \mathrm{T}^{2}}-\frac{1-2 \sigma}{\mathrm{E}} \frac{\partial \mathrm{I}}{\partial \mathrm{T}}, \quad . \quad . \quad .
\end{aligned}
$$

where $\mathrm{E}$ is the modulus of elasticity, and $\sigma$ the Poisson ratio. In his original paper, $\sigma$ was put equal to $\frac{1}{3}$. Equation (9) was obtained by differentiating equation (8), considering $\sigma$ and $\mathrm{E}$ to be constant. But in magnetic fields, both $\sigma$ and $\mathrm{E}$ vary considerably with tension, as is shown by our previous experiment, so that if we retain the second term in the righthand side of equation (9), the term $\mathrm{I} \frac{\partial}{\partial T}\left(\frac{1-2 \sigma}{\mathrm{E}}\right)$ must be subtracted from it. But these terms being small compared with the first term, they may be neglected without causing any considerable error. The second term in equation (8) is also very small.

On another occasion*, Heydweiller gave relations which are very nearly equal to the last two with the second terms suppressed, and remarked that they are correct. Heydweiller's equation (8) differs from that given by $\mathbf{J}$. J. Thomson by a term of second importance.

* Rensing, loc. cit. p. 377. 
Rensing experimentally tested relation (9) in the case of iron and nickel, and showed a fair agreement between the theory and the experiment.

R. Gans criticised Heydweiller's equations and proposed his own, i.e.,

$$
\frac{\partial e}{\partial \mathrm{H}}=\frac{1}{4 \pi} \frac{\partial \mathrm{B}}{\partial \mathrm{T}}+\frac{\left(\mu-\mu_{0}\right)}{4 \pi \mathrm{E}} \mathrm{H}\left(\frac{1}{\mu_{0}} \frac{\partial \mathrm{B}}{\partial \mathrm{H}}-2 \sigma\right) .
$$

If the medium surrounding the magnet be air, we may put $\mu_{0}=1$; hence

$$
\frac{\partial e}{\partial \mathrm{H}}=\frac{\partial \mathrm{I}}{\partial \mathrm{T}}+\frac{\mathrm{I}(1-2 \sigma)}{\mathbf{E}}+\frac{2 \pi}{\mathrm{E}} \frac{\partial \mathrm{I}^{2}}{\partial \mathrm{H}} \cdot \ldots .
$$

Thus, Gans's equation differs from that of Heydweiller by the term $\frac{2 \pi}{\mathrm{E}} \frac{\partial \mathrm{I}^{2}}{\partial \mathrm{H}}$, which generally is not very small, but in weak fields it sometimes overweighs the first term. As in the case of Heydweiller, differentiating the above relation with respect to ' $\mathrm{l}$, Gans obtained an expression for the change of elasticity which differs from that of Heydweiller by the term $-\frac{2 \pi}{\mathrm{E}} \frac{\partial^{2} \mathrm{I}^{2}}{\partial^{\prime} \mathrm{T} \partial \mathrm{H}}$. Here again, it was assumed that $\sigma$ and $\mathrm{E}$ are independent of $\mathrm{T}$, a supposition not admissible in a magnetized wire.

By a similar consideration as Heydweiller, A. Kolâcêk obtained equation (8), and also a relation between magnetism and twist, i. e.,

$$
\frac{\partial \tau}{\partial H}=s \frac{\partial I}{\partial L}, \quad . \quad . \quad . \quad . .
$$

where $s$ is the cross-section of the wire. Since

$$
\mathrm{L}=\frac{\pi}{2} \mathrm{R}^{4} \tau n,
$$

the above equation becomes

$$
\frac{\partial \tau}{\partial \mathrm{H}}=\frac{2}{n \mathrm{R}^{2}} \frac{\partial \mathrm{I}}{\partial \tau}, \quad \cdot \quad \cdot \quad \cdot
$$

which coincides with equation (9).

M. Cantone obtained two relations by equating the change of magnetic energy due to a tension or a twist to the change of elastic energy caused by magnetization, i. e.,

$$
e_{m}=\frac{\partial}{\partial \tau} \int_{0}^{\mathrm{I}} \mathrm{H} d \mathrm{I} \text { and } \tau_{m}=s \frac{\partial}{\partial \mathrm{L}} \int_{0}^{\mathrm{I}} \mathrm{H} d \mathrm{I}
$$


whore $e_{m}$ and $\tau_{m}$ are the magnetic strains. By differentiating the above equations with respect. to $H$, we have

$$
\begin{aligned}
& \frac{\partial e}{\partial \mathrm{H}}=\frac{\partial}{\partial \mathrm{T}}\left(\mathrm{H} \frac{\partial \mathrm{I}}{\partial \mathrm{H}}\right), \ldots . \\
& \frac{\partial \tau}{\partial \mathrm{H}}=s \frac{\partial}{\partial \mathrm{L}}\left(\mathrm{H} \frac{\partial \mathrm{I}}{\partial \mathrm{H}}\right) .
\end{aligned}
$$

Cantone tested the second relation by experiment and found a satisfactory agreement in iron and nickel. For the first relation, he also made a comparison between theory and experiment, but the data he used were taken from experiments by different physicists, so that they do not refer to the same specimen. Though the comparison shows a satisfactory agreement, it is not certain whether it was by chance or not.

By a direct method, Dr. S. Sano obtained the relation

$$
\frac{\partial e}{\partial \mathrm{H}}=\frac{\partial \mathrm{I}}{\partial \mathrm{T}}+\frac{\mathrm{I}(1-2 \sigma)}{\mathrm{H}}+\frac{2 \pi \partial\left(\kappa_{0}{ }^{2} \mathrm{H}^{2}\right)}{\partial \mathrm{H}}, \ldots
$$

where $\kappa_{0}$ is a term in the expression of susceptibility, which is independent of the strain. Since $\mathrm{I} \leftrightharpoons \kappa_{0} \mathrm{H}$, Sano's equation practically coincides with Gans's. For the change of elasticity, Dr. Sano obtained

$$
\frac{1}{\mathrm{~L}^{2}} \frac{\partial \mathrm{E}}{\partial \mathrm{H}}=-\frac{\partial^{2} \mathrm{I}}{\partial \mathrm{T}^{2}}, \quad \cdot \quad \cdot \cdot \cdot \cdot
$$

which is practically the same as Heydweiller's equation, but different from Gans's by a term not negligibly small in weak fields. The above equation was obtained independently of the relation for $\frac{\partial e}{\partial \mathrm{H}}$. As to the effect of twist, Dr. Sano obtained an equation which can be transformed into (12).

Thus far, the relations for $\frac{\partial e}{\partial \mathrm{H}}$ given by Heydweiller, Gans, Kolâcêk, and Sano all agree with one another in the first important term $\frac{\partial \mathrm{L}}{\partial \mathrm{T}}$. Relation (1) given by J. J. Thomson does not differ in reality from others. Relation (13) given by Cantone also coincides with others in the first term, provided $\kappa$ is independent of $H$. The sesond term $I(1-2 \sigma) / \mathrm{E}$ in (8) and (10) may be neglected for the first 
approximation; the third term in relation (10), which becomes important in weak fields, is properly to be added.

The relations for $\frac{\partial \mathrm{E}}{\partial \mathrm{H}}$ given by Heydweiller, Gans, and Sano also agree with each other in the first term $-\frac{\partial^{2} \mathrm{I}}{\partial \mathrm{T}^{2}}$. Gans's differs principallv from the others by a term not generally small in weak fields.

As regards the relation for $\frac{\partial \tau}{\partial H}$, Kolâcêk's and Sano's coincide with each other. Thomson's relation (6) also does not differ from the others. If $\boldsymbol{\kappa}$ be independent of $\mathrm{H}$, Cantone's formula coincides with the others.

Thus, the chicf relations to be tested by experiments are as follows :-

$$
\begin{aligned}
& \frac{\partial e}{\partial \mathrm{H}}=\frac{\partial \mathrm{I}}{\partial^{\prime} \mathrm{I}}+\frac{2 \pi}{\mathrm{E}} \frac{\partial \mathrm{I}^{2}}{\partial \mathrm{H}}, \\
& \frac{1}{\overline{\mathrm{E}}^{2}} \frac{\partial \mathrm{E}}{\partial \mathrm{H}}=-\frac{\partial^{2} \mathrm{I}}{\partial \mathrm{T}^{2}}, \\
& \frac{\partial \tau}{\partial \mathrm{H}}=\frac{2}{n \overline{\mathrm{R}}^{2}} \frac{\partial \mathrm{I}}{\partial \tau}=s \frac{\partial \mathrm{I}}{\partial \mathrm{L}} .
\end{aligned}
$$

Our present experiments combined with the previous investigations on the change of elastic property due to magnetization furnish us with good materials for the testing of these relations.

Our results taken as a whole, give for the effect of tension as well as of twist two different sets of experimental data corresponding to the different orders of applying the magnetic field and the stress. The mutual correspondence of the results in the previous and the present experiments in this respect is tabulated below :-

Change of Strains by Magnetization.

1. Magnetic elongation under constant tension.

Elongation by tension under constant field.

2. Magnetic twisting under constant couple (Barus's method).

Change of rigidity under constant field (oscillation method).
Change of Magnetization by Strain.

1'. Magnetization under constant tension.

Change of magnetization by tension under constant field.

2'. Magnetization under constant twist.

Change of magnetization by twist under constant field. 
The theoretical relations to be tested were, however, deduced on the supposition that the magnetization is independent of the order of magnetizing and straining, so that in comparing the theory and the experiment, too much weight is not to be placed on the above correspondence.

The values of $\frac{\partial e}{\partial H^{\prime}}, \frac{\partial \mathrm{E}}{\partial \mathrm{H}}$ and $\frac{\partial \tau}{\partial \mathrm{H}}$ were deduced from our previous experiment, while the corresponding values for $\frac{\partial I}{\partial T^{T}}, \frac{\partial^{2} \mathrm{I}}{\partial T^{2}}$, and $\frac{\partial \mathrm{I}}{\partial \mathrm{L}}$ were obtained from the present experiment. In the following tables, $\left[\frac{\partial \mathrm{I}}{\partial \mathrm{T}}\right]_{\mathrm{H}}$ and $\left[\frac{\partial^{2} \mathrm{~T}}{\partial \mathrm{T}^{2}}\right]_{\mathrm{H}}$ are the values of these differential coefficients obtained from curves $(\delta I, T)_{\mathrm{H}}$; while $\left[\frac{\partial \mathrm{I}}{\partial T}\right]_{\mathrm{T}}$ and $\left[\frac{\partial^{2} \mathrm{I}}{\partial^{\prime} \mathrm{T}^{2}}\right]_{\mathrm{T}}$ are the values of the coefficients from curves $(\mathrm{I}, \mathrm{H})_{\mathrm{T}} \cdot \frac{1}{\overline{\mathrm{E}^{2}}}\left[\frac{\partial \mathrm{E}}{\partial \mathrm{H}}\right]_{\mathrm{H}}$ is the value obtained from the results of the elongation method; while $\frac{1}{\mathrm{E}^{2}}\left[\frac{\partial \mathrm{E}}{\partial \mathrm{H}}\right]_{\mathbf{T}}$ is that obtained from the tension effect on magnetic elongation. $\frac{1}{\tau}\left[\frac{\partial \tau}{\partial \mathrm{H}}\right]_{\mathrm{H}}$ is the value of the coefficient obtained from the result of the oseillation method, and $\frac{1}{\tau}\left[\frac{\partial \tau}{\partial H}\right]_{r}$ that obtained from Barus's method; while $\frac{s}{\tau}\left[\frac{\partial \mathrm{I}}{\partial \mathrm{L}}\right]_{\mathrm{H}}$ and $\frac{s}{\tau}\left[\frac{\partial \mathrm{I}}{\partial \mathrm{L}}\right]_{\tau}$ are the values obtained from curves $(\delta \mathrm{I}, \tau)_{\mathrm{H}}$ and $(\mathrm{I}, \mathrm{H})_{\tau}$ respectively. To find the values of these differential coefficients, corresponding curves were carefully drawn on section papers ; a fine straight line drawn on a thin glass plate was brought into contact with the curve at the required point and the trigonometrical tangent of the inclination of this straight line was evaluated. Some of the calculated results are given below :- 
Swedish Iron.

\begin{tabular}{|c|c|c|c|c|c|c|c|}
\hline \multirow[b]{2}{*}{ H. } & \multicolumn{4}{|c|}{$\mathrm{T}=1627 \mathrm{gr} . / \mathrm{mm} .2$} & \multicolumn{3}{|c|}{$\mathrm{I}^{\prime}=5535 \mathrm{gr} . / \mathrm{mm}}$. \\
\hline & $\frac{\partial e}{\partial \ddot{H}}$ & {$\left[\frac{\partial I}{\partial^{\prime} \mathrm{T}}\right] \mid$} & {$\left[\frac{\partial I}{\partial T}\right]_{T}$} & $\left|\begin{array}{c}2 \pi \\
\mathrm{E} \frac{\mathrm{I}^{2}}{\partial \mathrm{H}}\end{array}\right|$ & $\frac{\partial e}{\partial \mathrm{H}}$ & {$\left[\frac{\partial I}{\partial^{\prime} \mathrm{I}}\right]_{\mathrm{II}}$} & {$\left[\begin{array}{l}\partial \mathrm{I} \\
\partial^{\prime} \hat{\bar{I}}\end{array}\right]_{T}$} \\
\hline & \multicolumn{7}{|c|}{ Measured in $10^{-8}$. } \\
\hline $\begin{array}{c}5 \cdot 9 \\
11 \cdot 9 \\
24 \cdot 2 \\
36 \cdot 6 \\
97 \cdot 1 \\
207 \\
367\end{array}$ & $\begin{array}{l}+18 \\
+14 \\
+\quad 0.8 \\
-0.7 \\
-1.8 \\
-2.0 \\
-1.4\end{array}$ & $\left|\begin{array}{l}+72 \\
+13 \\
+0.6 \\
-1.7 \\
-2.4 \\
-2.2 \\
-1.7\end{array}\right|$ & $\begin{array}{c}+63 \\
+12 \\
+3 \cdot 3 \\
+2 \cdot 0 \\
\ldots \\
\ldots \\
\ldots\end{array}$ & $\begin{array}{l}+33 \\
+14 \\
+\quad 4.7 \\
+2.7 \\
+1.2 \\
+0.7 \\
+0.4\end{array}$ & $\begin{array}{l}+1.0 \\
+0.7 \\
-1.0 \\
-1.7 \\
-2.1 \\
-1.9 \\
-1.4\end{array}$ & $\begin{array}{l}+20 \\
+2 \cdot 0 \\
-0.8 \\
-1.9 \\
-2 \cdot 0 \\
-2.3 \\
-1.9\end{array}$ & $\begin{array}{l}+0.0 \\
+0.0 \\
-7 \cdot 0 \\
-6 \cdot 2 \\
-5 \cdot 0 \\
-8.0 \\
\ldots\end{array}$ \\
\hline \multicolumn{8}{|c|}{$\mathrm{T}=4754 \mathrm{gr} . / \mathrm{mm} .2$} \\
\hline \multirow[t]{2}{*}{ H. } & \multicolumn{2}{|c|}{$-\frac{1}{\mathbf{E}^{2}}\left[\frac{\partial \mathrm{E}}{\partial \mathrm{H}}\right]$} & & \multicolumn{2}{|c|}{$\left[\frac{\partial^{2} \mathrm{I}}{\partial^{\prime} \mathrm{I}^{\prime 2}}\right]_{\mathrm{H}}$} & \multicolumn{2}{|c|}{$\left[\frac{\partial^{2} \mathbf{I}}{\partial^{\prime} \mathbf{I}^{2}}\right]_{\mathbf{T}}$} \\
\hline & \multicolumn{7}{|c|}{ Measured in $10^{-17}$. } \\
\hline $\begin{array}{r}5 \cdot 9 \\
11 \cdot 9 \\
24 \cdot 2 \\
36 \cdot 6 \\
97 \cdot 1 \\
207 \\
367\end{array}$ & & $\begin{array}{l}-19 \\
-19 \\
-\quad 8.8 \\
+\quad 3 \cdot 3 \\
+\quad 0.05 \\
+\quad 0.03 \\
+\quad 0.00\end{array}$ & & $\begin{array}{r}-29 \\
-2.8 \\
-1.0 \\
-\quad 06 \\
-0.3\end{array}$ & & $\begin{array}{c}\dddot{4} \\
\ldots \\
-1 \\
\ldots \\
-3 \\
\ldots\end{array}$ & \\
\hline
\end{tabular}

$$
\mathrm{T}=3255 \mathrm{gr} . / \mathrm{mm}^{2} .
$$

\begin{tabular}{|c|c|c|c|c|}
\hline & & $\tau=6^{\prime} \cdot 3$ & $\tau=6^{\prime} 3$ & $r=6^{\prime} \cdot 3$ \\
\hline \multirow[t]{2}{*}{ H. } & $\frac{1}{\tau}\left[\frac{\partial \tau}{\partial H}\right]_{\mathrm{II}}$ & $\frac{1}{\tau}\left[\begin{array}{l}\partial \tau \\
\partial \ddot{H}\end{array}\right]_{\tau}$ & $\stackrel{s}{\tau}\left[\begin{array}{l}\partial \mathrm{I} \\
\partial \overline{\mathrm{L}}\end{array}\right]_{\mathrm{II}}$ & $\frac{s}{r}\left[\frac{\partial I}{\partial L}\right]_{\tau}$ \\
\hline & \multicolumn{4}{|c|}{ Measured in $10^{-4}$. } \\
\hline 4 & ... & -11 & +128 & +64 \\
\hline 6 & $\ldots$ & -13 & +92 & -80 \\
\hline 8 & & -11 & +48 & -48 \\
\hline 14 & $+0 \cdot 23$ & -36 & +24 & $-1 \cdot 6$ \\
\hline $2 \%$ & -0.15 & -27 & +12 & $\ldots$ \\
\hline 50 & -0.40 & $-1 \cdot 1$ & $+\quad 30$ & $\ldots$ \\
\hline 110 & -0.40 & -0.33 & +0.7 & .. \\
\hline 200 & +0.12 & $-0 \cdot 11$ & +0.0 & $\ldots$ \\
\hline 300 & +007 & -0.07 & $\cdots$ & ... \\
\hline
\end{tabular}




\section{Tungsten-Steel.}

\begin{tabular}{|c|c|c|c|c|c|c|c|c|}
\hline \multirow[b]{2}{*}{ H. } & \multicolumn{5}{|c|}{$\mathrm{T}=1693 \mathrm{gr} . / \mathrm{mm} .^{2}$} & \multicolumn{3}{|c|}{$' \Gamma=5762 \mathrm{grc} / \mathrm{mm}_{.2}{ }^{2}$} \\
\hline & $\frac{\partial c}{\partial \mathrm{H}}$ & {$\left[\frac{\partial I}{\partial T}\right]$} & & {$\left[\frac{\partial \mathrm{I}}{\partial \mathrm{T}}\right]_{\mathrm{T}}$} & $\frac{2 \pi}{\mathrm{E}} \frac{\partial \mathrm{I}^{2}}{\partial \mathrm{H}}$ & $\frac{\partial e}{\partial \mathbf{H}}$ & {$\left[\frac{\partial \mathrm{I}}{\partial \mathrm{I}}\right]$} & {$\left[\frac{\dot{\partial I}}{\partial{ }^{\prime}}\right]_{T}$} \\
\hline & \multicolumn{8}{|c|}{ Measured in $10^{-8}$. } \\
\hline $\begin{array}{c}10 \cdot 6 \\
23 \cdot 9 \\
43 \cdot 4 \\
98 \cdot 4 \\
210 \\
341\end{array}$ & $\begin{array}{l}+0.9 \\
+1.2 \\
+3.4 \\
+0.57 \\
-0.10 \\
-0.25\end{array}$ & \multicolumn{2}{|c|}{$\begin{array}{l}+3.3 \\
+23.0 \\
+4.3 \\
+0.38 \\
-0.32 \\
-0.32\end{array}$} & $\begin{array}{l}+0.0 \\
+24 \\
+0.0 \\
-2.9 \\
-30 \\
-31\end{array}$ & $\begin{array}{l}+0.65 \\
+20.6 \\
+4.8 \\
+1.2 \\
+0.50 \\
+0.23\end{array}$ & $\begin{array}{l}+0.7 \\
+0.5 \\
+5.5 \\
+0.35 \\
-0.25 \\
-0.29\end{array}$ & $\begin{array}{l}+4.6 \\
+18 \\
+4.4 \\
+0.40 \\
-0.38 \\
-0.36\end{array}$ & $\begin{array}{l}+00 \\
+13 \\
+0.0 \\
-50 \\
-3 \cdot 6 \\
-36\end{array}$ \\
\hline \multirow[b]{2}{*}{ H. } & \multicolumn{8}{|c|}{$\mathrm{T}=4947 \mathrm{gr} . / \mathrm{mm} .^{2}$} \\
\hline & \multicolumn{2}{|c|}{$-\frac{1}{\mathbf{E}^{2}}\left[\frac{\partial \mathrm{E}}{\partial \bar{H}}\right]_{11}$} & \multicolumn{2}{|c|}{$-\frac{1}{\mathbf{E}^{2}}\left[\frac{\partial \mathrm{H}}{\partial H}\right]$} & ]$_{\mathbf{T}}$ & $\left.\frac{\partial^{2 t}}{\partial \mathrm{T}^{2}}\right]_{\mathrm{I}}$ & \multicolumn{2}{|c|}{$\left[\frac{\partial^{2} I}{\partial^{\prime} I^{2}}\right]_{T}$} \\
\hline & \multicolumn{8}{|c|}{ Measured in $10^{-17}$. } \\
\hline $\begin{array}{c}23 \cdot 9 \\
43 \cdot 4 \\
98 \cdot 4 \\
210 \\
341\end{array}$ & \multicolumn{2}{|c|}{$\begin{array}{l}-3.6 \\
-2.1 \\
-1.8 \\
-0.0 \\
+0.41\end{array}$} & & $\begin{array}{l}-4.1 \\
-3.5 \\
-0.83 \\
-0.32 \\
-0.17\end{array}$ & & $\begin{array}{l}-18 \\
-6.2 \\
-0.0 \\
-0.0 \\
-0.0\end{array}$ & \multicolumn{2}{|c|}{$\begin{array}{r}-43 \\
0.0 \\
+\quad 1.2 \\
+\quad 42 \\
0.0\end{array}$} \\
\hline
\end{tabular}

$\mathrm{T}=3340 \mathrm{gr} \cdot / \mathrm{mm}^{2}$.

\begin{tabular}{|c|c|c|c|}
\hline & & $\tau=20^{\prime} \cdot 0$ & $\tau=20^{\prime} \cdot 0$ \\
\hline \multirow[t]{2}{*}{ H. } & $\frac{1}{\tau}\left[\begin{array}{l}\partial \tau \\
\partial H\end{array}\right]_{I I}$ & $\frac{1}{\tau}\left[\begin{array}{c}\partial \tau \\
\partial \bar{H}\end{array}\right]_{\tau}$ & $\stackrel{s}{\tau}\left[\begin{array}{l}\partial \mathrm{I} \\
\partial \mathrm{L}\end{array}\right]_{\mathrm{H}}$ \\
\hline & \multicolumn{3}{|c|}{ Measured in $10^{-4}$} \\
\hline $\begin{array}{l}10 \cdot 7 \\
18 \cdot 4 \\
30 \cdot 1 \\
98 \cdot 8 \\
364\end{array}$ & $\begin{array}{l}+0.23 \\
+0.10 \\
-0.04 \\
-0.03 \\
-0.03\end{array}$ & $\begin{array}{c}-\ddot{1} \cdot 0 \\
-0.53 \\
-0.15 \\
-0.03\end{array}$ & $\begin{array}{l}+1.5 \\
+7.9 \\
+3.2 \\
+0.3 \\
-0.04\end{array}$ \\
\hline
\end{tabular}


Nickel.

\begin{tabular}{|c|c|c|c|c|c|c|c|c|}
\hline \multirow[b]{2}{*}{ H. } & \multicolumn{5}{|c|}{$\mathrm{T}=1540 \mathrm{gr} . / \mathrm{mm}^{2}$} & \multicolumn{3}{|c|}{$\mathrm{T}=5240 \mathrm{gr} . / \mathrm{mm}^{2}$} \\
\hline & $\frac{\partial e}{\partial H}$ & {$\left[\frac{\partial \mathfrak{I}}{\partial T}\right]_{\mathrm{H}}$} & {$\left[\frac{\partial \mathrm{I}}{\partial^{\prime} \mathrm{T}}\right]_{\mathbf{T}}$} & \multicolumn{2}{|c|}{$\frac{2 \pi \partial \mathrm{I}^{2}}{\mathrm{E} \partial \overline{\mathrm{H}}}$} & $\frac{\partial e}{\partial H}$ & {$\left[\frac{\partial I}{\partial T}\right]_{H}$} & {$\left[\frac{\partial I}{\partial T}\right]_{T}$} \\
\hline \multirow[b]{2}{*}{$\begin{array}{l}10 \cdot 8 \\
31 \cdot 1 \\
62 \cdot 5 \\
135 \\
206 \\
\mathbf{3 6 5}\end{array}$} & \multicolumn{8}{|c|}{ Measured in $10^{-7}$. } \\
\hline & $\begin{array}{l}-5.3 \\
-7.7 \\
-2.2 \\
-0.68 \\
-0.32 \\
-0.05\end{array}$ & $\begin{array}{l}-3 \\
-3 \\
-2 \\
-0 \\
-0 \\
-0\end{array}$ & $\begin{array}{l}-5.1 \\
-4.9 \\
-2.8 \\
-0.66 \\
-0.38 \\
-0.05\end{array}$ & $\begin{array}{l}+0 \\
+0 \\
+0 \\
+0 \\
+0 \\
+0\end{array}$ & & $\begin{array}{l}-0.40 \\
-1.8 \\
-3.1 \\
-2.0 \\
-0.80 \\
-0.17\end{array}$ & $\begin{array}{l}-2.2 \\
-3.8 \\
-3.5 \\
-1.6 \\
-0.72 \\
-0.17\end{array}$ & $\begin{array}{l}-3.9 \\
-3.2 \\
-3 \cdot 1 \\
-1.8 \\
-1 \cdot 1 \\
-0.3\end{array}$ \\
\hline & \multicolumn{8}{|c|}{$\mathrm{T}=4498 \mathrm{gr} . / \mathrm{mm}^{2}{ }^{2}$} \\
\hline H. & \multicolumn{2}{|c|}{$-\frac{1}{\mathrm{E}^{2}}\left[\begin{array}{l}\partial \mathrm{E} \\
\partial \mathrm{H}\end{array}\right]_{\mathrm{H}}$} & \multicolumn{2}{|c|}{$-\frac{1}{\mathrm{E}^{2}}\left[\frac{\partial \mathrm{E}}{\partial \mathrm{H}}\right]_{\mathrm{T}}$} & \multicolumn{2}{|c|}{$\left[\begin{array}{l}\partial^{2} I \\
\partial T^{2}\end{array}\right]_{H}$} & \multicolumn{2}{|c|}{$\left[\frac{\partial^{2} T}{\partial T^{2}}\right]_{T}$} \\
\hline \multirow[b]{2}{*}{$\begin{array}{l}10 \cdot 8 \\
31 \cdot 7 \\
62 \cdot 5 \\
135 \\
206 \\
365\end{array}$} & \multicolumn{8}{|c|}{ Measured in $10^{-16}$} \\
\hline & \multicolumn{2}{|c|}{$\begin{array}{l}+2 \cdot 3 \\
+3 \cdot 7 \\
+7 \cdot 6 \\
-3 \cdot 3 \\
-1 \cdot 2 \\
-0.44\end{array}$} & \multicolumn{2}{|c|}{$\begin{array}{l}+2.3 \\
+4 \cdot 2 \\
+13.7 \\
-5.2 \\
-1.3 \\
--0.55\end{array}$} & \multicolumn{2}{|c|}{$\begin{array}{l}+2.0 \\
+8.9 \\
-2.6 \\
-3.6 \\
-0.85 \\
-0.39\end{array}$} & \multicolumn{2}{|c|}{$\begin{array}{r}+0.0 \\
+15.3 \\
+7 \cdot 3 \\
+4 \cdot 1 \\
+3.4 \\
+5.1\end{array}$} \\
\hline \multirow{3}{*}{$\mathbf{T}$} & \multicolumn{8}{|c|}{$\mathrm{T}_{m}=3400 \mathrm{gr} . / \mathrm{mm} .^{2}$} \\
\hline & \multicolumn{2}{|c|}{$3410 \mathrm{gr}$. } & $3096 \mathrm{gr}$. & 1 & & $546 \mathrm{gr}$. & \multicolumn{2}{|c|}{$3546 \mathrm{gr}}$. \\
\hline & & & \multicolumn{6}{|c|}{$\tau=12^{\prime} \cdot 6$} \\
\hline \multirow[t]{2}{*}{ H. } & \multicolumn{2}{|c|}{$\frac{1}{\tau}\left[\frac{\partial \tau}{\partial \mathrm{H}}\right]_{\mathrm{II}}$} & $\frac{1}{\tau}\left[\frac{\partial r}{\partial H}\right]_{T}$ & & \multicolumn{2}{|c|}{$\frac{\varepsilon}{\tau}\left[\frac{\partial \mathrm{I}}{\partial \mathrm{L}}\right]_{\mathrm{H}}$} & \multicolumn{2}{|c|}{$\frac{s}{\tau}\left[\frac{\partial I}{\partial L}\right]_{T}$} \\
\hline & \multicolumn{8}{|c|}{ Measured in $10^{-3}$} \\
\hline $\begin{array}{r}2 \cdot 4 \\
4 \cdot 0 \\
106 \\
23.8 \\
49 \cdot 6 \\
97.0 \\
168 \\
358\end{array}$ & $\begin{array}{l}+0 \\
+0 \\
+0 \\
+1 \\
+1 \\
-0 \\
-0 \\
-0\end{array}$ & & $\begin{array}{l}-1.4 \\
-3.4 \\
+2.5 \\
+3.5 \\
+0.4 \\
-0.48 \\
-0.50 \\
-0.20\end{array}$ & & & $\begin{array}{l}+1 \cdot 9 \\
+3 \cdot 6 \\
+3.5 \\
+1 \cdot 9 \\
+0 \cdot 7 \\
+0 \cdot 78 \\
+0 \cdot 17 \\
+0.05\end{array}$ & & $\begin{array}{l}+0.30 \\
+3 \cdot 5 \\
+1.8 \\
+0.96 \\
-0.39 \\
-0.26 \\
\ldots\end{array}$ \\
\hline
\end{tabular}


Effect of Stress on Magnetization.

28.74 per cent. Nickel-Steel.

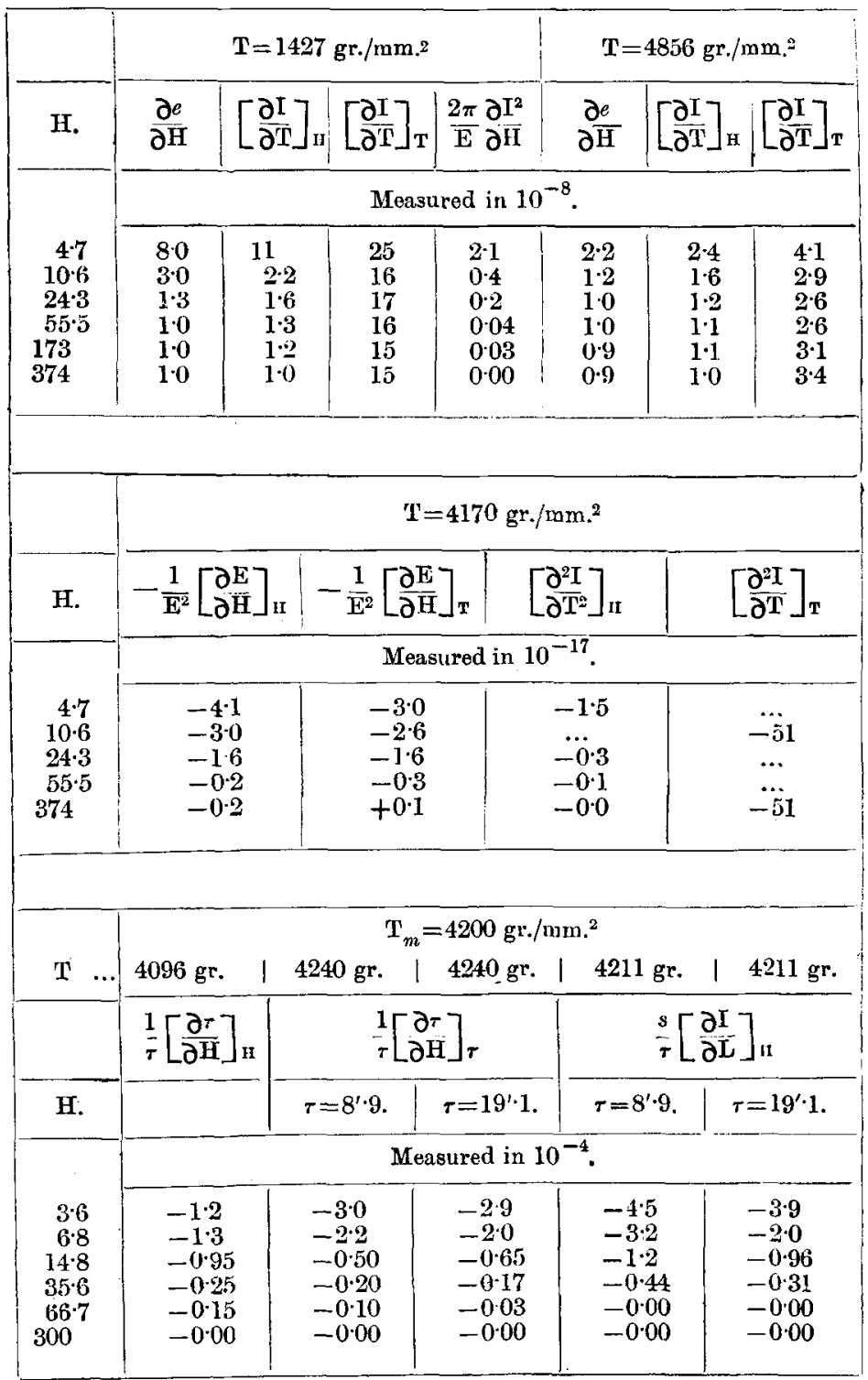


50.72 per cent. Nickel-Steel.

\begin{tabular}{|c|c|c|c|c|c|c|c|c|c|c|}
\hline & \multicolumn{5}{|c|}{$\mathrm{T}=890 \mathrm{gr} . / \mathrm{mm} .^{2}$} & \multicolumn{5}{|c|}{${ }^{*} \mathrm{~T}=6652 \mathrm{gr} . / \mathrm{mm} .^{2}$} \\
\hline H. & $\begin{array}{l}\partial e \\
\partial \dot{H}\end{array}$ & {$\left[\begin{array}{l}\partial I \\
\partial T\end{array}\right]_{H}$} & {$\left[\frac{\partial I}{\partial T}\right]_{T}$} & & $\frac{\partial I^{2}}{\partial H}$ & & $\frac{\partial e}{\mathrm{H}}$ & {$\left[\frac{\partial I}{\partial T}\right.$} & ]$_{\mathrm{in}}$ & {$\left[\frac{\partial 1}{\partial T}\right]_{T}$} \\
\hline & \multicolumn{10}{|c|}{ Measured in $10^{-7}$. } \\
\hline $\begin{array}{c}1 \cdot 9 \\
5 \cdot 5 \\
10^{\circ} 7 \\
23 \cdot 8 \\
51 \cdot 4 \\
151 \\
360\end{array}$ & $\begin{array}{c}11 \cdot 9 \\
6 \cdot 3 \\
3 \cdot 7 \\
1 \cdot 7 \\
0 \cdot 34 \\
0.04 \\
0.03\end{array}$ & $\begin{array}{l}20 \\
8 \cdot 2 \\
5 \cdot 7 \\
2 \cdot 2 \\
0 \cdot 61 \\
0 \cdot 03 \\
0 \cdot 0 .\end{array}$ & $\begin{array}{l}9 \cdot 0 \\
7 \cdot 1 \\
4 \cdot 0 \\
1 \cdot 9 \\
0 \cdot 70 \\
0 \cdot 32 \\
0 \cdot 25\end{array}$ & & $\begin{array}{l}1 \cdot 2 \\
2 \cdot 2 \\
1 \cdot 0 \\
0 \cdot 1 \\
0 \cdot 07 \\
0.01 \\
0 \cdot 01\end{array}$ & \multicolumn{2}{|c|}{$\begin{array}{l}-0.62 \\
-0.83 \\
-0.25 \\
-0.09 \\
-0.05 \\
-0.01 \\
-0.00\end{array}$} & \multicolumn{2}{|c|}{$\begin{array}{l}-0 \cdot 16 \\
-0 \cdot 16 \\
-0.08 \\
-0.05 \\
-0.02 \\
-0.01 \\
-0.00\end{array}$} & $\begin{array}{l}-0.78 \\
-0.42 \\
-0.57 \\
-0.51 \\
-0.51 \\
-0.51 \\
-0.36\end{array}$ \\
\hline 360 & \multicolumn{10}{|c|}{$\mathrm{T}=5003 \mathrm{gr} \cdot / \mathrm{mm} .^{2}$} \\
\hline \multirow[t]{2}{*}{ H. } & \multicolumn{2}{|c|}{$-\frac{1}{\mathrm{E}^{2}}\left[\frac{\partial \mathrm{E}}{\partial \mathrm{H}}\right]_{\mathrm{H}}$} & \multicolumn{2}{|c|}{$\frac{1}{\mathrm{E}^{2}}\left[\frac{\partial \mathrm{E}}{\partial \mathrm{H}}\right]_{\mathrm{T}}$} & \multicolumn{3}{|c|}{$\left[\frac{\partial^{2} \mathrm{I}}{\partial \mathrm{T}^{2}}\right]_{\mathrm{H}}$} & \multicolumn{3}{|c|}{$\left[\frac{\partial^{2} I}{\partial T^{2}}\right]_{\mathbf{T}}$} \\
\hline & \multicolumn{10}{|c|}{ Measured in $10^{-7}$. } \\
\hline $\begin{array}{r}5 \cdot 5 \\
10 \cdot 7 \\
23 \cdot 8 \\
51 \cdot 4 \\
151 \cdot 0 \\
360\end{array}$ & \multicolumn{2}{|c|}{$\begin{array}{l}-0.19 \\
-0.13 \\
-0.10 \\
-0.04 \\
-0.01 \\
-0.00\end{array}$} & \multicolumn{2}{|l|}{$\begin{array}{l}-0.20 \\
-0.15 \\
-0.09 \\
-0.03 \\
-0.01 \\
-0.00\end{array}$} & \multicolumn{3}{|c|}{$\begin{array}{l}-0.51 \\
-0.10 \\
-0.10 \\
-0.00 \\
-0.00 \\
-0.00\end{array}$} & \multicolumn{3}{|c|}{$\begin{array}{l}-0.66 \\
-0.56 \\
-0.36 \\
-0.41 \\
-0.34 \\
-0.23\end{array}$} \\
\hline \multirow[t]{2}{*}{$\mathbf{T}$} & \multicolumn{9}{|c|}{$\mathrm{T}_{m}=3400 \mathrm{gr} . / \mathrm{mm}^{2}{ }^{2}$} & $409 \mathrm{gr}$. \\
\hline & $\frac{1}{\tau}\left[\frac{\partial \tau}{\partial H}\right]_{\Pi}$ & & $\left.\frac{\partial \tau}{\vec{H}}\right]_{\tau}$ & & & {$\left[\frac{\partial}{\partial}\right.$} & {$\left[\begin{array}{l}1 \\
\mathrm{~L}\end{array}\right]_{\mathrm{II}}$} & & & {$\left[\frac{\partial I}{\partial L}\right]_{\tau}$} \\
\hline \multirow[t]{2}{*}{ H. } & & $\tau=6 \cdot 2^{\prime}$ & $\tau=18 \cdot 6$ & & $\tau=$ & & $\tau=$ & $2 \cdot 3$ & & $=17 \cdot 4^{\prime}$ \\
\hline & \multicolumn{10}{|c|}{ Measured in $10^{-3}$} \\
\hline $\begin{array}{r}4 \cdot 2 \\
10 \cdot 5 \\
24 \cdot 5 \\
49 \cdot 6 \\
109 \cdot 0 \\
212 \\
377\end{array}$ & $\begin{array}{l}-1.5 \\
-1.8 \\
-0.95 \\
-0.42 \\
-0.17 \\
-0.07 \\
-0.02\end{array}$ & $\begin{array}{l}-1 \cdot 8 \\
-1 \cdot 7 \\
--0 \\
-0 \cdot 47 \\
-0 \cdot-7 \\
-0.05 \\
-0.02\end{array}$ & $\begin{array}{l}-1.8 \\
-1.4 \\
-0.90 \\
-0.47 \\
-0.17 \\
-0.05 \\
-0.02\end{array}$ & & $\begin{array}{l}+9 \\
+5 \\
+2 \\
+1 \\
+0 \\
+0 \\
+0\end{array}$ & & $\begin{array}{l}-1 \\
-1 \\
-1 \\
-1 \\
- \\
- \\
-\end{array}$ & $\begin{array}{l}.2 \\
.1 \\
69 \\
35 \\
13 \\
04 \\
00\end{array}$ & & $\begin{array}{l}-1 \cdot 6 \\
-1.5 \\
-0.73 \\
-0.44 \\
-0.12 \\
-0.00 \\
-0.00\end{array}$ \\
\hline
\end{tabular}

* Under a high tension, this specimen slightly contracts by magnetization, which is also to be expected from the effect of tension on magnetization. 
Effect of Stress on Magnetization.

70.32 per cent. Nickel-Steel.

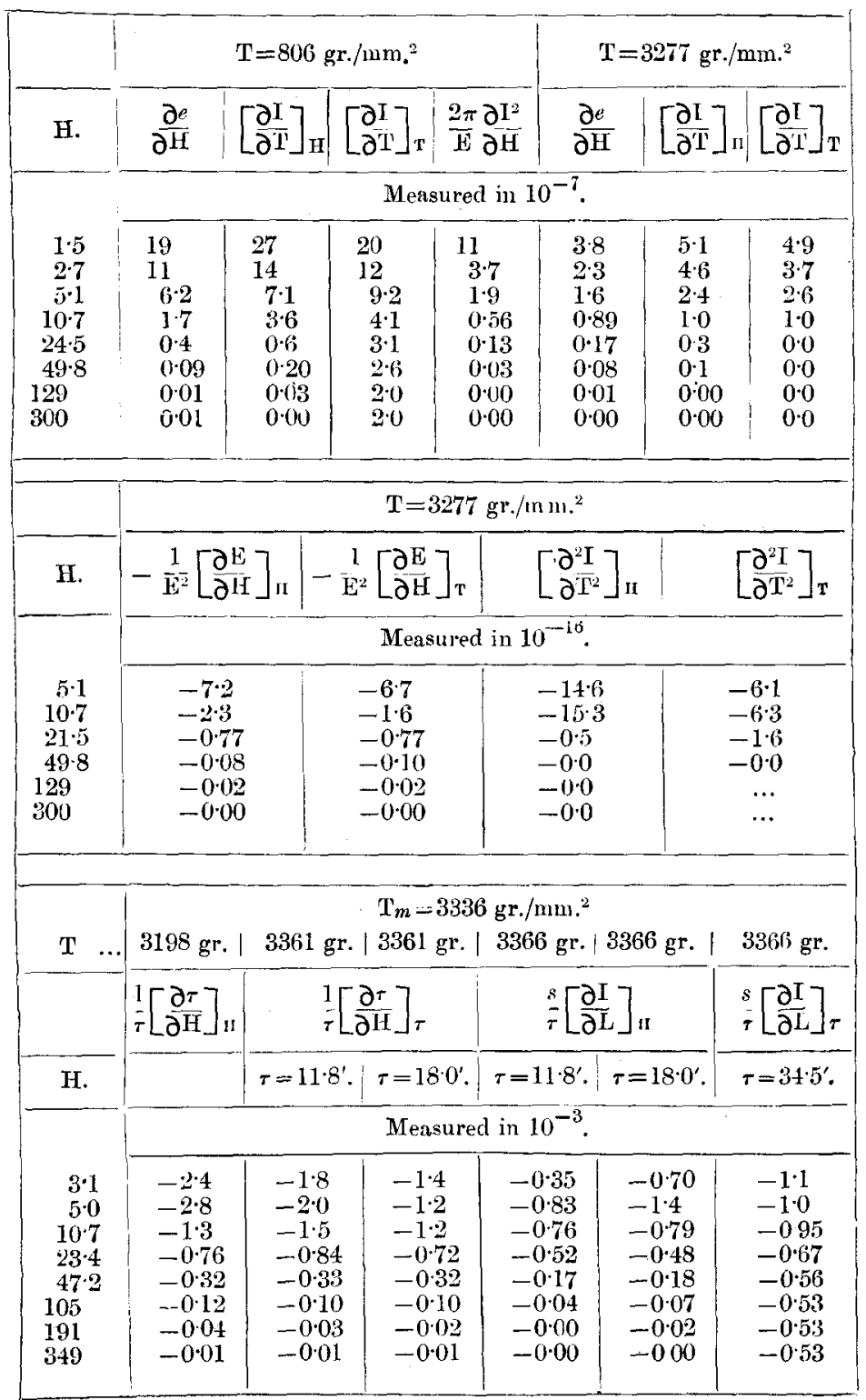

Phil. Mag. S. 6. Vol. 14. No. 79. July 1907. 
Among all the specimens tested, nickel affords the best evidence in favour of the theories above tested. The discrepancies due to the difference of the orders of applying the stress and the field, are generally small, when compared with those in the case of other specimens. The agreement is especially good in the case of tension effect, if the term $2 \pi \partial \mathrm{I}^{2}$

$\mathrm{E} \partial \mathrm{H}$ be suppressed; the differences between the values $\frac{\partial e}{\partial \mathrm{H}}$ and $\frac{\partial \mathrm{I}}{\partial \mathrm{T}}$ are of such an order of magnitude that they may be explained by the errors introduced in estimating these values from the corresponding diagrams. For the rest of the specimens, the agreement is tolerably good in many cases, except a few cases in which it completely fails. Generally speaking, the tension effect shows a better agreement between theory and experiment, if $\frac{\partial e}{\partial \mathrm{H}}$ be compared with $\left[\begin{array}{l}\partial \mathrm{I} \\ \bar{\partial} \mathrm{T}\end{array}\right]_{\mathrm{H}}$; while $\left[\frac{\partial I}{\partial T}\right]_{T}$ deduced from the $(I, H)_{T}$ curve is often of a different order of magnitude, as in the case of $28 \cdot 74$ per cent. Ni. As for the comparison between $-\frac{1}{\mathrm{E}^{2}} \frac{\partial E}{\partial H}$ and $\left[\frac{\partial^{2} \mathrm{I}}{\partial \mathrm{T}^{2}}\right]_{\mathrm{H}}$, the agreement is less remarkable, but the discrepancies may in many cases be due to the errors introduced in estimating the curvature of the curves for obtaining $\left[\frac{\partial^{2} \mathrm{I}}{\partial \mathrm{T}^{2}}\right]_{\mathrm{H}^{*}} \quad\left[\frac{\partial^{2} \mathrm{I}}{\partial \mathrm{T}^{2}}\right]_{\mathrm{T}}$ deduced from $(\mathrm{I}, \mathrm{H})_{\mathrm{T}}$ curve is often of a different order of magnitude, as in the case of Swedish iron. For torsion effect, things are much more complicated, except in the case of nickel and nickel-steels of $28 \cdot 74$ and $70 \cdot 32$ per cent. of nickel, in which the agreement is fairly good. For the last two specimens, $\frac{s}{\tau}\left[\frac{\partial \mathrm{I}}{\partial \mathrm{L}}\right]_{\mathrm{H}}$ generally agrees with $\frac{1}{\tau}\left[\frac{\partial \tau}{\partial \mathrm{H}}\right]_{\mathrm{H}}$ or $\frac{1}{\tau}\left[\frac{\partial \tau}{\partial H}\right]_{\tau}$, while in the case of 50.72 per cent. $\mathrm{Ni}$, the former is of a different sign from the latter for small twist. In all cases, the discrepancies become less in high fields.

As to the term $\frac{2 \pi}{\mathrm{E}} \frac{\mathrm{I}^{2}}{\partial \mathrm{H}_{l}}$ obtained by Gans and Sano, it may be noticed that its introduction makes the agreement between theory and experiment rather worse. The origin of this term is, however, to be traced to the fundamental assumption that at the ends of the specimen wire, the lines of induction issue 
normally from its end faces-an assumption far from being realized in our actual experiments. Hence the importance of this term must be reduced, when applied to the case usually subject to experiments.

Thus far, the agreement between theory and experiment is in general to be considered as fairly good, if we consider the difficulties encountered in measuring the minute strains caused by magnetization, and also the considerable dependence of the magnetization upon the order of magnetizing and straining. Since the theories, which are based upon quite different considerations, all agree with one another in the first important term, it may be concluded that for the first approximation, they are all verified by the present experiments. It seems, however, impossible to decide experimentally the correctness of the terms of second importance for ferromagnetic substances, in which the hysteresis effect appears in no inconsiderable amount.

In conclusion, we wish to express our best thanks to Dr. $\mathrm{S}$. Sano for useful suggestions in working out the theoretical part of the present investigation.

V. On the Forcing of Oscillations by Disturlances of Different Frequencies. By Andrew Stephenson *.

1. THE simple oscillation of a system may be influenced in two distinct ways : either by a force which is a function of the time alone, or by a force which is a function of both the time and the configuration of the system. In the former case resonance occurs only when the period of the force is equal to the free period of the system; in the latter the effect of the disturbance is cumulative when the ratio of its period to that of the system has any value within a certain range in the vicinity of $\frac{1}{2} r$, where $r$ is any integer + , the intensity being maximum when $r=1$. We now enquire as to the effect of the two different types in joint action. Will the two together under any circumstances produce and continually intensify an oscillation when each acting separately would have no appreciable result?

* Communicated by the Author.

$\dagger$ "On a class of forced oscillations," Quarterly Journal of Pure and Applied Mathematics, no. 148 (1906). The case of double frequency, $\gamma=1$, was examined by Lord Rayleigh, "On the maintenance of vibrations by forces of double frequency, and on the propagation of waves through a medium endowed with periodic structure," Phil. Mag. vol. xxiv. (188\%). 
ot Fig. 1. Swedisontron.

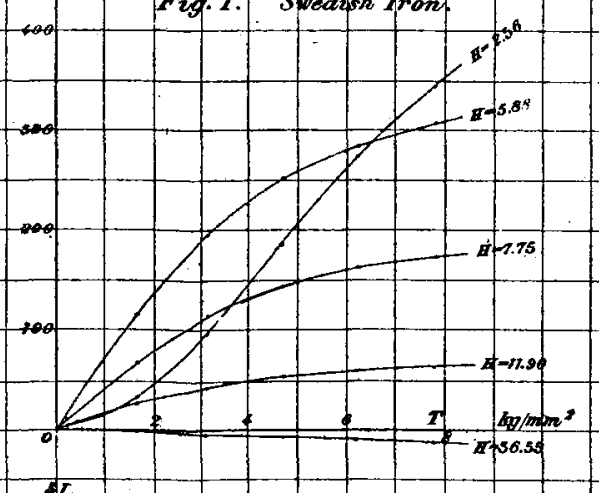

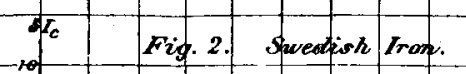

- 1 -

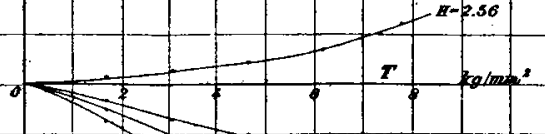

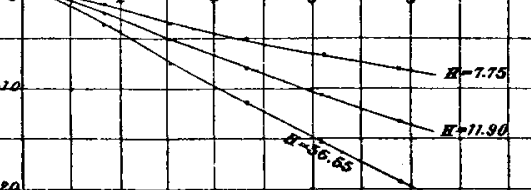

or Fig. 5. Swedksh tron

$+\infty$

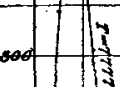

$\rightarrow 00$

.oo

(1)

00 a

Fig. 6. swedtass tran. or Fig. 3. Thangiton steel soo

200

$+$

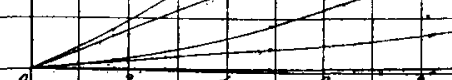

Fid. 4 . Tungsten sicet

onc

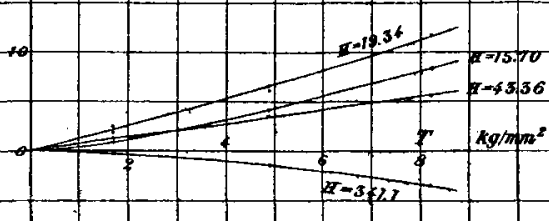

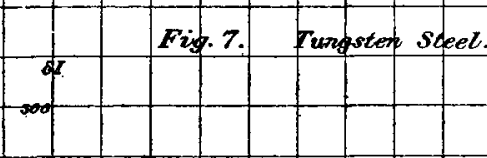
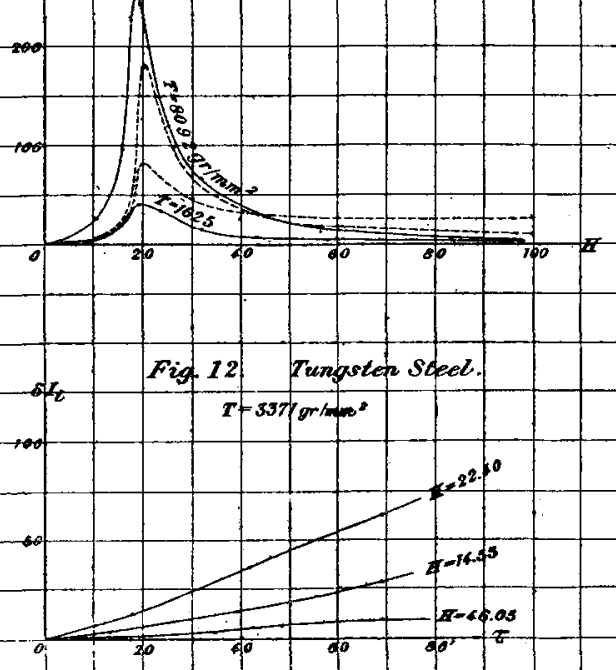

of Fig. 8. Swedish tron.

$T=152 \mathrm{~g} / \mathrm{mmm}^{2}$

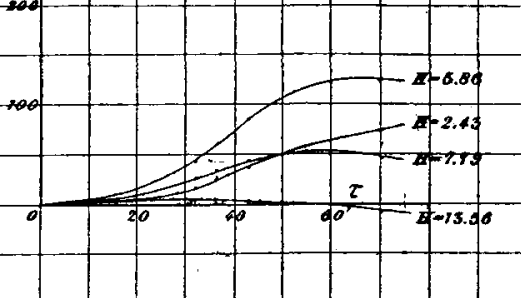

of Fig. 9. Suredian Lron

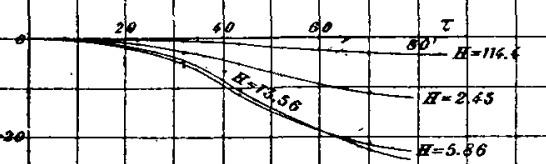

Fig. 10. Swedtis Iron

\section{of $\quad T$-3258primeses}

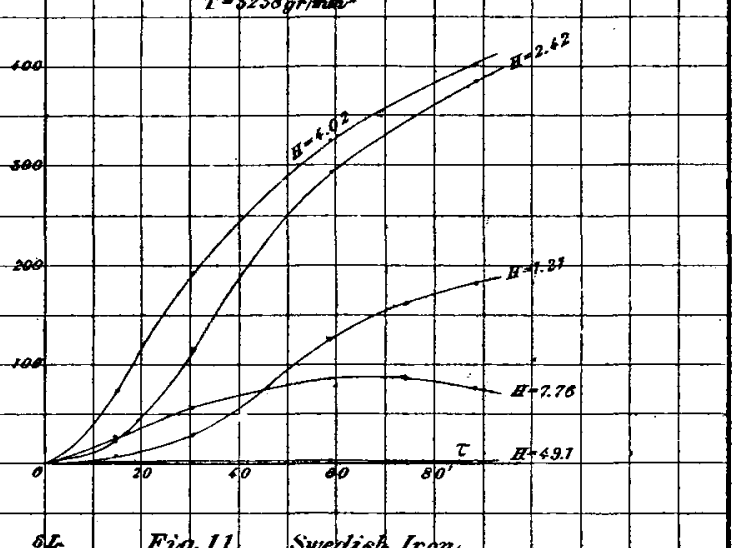

ote Fio.11 Sivederish Irom.

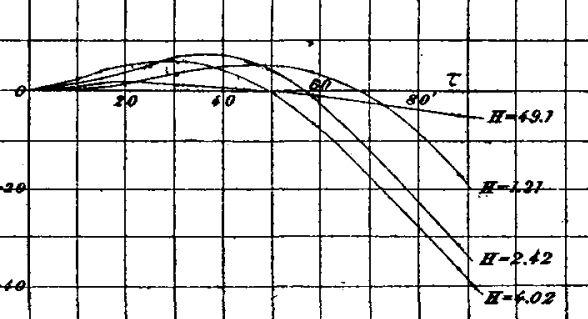




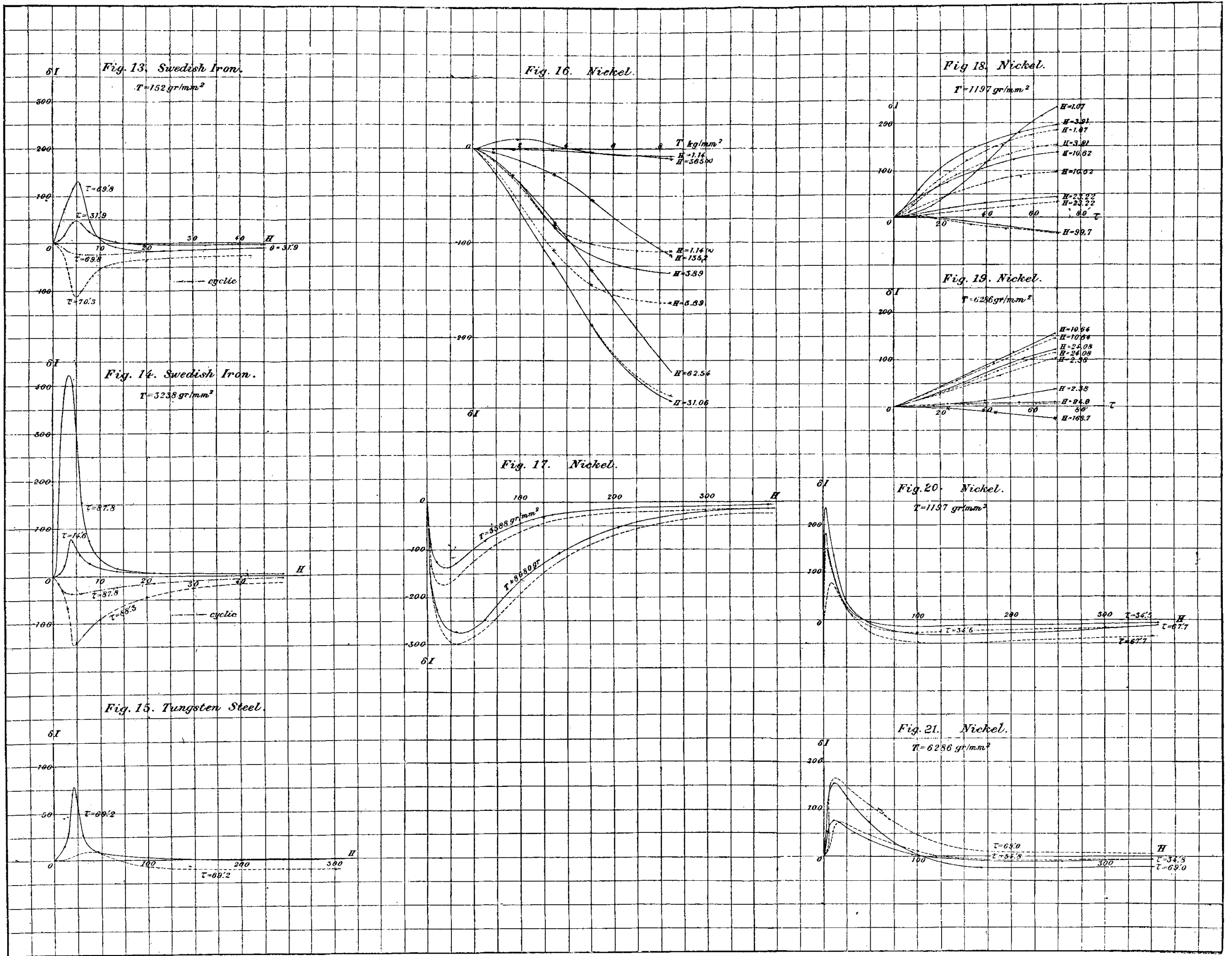




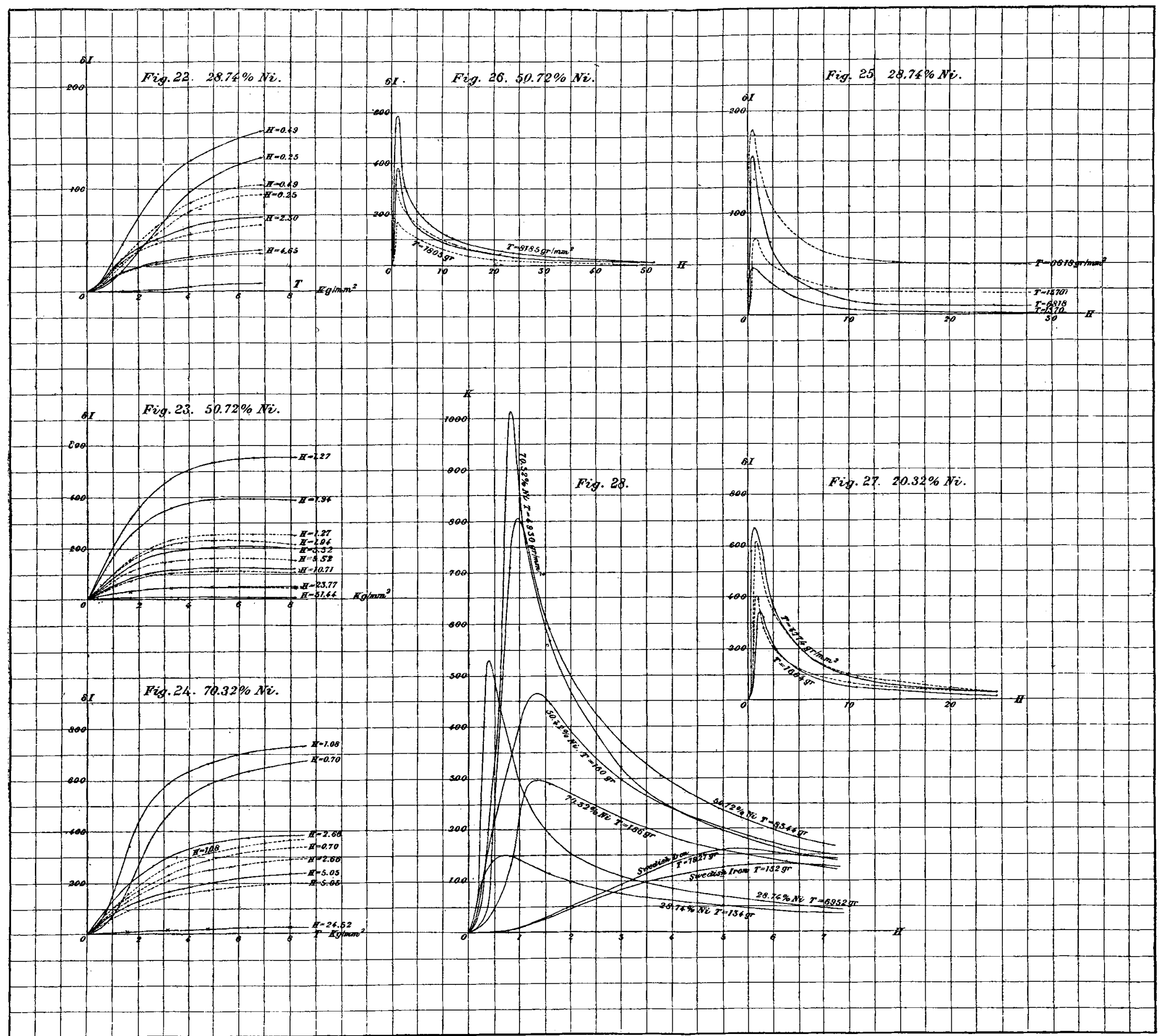




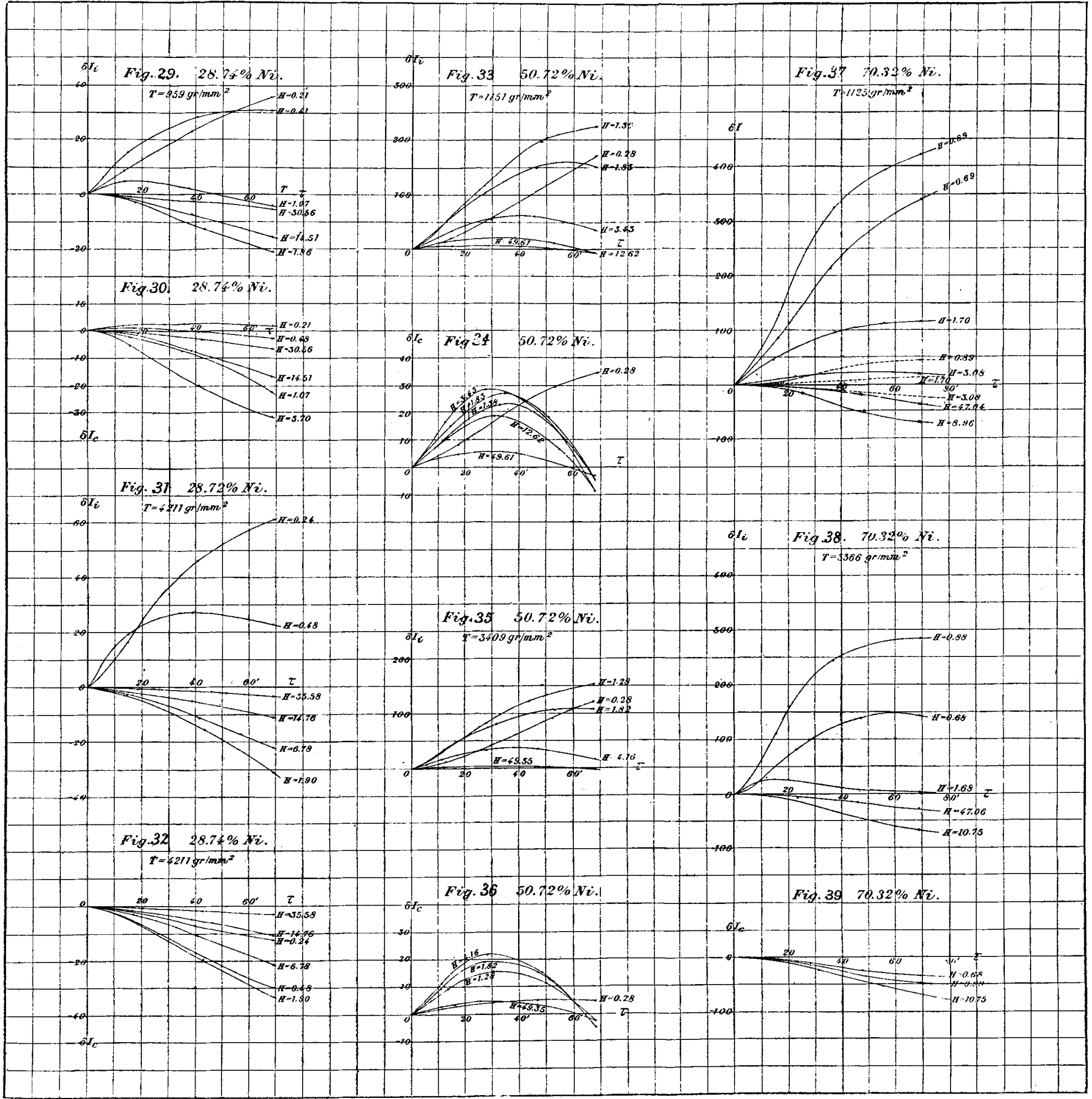




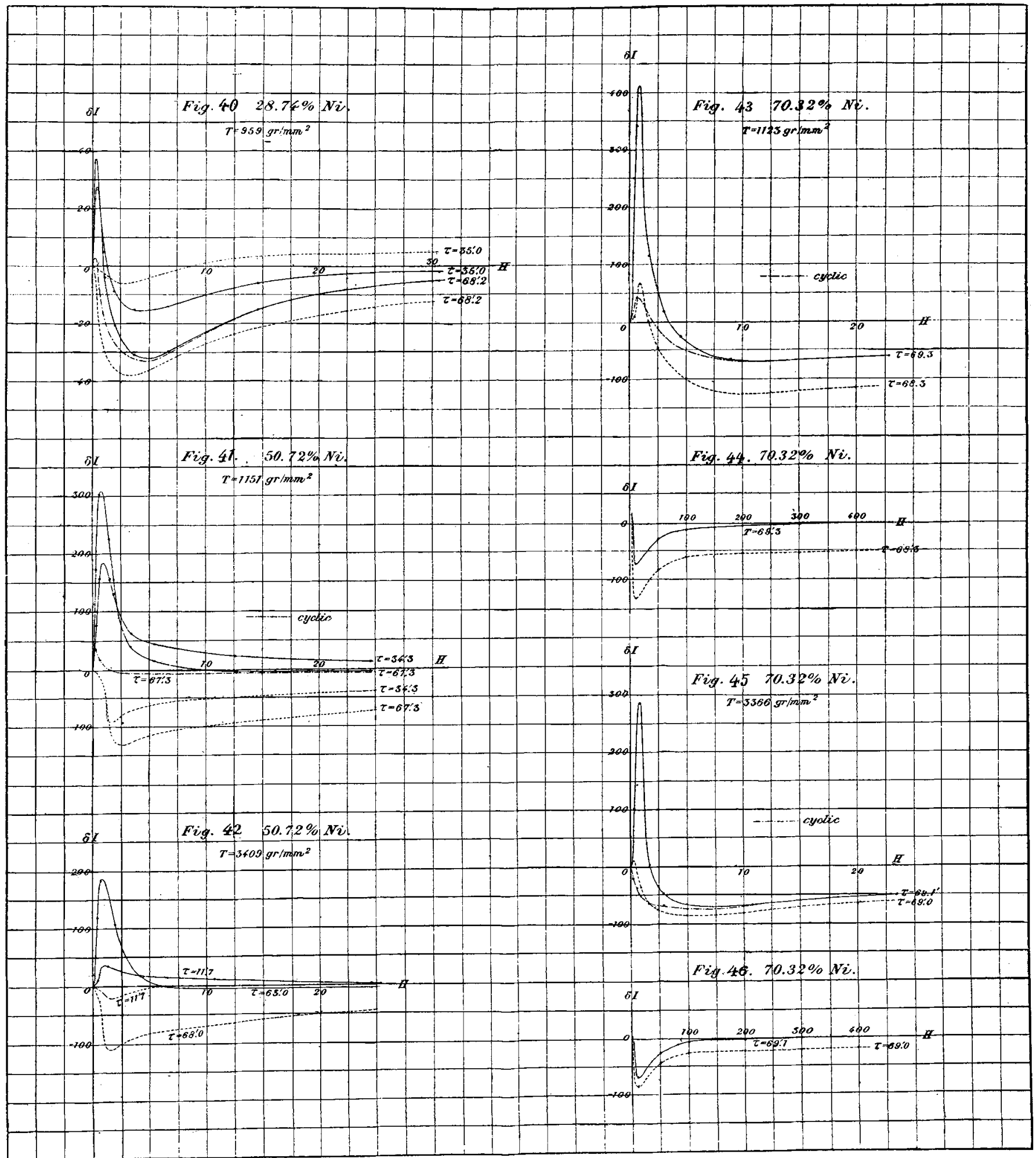

